PREPARED FOR THE U.S. DEPARTMENT OF ENERGY, UNDER CONTRACT DE-AC02-76CH03073

PPPL-3878

PPPL-3878

UC-70

Physics Basis for the Advanced Tokamak Fusion Power Plant ARIES-AT

by

S.C. Jardin, C.E. Kessel, T.K. Mau, R.L. Miller, F. Najmabadi, V.S. Chan, M.S. Chu, R. LaHaye, L.L. Lao, T.W. Petrie, P. Politzer, H.E. St. John, P. Snyder, G.M. Staebler, A.D. Turnbull, and W.P. West

October 2003
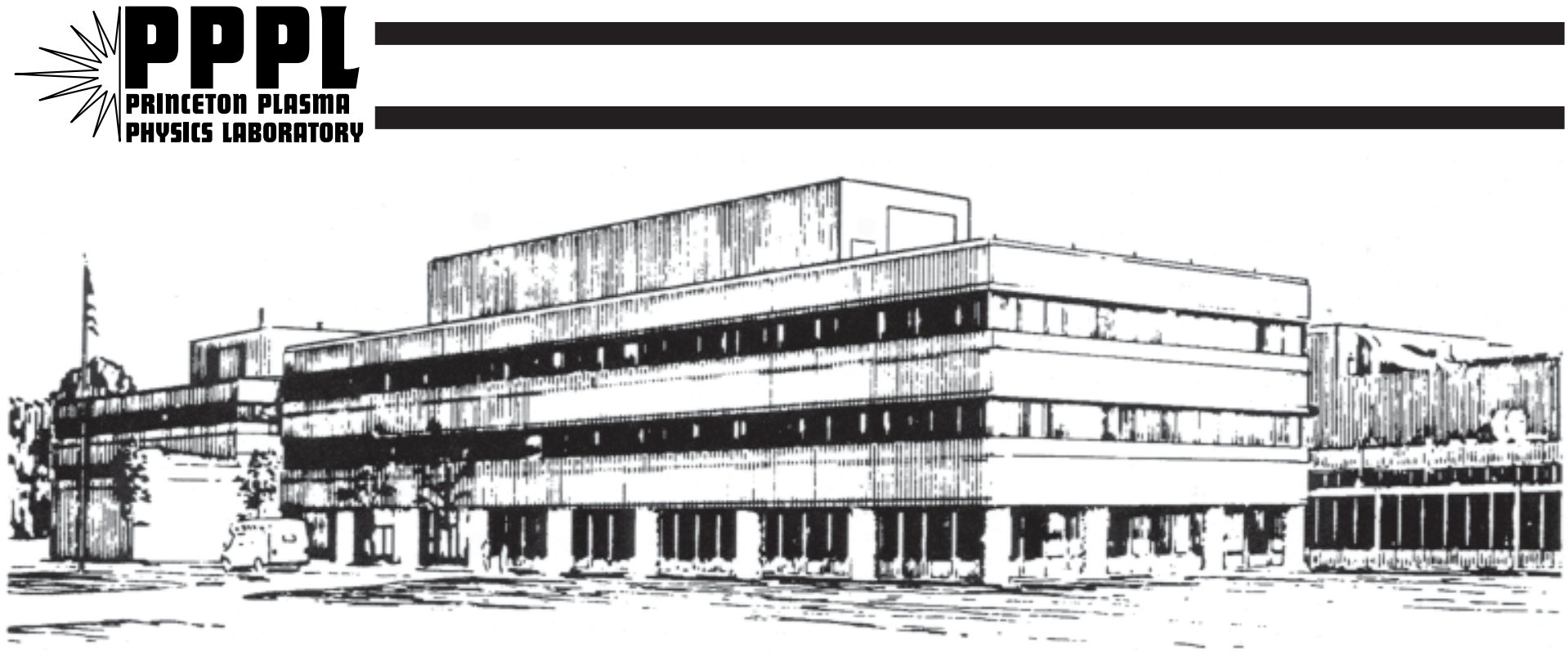

PRINCETON PLASMA PHYSICS LABORATORY PRINCETON UNIVERSITY, PRINCETON, NEW JERSEY 


\section{PPPL Reports Disclaimer}

This report was prepared as an account of work sponsored by an agency of the United States Government. Neither the United States Government nor any agency thereof, nor any of their employees, makes any warranty, express or implied, or assumes any legal liability or responsibility for the accuracy, completeness, or usefulness of any information, apparatus, product, or process disclosed, or represents that its use would not infringe privately owned rights. Reference herein to any specific commercial product, process, or service by trade name, trademark, manufacturer, or otherwise, does not necessarily constitute or imply its endorsement, recommendation, or favoring by the United States Government or any agency thereof. The views and opinions of authors expressed herein do not necessarily state or reflect those of the United States Government or any agency thereof.

\section{Availability}

This report is posted on the U.S. Department of Energy's Princeton Plasma Physics Laboratory Publications and Reports web site in Fiscal Year 2004. The home page for PPPL Reports and Publications is: http://www.pppl.gov/pub_report/

DOE and DOE Contractors can obtain copies of this report from:

U.S. Department of Energy

Office of Scientific and Technical Information

DOE Technical Information Services (DTIS)

P.O. Box 62

Oak Ridge, TN 37831

Telephone: (865) 576-8401

Fax: (865) 576-5728

Email: reports@adonis.osti.gov

This report is available to the general public from:

National Technical Information Service

U.S. Department of Commerce

5285 Port Royal Road

Springfield, VA 22161

Telephone: $1-800-553-6847$ or

(703) $605-6000$

Fax: (703) 321-8547

Internet: http://www.ntis.gov/ordering.htm 


\title{
Physics Basis for the Advanced Tokamak Fusion Power Plant ARIES-AT
}

\author{
S. C. Jardin ${ }^{1}$, C. E. Kessel ${ }^{1}$, T. K. Mau ${ }^{2}$, \\ R. L. Miller ${ }^{2}$, F. Najmabadi ${ }^{2}$, V. S. Chan ${ }^{3}$, \\ M. S. Chu ${ }^{3}$, R. LaHaye ${ }^{3}$, L. L. Lao ${ }^{3}$, T. W. Petrie ${ }^{3}$, \\ P. Politzer ${ }^{3}$, H. E. St.John ${ }^{3}$, P. Snyder ${ }^{3}$, \\ G. M. Staebler ${ }^{3}$, A. D. Turnbull ${ }^{3}$, W. P. West $^{3}$
}

\begin{abstract}
The advanced tokamak is considered as the basis for a fusion power plant. The ARIES-AT design has an aspect ratio of $A \equiv R / a=4.0$, an elongation and triangularity of $\kappa=2.20, \delta=0.90$ (evaluated at the separatrix surface), a toroidal beta of $\beta=9.1 \%$ (normalized to the vacuum toroidal field at the plasma center), which corresponds to a normalized beta of $\beta_{N} \equiv$ $100 \times \beta /\left(I_{P}(M A) / a(m) B(T)\right)=5.4$. These beta values are chosen to be $10 \%$ below the ideal MHD stability limit. The bootstrap-current fraction is $f_{B S} \equiv I_{B S} / I_{P}=0.91$. This leads to a design with total plasma current $I_{P}=12.8 \mathrm{MA}$, and toroidal field of $11.1 \mathrm{~T}$ (at the coil edge) and $5.8 \mathrm{~T}$ (at the plasma center). The major and minor radii are 5.2 and $1.3 \mathrm{~m}$. The effects of H-mode edge gradients and the stability of this configuration to non-ideal modes is analyzed. The current drive system consists of ICRF/FW for on-axis current drive and a Lower Hybrid system for off-axis. Transport projections are presented using the drift-wave based GLF23 model. The approach to power and particle exhaust using both plasma core and scrape-off-layer radiation is presented.
\end{abstract}

\footnotetext{
${ }^{1}$ Princeton Plasma Physics Laboratory, P.O. Box 451, Princeton, NJ 08543

${ }^{2}$ Fusion Energy Research Program, University of California, San Diego, 9500 Gilman Dr., La Jolla, CA 92093

${ }^{3}$ General Atomics, P.O. Box 85608, San Diego, CA 92186
} 


\begin{tabular}{|l|l|l|}
\hline Plasma Current & $I_{P}$ & $12.8 \mathrm{MA}$ \\
\hline Toroidal Field on axis & $B_{T}$ & $5.86 \mathrm{~T}$ \\
\hline Major Radius & $R$ & $5.2 \mathrm{~m}$ \\
\hline Minor Radius & $a$ & $1.3 \mathrm{~m}$ \\
\hline Elongation (x-point) & $\kappa$ & 2.20 \\
\hline Triangularity (x-point) & $\delta$ & 0.90 \\
\hline Poloidal beta & $\beta_{P}$ & 2.28 \\
\hline Toroidal beta (\%) & $\beta$ & 9.07 \\
\hline Normalized beta (Troyon) & $\beta_{N}$ & 5.4 \\
\hline on-axis safety factor & $q_{0}$ & 3.50 \\
\hline minimum safety factor & $q_{m i n}$ & 2.40 \\
\hline Bootstrap Current & $I_{B S}$ & 11.4 \\
\hline Cylindrical safety factor & $q_{*}$ & 1.85 \\
\hline Internal inductance & $\ell_{i}(3)$ & 0.29 \\
\hline peak/average density & $\frac{n_{0}}{\langle n>}$ & 1.34 \\
\hline peak/average temperature & $\frac{I_{0}}{\langle T>}$ & 1.72 \\
\hline
\end{tabular}

Table 1: ARIES AT Global Equilibrium Parameters

\section{Introduction}

This, and the companion paper on ideal-MHD-based optimizations to define a reference equilibrium [1], describe the physics basis and physics optimization studies performed for the ARIES-AT fusion power plant study. ARIES-AT is an advanced tokamak, as was ARIES-RS [2]. As such, the tokamak safety factor, or q-profile, has a "reversed shear" property where it is a local maximum on axis, decreases outward to a local minimum, and then increases to the plasma boundary. This configuration is favored for its properties that (1) it remains stable to ideal MHD ballooning and internal kink modes at very large values of the plasma $\beta \equiv 2 \mu_{0} p / B_{\text {Vac }}^{2}$, (where $B_{\text {Vac }}$ is the vacuum toroidal field evaluated at the plasma center), (2) it implies a plasma current density profile peaked off-axis that is consistent with a large bootstrapcurrent fraction $f_{B S} \sim 1$, and (3) it is consistent with favorable transport properties near and interior to the shear reversal region.

There are many similarities between this design and our earlier design study, ARIES-RS [2]. The primary difference is that we have carried the optimizations further in this design in order to produce a more aggressive, and hence more attractive configuration. This has been accomplished by considering a wider class of pressure and current profiles, by utilizing more advanced cross-sectional shaping, and by allowing the plasma region to extend further to the $\mathrm{X}$-point singularity in the confining magnetic field for the stability evaluations [1].

We have also increased both the depth and the quality of the analysis of the plasma configuration over that which was done for ARIES-RS. As described in [1], we have used a more complete prescription for the self-driven, or bootstrap current, 
that includes collisional corrections. We have considered the effects of edge pressure gradients and current density values expected for H-mode plasmas, as is described in Sec. 2.2. We have also analyzed the stability requirements for the resistive wall mode (RWM), the neoclassical tearing mode (NTM) and the edge localized modes (ELMs), as is described in the sections $2.3-2.5$.

The current drive systems for ARIES-AT are analyzed in Section 3. This includes an analysis and design specifications for the baseline current drive system of ion cyclotron resonant frequency (ICRF) and lower hybrid wave (LHW) heating and current drive. We also include analysis of backup systems, consisting of high harmonic fast wave (HHFW) and neutral beam injection (NBI). The primary function of all these systems is to drive plasma current where it is needed from plasma stability and transport considerations. However, the heating and current drive systems also are used during plasma startup, and in addition they produce plasma rotation, which affects both the MHD stability and the transport properties of the configuration.

The transport properties of the device are analyzed in Section 4. Modern transport analysis tools and the GLF23 transport model are applied to analyze the consistency of the plasma profiles with respect to evolution due to transport processes. As part of this analysis, we consider the effects of the driven rotation on plasma transport.

The power and particle exhaust properties of the plasma are analyzed in Section 5. We consider the radiative and conductive heat losses from the plasma and edge regions, and the effect of impurity doping. The engineering constraints on the maximum allowable heat fluxes on the first wall and divertor regions put severe constraints on the allowable solutions.

In Section 6 we summarize the plasma operating regime parameters in terms of a plasma operating contour (POPCON) diagram, and present an analysis of the startup requirements. The main results of this study are summarized in Section 7.

\section{Plasma Equilibrium and Stability}

The ideal MHD-based optimization procedure which led to the specification of the baseline pressure and current profiles is described in [1]. We summarize the results of that optimization in Sec. 2.1. In the following sections we consider the effects of H-mode profiles on the plasma stability (Sec. 2.2), and extend the stability analysis to include the resistive wall mode (RWM)(Sec. 2.3), the neoclassical tearing mode (NTM)(Sec. 2.4), and the edge localized modes (ELMS) (Sec. 2.5).

\subsection{Baseline Equilibrium and Profiles}

The baseline plasma profiles have zero pressure gradient and zero current density at the plasma edge. These boundary conditions (sometimes called L-mode edge) are 
conservative in that they normally lead to lower optimized beta limits than those which would be obtained by allowing the edge gradients to be nonzero. (Note that they also should be a good approximation to the post-crash phase of an H-mode ELMing discharge, see Sec. 2.5.) The effect on the plasma stability of relaxing these conditions is discussed in the next section.

The pressure profile for the reference equilibrium is given by the sum of two functions:

$$
p(\psi)=p_{0}\left[c_{1}\left(1-\hat{\psi}^{b_{1}}\right)^{a_{1}}+c_{2}\left(1-\hat{\psi}^{b_{2}}\right)^{a_{2}}\right] .
$$

The earlier design study [2], AIRES-RS, restricted the functional form of the pressure profile to a single function, which severely restricted the function space that we optimized over. The present form effectively allows separate optimizations for the core and edge regions

The result of this optimization, described in [1], are the following numerical values for the coefficients: $a_{1}=2.75, b_{1}=2.25, c_{1}=0.55$ and $a_{2}=2.00, b_{2}=$ $1.00, c_{2}=0.45, p_{0}=2.467 \times 10^{6}$. The parallel current current density profile is a self-consistent combination of the bootstrap current consistent with this pressure profile and the externally driven current profiles from fast wave and lower hybrid current drive described in the sections to follow.

In the baseline fixed-boundary equilibrium and stability studies, the plasmavacuum boundary is the $99 \%$ flux surface from the free-boundary equilibrium. Although the plasma boundary can not be described analytically, it can be parameterized in terms of a major and minor radius $R=5.2 \mathrm{~m}, a=1.30 \mathrm{~m}$, and a separatrix elongation and triangularity of $\kappa=2.20$ and $\delta=0.90$.

These parameters have been chosen to maximize the plasma $\beta$ while maintaining stability to ideal MHD modes, and to provide a high bootstrap fraction equilibrium that is compatible with the current drive systems described in Section 3. The equilibrium has a hollow current profile, with the location of the $q_{\min }$ (minimum safety factor) close to the edge.

\subsection{Effects of H-mode Like Pressure and Current Profiles}

The equilibrium and stability analysis described in Ref. [1] and in Sec. 2.1 was based on "standard" pressure and current profile functions that go smoothly to zero at the plasma edge, with zero pressure derivative there. This class of profiles, which have traditionally been used in stability studies, are typical of L-mode plasmas and of post-ELM H-mode plasmas. Because ARIES-AT requires the energy confinement times typical of an H-mode, we have extended the stability analysis to examine the effects of the edge gradients typical of pre-ELM H-mode plasmas. 


\begin{tabular}{|l|l|l|l|}
\hline & $\begin{array}{l}\text { Marginal Wall } \\
n=1\end{array}$ & $\begin{array}{l}\text { Marginal Wall } \\
n=2\end{array}$ & $\begin{array}{l}\text { Marginal Wall } \\
n=3\end{array}$ \\
\hline $\begin{array}{l}\text { L-Mode Limiter } \\
\beta_{N}=5.6\end{array}$ & 1.525 & 1.450 & 1.350 \\
\hline $\begin{array}{l}\text { L-Mode Divertor } \\
\beta_{N}=5.6\end{array}$ & 1.600 & 1.475 & 1.425 \\
\hline $\begin{array}{l}\text { H-Mode Divertor } \\
\beta_{N}=5.8\end{array}$ & 1.550 & 1.475 & 1.450 \\
\hline $\begin{array}{l}\text { H-Mode Divertor } \\
\beta_{N}=5.9\end{array}$ & 1.575 & 1.450 & 1.400 \\
\hline
\end{tabular}

Table 2: Comparison of the critical wall location for stabilization against the $n=$ 1,2,3 ideal modes for the reference and H-mode like equilibria.

\subsubsection{H-Mode Equilibrium}

The primary difference between the plasma profiles defined in Sec. 2.1 and the HMode profiles defined here are the values of the pressure gradient and current density near the plasma edge. Because the stability of the H-mode profiles depends sensitively on the interplay of the strong shaping near the edge of a divertor plasma and the steep gradients there, we have recomputed the free-boundary diverted equilibrium with (H-mode-like) pressure and current profiles using the EFIT code. These profiles were based on the reference equilibrium described in the previous section. This new equilibrium [3] with non-zero edge pressure gradients forms the basis for the stability analysis in the remainder of this section.

In this procedure, the pressure and the current profiles from the reference (Lmode like) equilibrium are first fit to a set of polynomial basis functions. H-mode like equilibria are then generated by perturbing the outer region of the reference equilibrium obtained using the fitted profiles while keeping the core as similar to the reference case as possible. The edge pressure gradient and current density are allowed to be finite. This is illustrated in Figure 1, where the two free pressure and poloidal current functions, $P^{\prime}(\psi)$ and $F F^{\prime}(\psi)$, the safety factor $q(\psi)$, and the flux surfaces for the reference and H-mode like equilibria are compared. This particular H-mode like equilibrium has $P^{\prime}(1) \sim 0.2 P^{\prime}(0),\left\langle J_{T}\right\rangle(1) \sim 0.38 J_{\text {ave }}, \beta_{T} \sim 9.7 \%$, and $\beta_{N} \sim 5.9$. Because of the finite edge pressure gradient, the new (H-mode like) case has a slightly higher $\beta$ value.

\subsubsection{Effect on Ballooning and Kink Stability}

Ideal stability analyses indicates that stability against the low $n=1,2,3$ modes are relatively insensitive to the presence of the X-point and the broader $\mathrm{H}$-mode like pressure and current profiles width. The locations of the conducting wall required for stabilization against these modes remain similar. This is summarized in Table 2. 

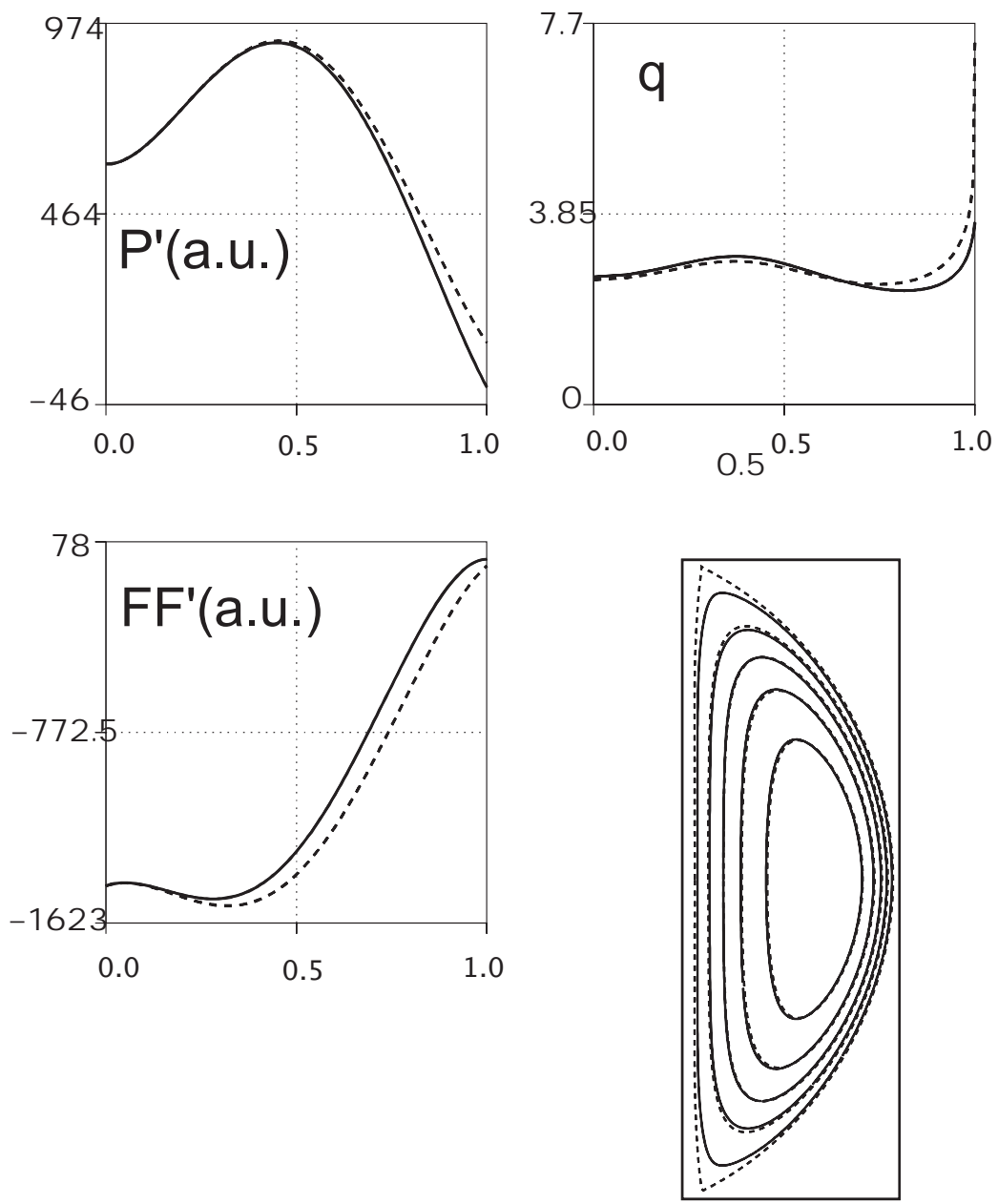

Figure 1: Comparison of the pressure and poloidal current functions $p^{\prime}(\psi)$ and $F F^{\prime}(\psi)$, the safety factor profile $q(\psi)$, and the flux surfaces for a H-mode like equilibrium (dashed curves) against those for the reference (L-mode like) base equilibrium (solid curves). The L-mode reference case has a limiter shape with no X-point on the boundary, whereas the H-mode like case has a divertor shape with $2 \mathrm{X}$-points on the boundary. 
Stability against the high- $n$ ideal ballooning modes, which are limiting the reference limiter equilibrium, are actually improved by the H-mode like profiles, even though the pressure gradients and $\beta$ value are higher. With sufficiently high edge pressure gradient and bootstrap current, the H-mode like equilibria have second stability access for ballooning modes over essentially the entire plasma volume. This is illustrated in Figure 2 for the H-mode like equilibrium shown in Figure 1.
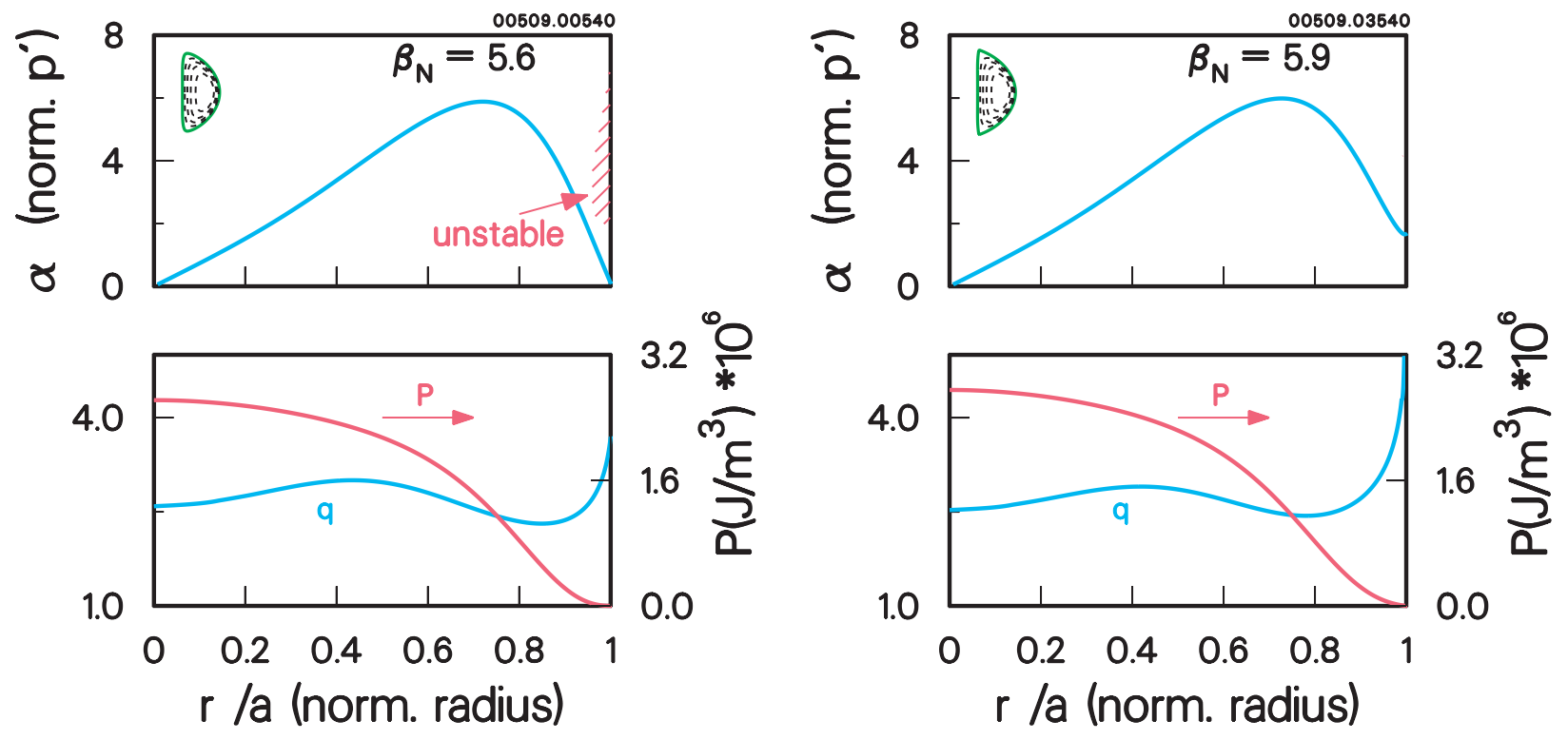

Figure 2: Comparison of Ballooning stability for the reference (left) and H-mode like (right) equilibria.

\subsection{Stability to the Resistive Wall Mode (RWM) and its Stabilization by Plasma Rotation}

The ARIES-AT equilibria [3] was tested for its stability to the Resistive Wall Mode (RWM) and stabilization of the RWM by plasma rotation was evaluated by using the MARS stability code [5]. MARS is an eigenvalue code, which solves for the full MHD perturbation equations with the MHD mode frequency as the eigenvalue and the perturbed MHD quantities (perturbed plasma displacement, magnetic field and pressure) as eigenfunctions. One of the most important input quantities in the present study is the plasma rotation profile. For simplicity, we assume that the plasma has a constant(rigid) rotation frequency profile across its cross-section. The model for the damping of the toroidal momentum used is the sound wave damping model. In this model, there is a force which damps the perturbed toroidal motion of the mode according to the formula 


$$
F_{S D}=-\kappa_{\|} \sqrt{\pi}\left|k_{\|} v_{t h_{i}}\right| \rho \vec{v} \cdot \hat{b} \hat{b} .
$$

Here $\kappa_{\|}$is a numerical coefficient chosen to model the ion Landau damping process, $k_{\|}$is the parallel wave number $(m-n q) / R, v_{t h_{i}}$ is the ion thermal velocity, $\rho$ is the mass density, $\vec{v}$ is the perturbed plasma velocity and $\hat{b}$ is the unit vector of the equilibrium magnetic field. In this study, the value of $k_{\|}$is chosen to be 0.89 , which has been found to best fit the DIIID experimental data [4]. This value is half of the value of 1.77 predicted by the theoretical ion sound wave damping model.

\subsubsection{RWM with Toroidal Mode Number $n=1$}

The critical rotation frequency for stabilization of the RWM in the H-mode equilibria [3] is found to be between 0.07 to 0.08 of the Alfven transit frequency. No stability window is found for rotation frequency below 0.07. The stability window for the location of the resistive wall is between 1.3 and 1.45 of the plasma radius when the plasma rotation frequency is at 0.08 of Alfven frequency. This window widens to between 1.075 to 1.45 of the plasma radius when the rotation frequency is increased to 0.09 of the Alfven frequency. Here, the Alfven frequency is defined as

$$
f_{a v}=\frac{v_{A}}{R}
$$

where $R$ is the plasma center and $v_{A}$ is defined as

$$
v_{A}=\frac{B_{v a c}}{\sqrt{\mu_{0} \rho_{0}}}
$$

- A uniform density 50-50\% $D-T$ plasma with electron density $2.2 \times 10^{20} / \mathrm{m}^{3}$ is used and the value of $f_{a v}$ is computed to be $1.06 \times 10^{6} / \mathrm{sec}$. The resistive wall time has been taken to be $5000 \tau_{a}$ or 5 msec. When the rotation frequency is increased beyond .09 of the Alfven frequency, the growth rate normalized to the wall time is reduced below 0.1. It would then have a time scale longer than $50 \mathrm{msec}$, or a frequency less than $20 \mathrm{~Hz}$.

These results are illustrated in the following figures. Shown in Figure 3 is the computed mode structure from MARS. It shows the poloidal Fourier harmonics of the perturbed radial magnetic field of an unstable RWM stabilized by plasma rotation at $8 \%$ of the Alfven frequency, with a growth rate of $\gamma \tau_{W}=0.28$. The location of the external resistive wall is at 1.35 times the plasma radius. It is observed that this mode has a global structure. Shown in Figure 4 is the growth rate of the RWM as a function of the location of the resistive wall. Each curve has a different plasma rotation frequency relative to the resistive wall. The same equilibrium is used here as in Figure 3. 


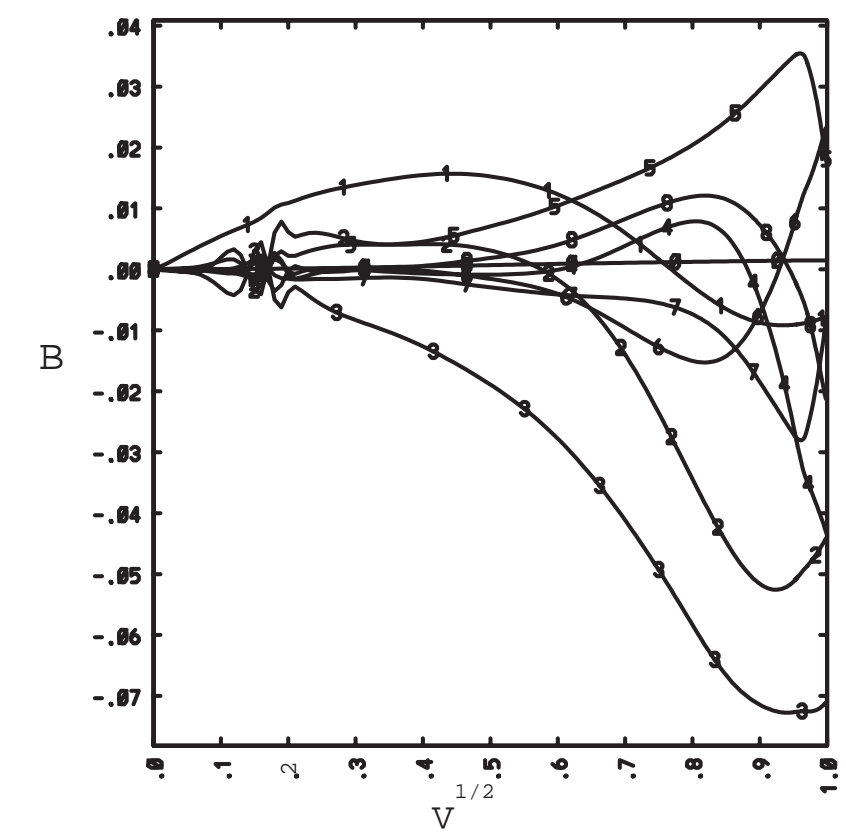

Figure 3: Amplitudes of the poloidal harmonics of the perturbed magnetic field $\delta B_{\psi}$ of a stabilized RWM. Here $\delta B_{\psi}$ is plotted as a function of $\sqrt{V}$ for equilibria [3]for a uniformly rotating plasma with $\Omega=.08$ measured in units of Alfven toroidal transit frequency and with $r_{w}=1.35 r_{p}$ and toroidal mode number $n=1$

\subsubsection{RWM with Toroidal Mode Number $n=2$}

A similar study has also been performed for the $n=2$ mode. It is found that the rotation required for the $n=2$ mode is substantially reduced from that for the $n=1$ mode. This reduction factor is close to 2, as shown in Figure 5. In this figure growth rates of the resistive wall mode with the plasma rotating at different rotation frequencies are plotted as a function of the location of the resistive wall. Note that there is an opening up of the stability window first at $r_{w}=1.5$ at around rotation frequency of 0.03 of the Alfven frequency. When the rotation frequency is increased to 0.05 of the Alfven transit frequency, this window widens to between $r_{w}=1.32$ and $r_{w}=1.5$.

The reason for this reduction is the increased sound wave damping due to the increased number of resonant flux surfaces. The corresponding mode structure of the $n=2$ RWM is shown in Figure 6. The mode structure is still quite global. 


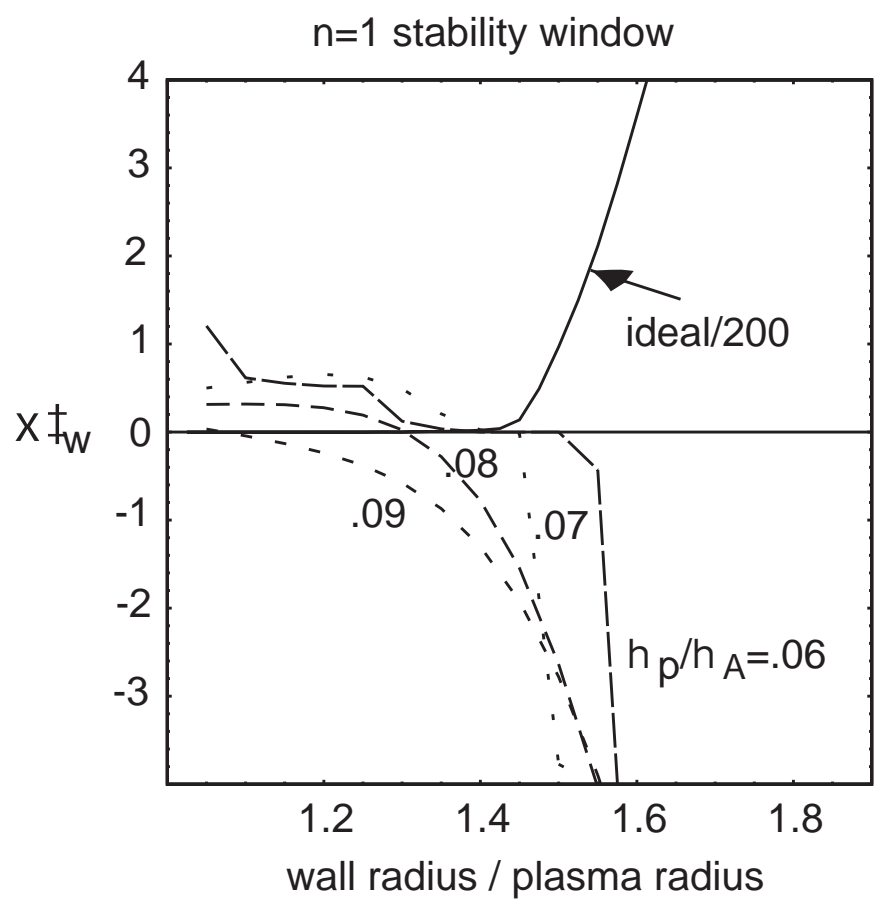

Figure 4: Stability window in $r_{w}$ for the $n=1$ RWM for equilibrium [3]. Plotted are growth rates of the ideal and resistive wall modes vs. $r_{w}$. The curve labeled ideal is the growth rate of the ideal external kink with the scales multiplied by 200 . Other curves are labeled by the rotation frequency of the plasma with respect to the resistive wall measured in units of the toroidal Alfven transit frequency. The growth rates of the resistive wall modes are multiplied by $\tau_{w}$.

\subsubsection{Stability to the RWM through use of Intelligent Shell Feedback}

It was found in Sections 2.3.1 and 2.3.2 that the rotation frequency required for the stabilization of the RWM is over a few percent of the Alfven wave transit frequency. As is shown in Sections 4.2 and 4.3, this amount of rotation would be difficult to maintain for a net-power producing tokamak such as ARIES-AT. Therefore, the alternative approach of active feedback stabilization of the RWM has also been studied. In the intelligent shell feedback scheme, external coil currents are utilized to make the resistive wall appear almost ideally conductive to the plasma. This approach has been formulated for a finite $\beta$ plasma in toroidal geometry by extending the ideal MHD energy principle, and implemented numerically by coupling GATO [6] with VACUUM [7]. Application of this formulation to the ARIES-AT 


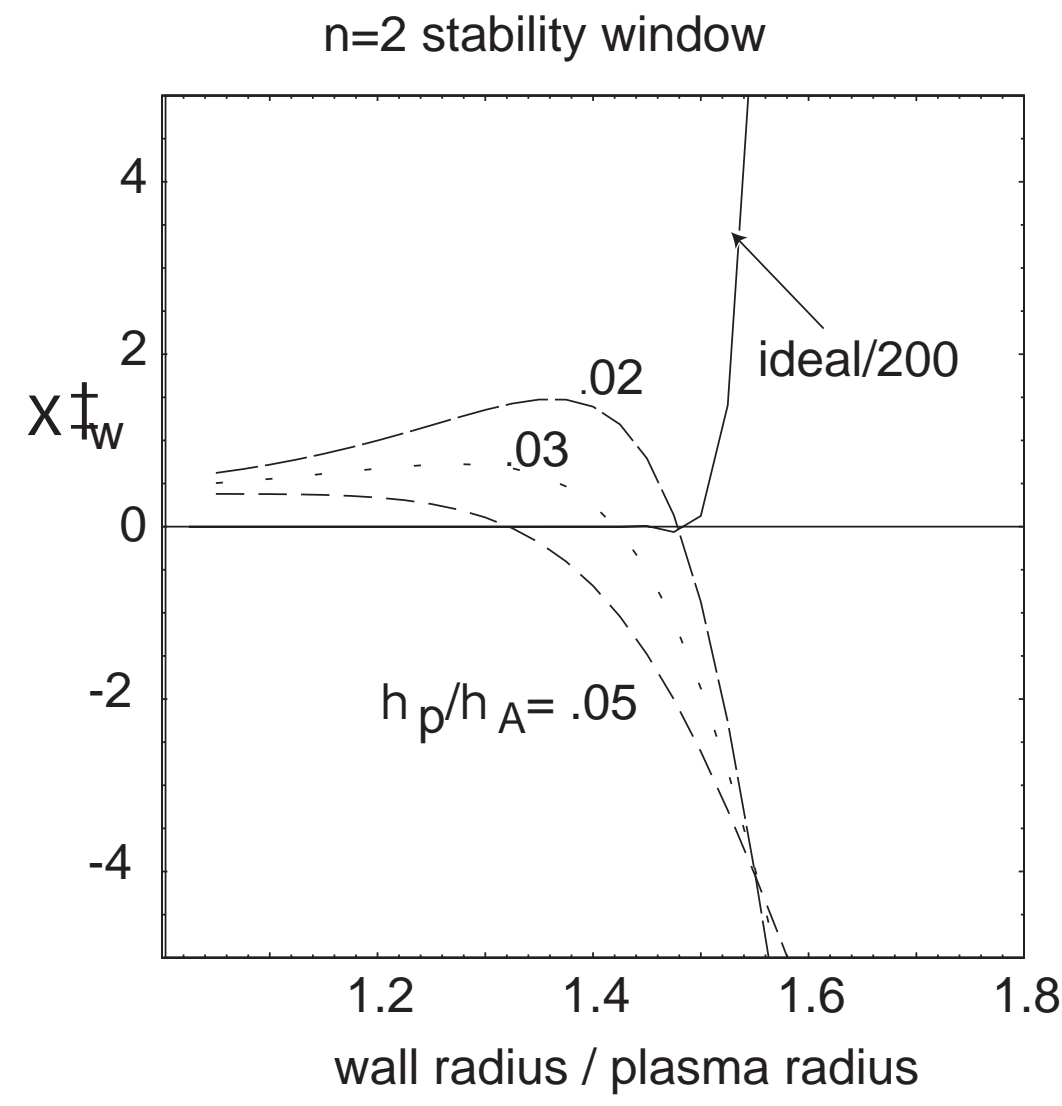

Figure 5: Stability window in $r_{w}$ for the $n=2$ resistive wall mode for equilibrium [3]. Plotted are growth rates of the ideal and resistive wall modes vs. $r_{w}$. The curve labeled ideal is the growth rate of the ideal external kink with the scales multiplied by 200. Other curves are labeled by the rotation frequency of the plasma with respect to the resistive wall measured in units of the toroidal Alfven transit frequency. The growth rates of the RWMs are multiplied by $\tau_{w}$.

geometry[3], and employing "flux conserving intelligent coils" located on the resistive wall, indicated that the resistive wall can be made to be $90 \%$ effective to $n=1$ RWM by covering the resistive wall with 7 segments of poloidal coils of equal poloidal coverage. Here, the effectiveness of the coil arrangement is defined as

$$
E f f=\frac{\delta W-\delta W_{\eta_{W}=\infty}}{\delta W_{\eta_{W}=0}-\delta W_{\eta_{W}=\infty}} .
$$

The baseline case is stable with the wall ideally conducting, $\left(\delta W_{\eta_{W}=0} \geq 0\right)$, and $\delta W_{\eta_{W}=\infty}$ is -0.35 . The value of $\delta W_{\eta_{W}=0}$ of 0.04 was inferred by increasing the value of $\beta$ to reach marginal stability with the ideal wall. Therefore the reference design case has a $\beta$ value at $90 \%$ of the ideal wall limit. These results imply that the 


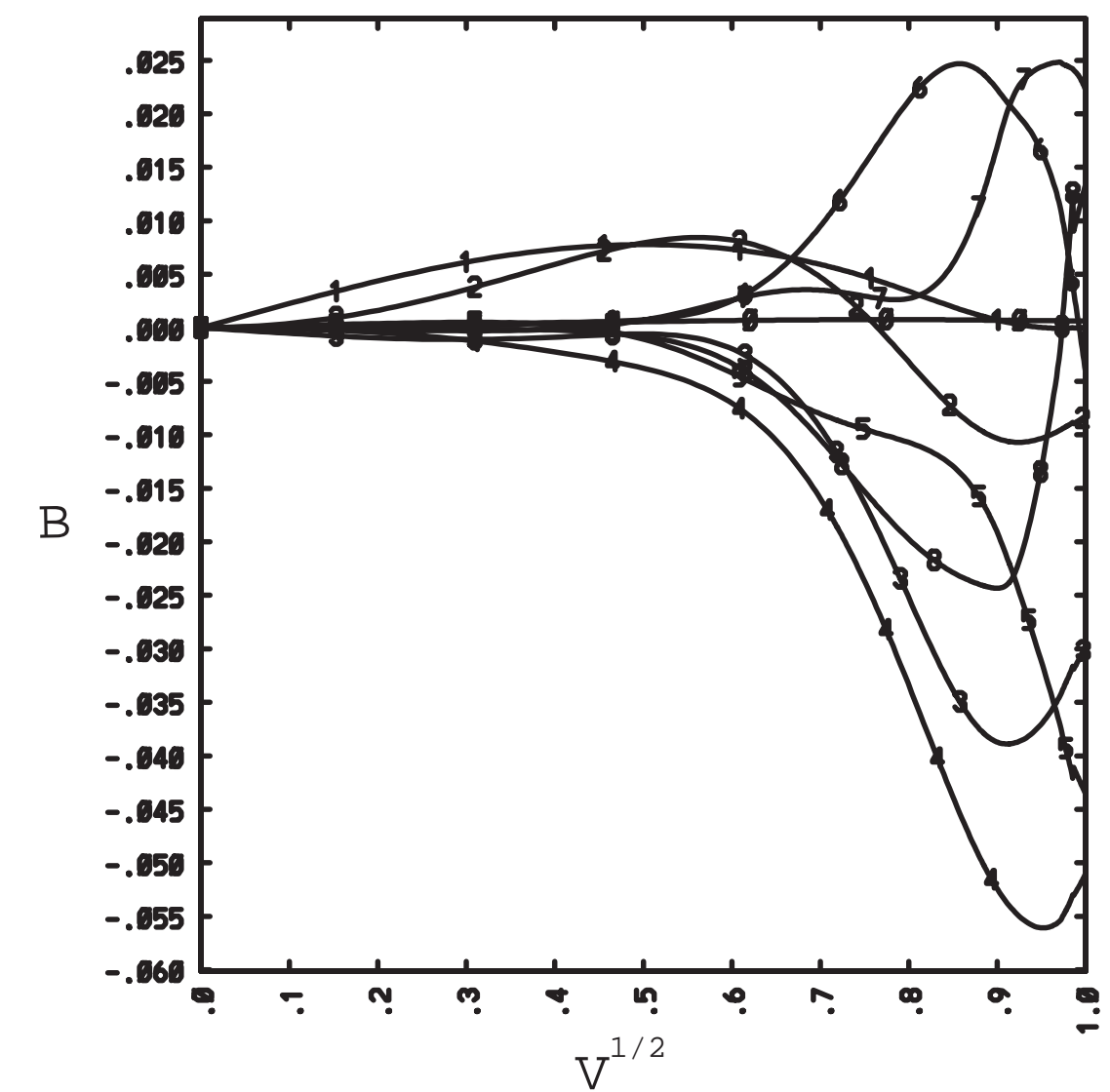

Figure 6: Amplitudes of the poloidal harmonics of the perturbed magnetic field $\delta B_{\psi}$ of a stabilized RWM with toroidal mode number $n=2$. Here $\delta B_{\psi}$ is plotted as a function of $\sqrt{V}$ for equilibrium [3] for a uniformly rotating plasma with $\Omega=.08$ measured in units of Alfven toroidal transit frequency and with $r_{w}=1.35 r_{p}$. 
reference baseline case is stable to the $n=1$ RWM with smart shell feedback logic. This is implemented in the ARIES-AT design by covering the resistive shell with 7 segments of poloidal coils of equal poloidal angular extent.

\subsubsection{Evaluation of Resistive Wall Mode Control Coil Requirements for ARIES-AT}

The $n=1$ resistive wall mode (RWM) is an ideal kink which occurs when beta exceeds the no-wall beta limit but is less than the ideal-wall beta limit. Since any real wall is not ideal but resistive with an effective wall time $\tau_{W}$, the RWM can grow but is slowed to a growth rate $\gamma \leq \tau_{W}^{-1}$ and to a rotation frequency $\omega \leq \tau_{W}^{-1}$ by the resistive wall. The RWM manifests itself as an $n=1$ radial field $\tilde{B}_{R}$ coming through the wall. The slowing down of the mode growth makes stabilization tractable by feedback control with an external (to the vacuum vessel) coil which opposes the change in $\tilde{B}_{R}$ at the wall, i.e., acts to make the wall behave as a perfect conductor $[8,9]$. Experiments in the tokamaks HBTX at Columbia University and DIII-D at General Atomics are presently implementing such a feedback control to provide an experimental demonstration of RWM feedback.

A conceptual feedback coil for ARIES-AT is shown in Figure 7. It is based on the "C-Coil" in DIII-D [10]. Assuming the copper vertical stabilizing shells act to inhibit $n=1$ flux penetration, the feedback coil must oppose any $n=1$ flux leakage through the vacuum vessel at the outboard midplane. An array of integrated "saddle loop" sensors will be placed on the inside vessel wall to detect the mode and act in the feedback loop. The coil top and bottom are at $Z_{C}= \pm 1.45 \mathrm{~m}, R_{C}=7.5 \mathrm{~m}$, to subtend a poloidal angle of $60^{\circ}$ from the plasma axis; this is about $1 / 2$ a poloidal wavelength for the dominant $m=3$ component. A set of eight "windowpane" coils, subtending $45^{\circ}$ wide each toroidally, covers the torus and fits into the $N=16$ symmetry of the TF-coil. These would be hooked up into four pair with $180^{\circ}$ opposite coils connected in anti-series for $n$ odd with $n=1$ dominant. Thus four independent power supplies are required. The coils must be designed to avoid port obstructions, or alternatively a configuration of sixteen- $22.5^{\circ}$ wide coils making up an equivalent ensemble might be desirable.

The present design only explicitly considers modes with $n=1$, since RWMs with $n>1$ have not been experimentally observed. (However, we note that this could be due to the fact that at present, in most of the experimental discharges, the achieved $\beta_{N}$ is relatively low $(\leq 3-4)$ and therefore more stable to the $\mathrm{n}=2$ external kink mode. The $n=2$ mode is predicted to be unstable at higher values of $\beta_{N}$ with larger edge pressure gradients.) The feedback system to be described should also be effective for $n=2$ modes, but this issue needs to be addressed in more depth in future investigations.

In DIII-D, modes are detectable at the level $\left|\tilde{B}_{R}\right| \approx 1 G$ in a discharge with axial toroidal field $B_{T 0}$ of $2 T$ or about $5 \times 10^{-5}$ of $B_{T 0}$. The C-Coil in DIII-D can produce field at the sensor up to 50 times this. Thus each (of four pair) RWM coils 


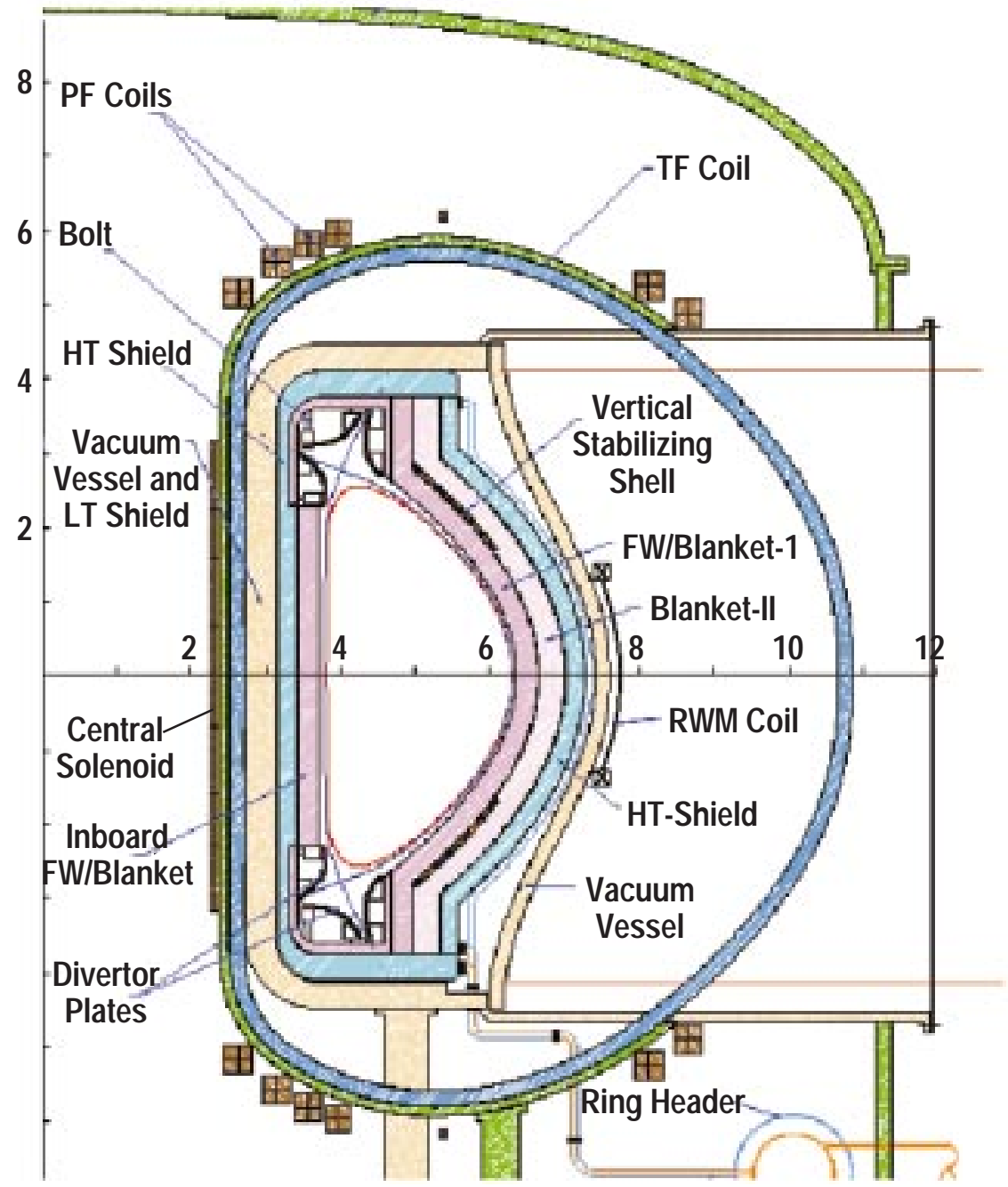

Figure 7: Cross-section of ARIES-AT power core configuration showing the proposed RWM control coil. 


\begin{tabular}{|l|l|}
\hline$\#$ coils & $16^{\circ}-22.5^{\circ}$ wide in $\phi, 60^{\circ}$ wide in $\theta$, outboard \\
\hline$N$ turns & 4 \\
\hline$R_{C}, Z_{C}$ & $7.50 \mathrm{~m}, \pm 1.45 \mathrm{~m}$ \\
\hline$\tilde{B}_{R}$ at vessel & $150 \mathrm{G}$ \\
\hline$I_{C}$ & $50 \mathrm{kA}$-turns \\
\hline$\omega \tau_{W}$ & $\leq 3$ so $f \leq 25 \mathrm{~Hz}$ for $\left.\tau_{W} \approx 20 \mathrm{msec}\right)$ \\
\hline Resistance $R$ & $17 m \Omega($ each of four sets, $2.6 \mathrm{~cm} \mathrm{OD} \mathrm{Cu}$ turns) \\
\hline$L_{C}=N^{2} \mu_{0} R_{C}$ & $9 N^{2} \mu H$ \\
\hline$I_{C} / N$ & $13 \mathrm{kA}$ from each supply (of four) \\
\hline$V_{C}=3 N \mu_{0} R_{C} I_{C} / \tau_{W}$ & $300 \mathrm{volts}$ from each supply (of four) \\
\hline$\left(I_{C} / N\right) V_{C} / 2$ & $2 \mathrm{MW}$ from each supply (of four), reactive power \\
\hline$\left(I_{C} / N\right)^{2} R / 2$ & $1.5 \mathrm{MW}$ (each of four), dissipated power \\
\hline$\delta_{T} / \delta_{t}$ & $1.5^{\circ} \mathrm{C} /$ sec (no cooling assumed) \\
\hline
\end{tabular}

Table 3: RWM Feedback Coil Requirements

in ARIES-AT must make about $50 \times 5 \times 10^{-5} \times 5.9 \times 10^{4} G \approx 150 G$ at the outboard midplane vacuum vessel. The necessary current is about $I_{C} \approx \pi Z_{C}\left|\tilde{B}_{R}\right| / \mu_{0}=50$ kA-turns.

The number of turns and bandwidth of the four independent power supplies depends on the necessary frequency $\omega$ which in turn depends on the wall time $\tau_{W}$. Neglecting the radially thick Li-Pb blankets of relatively high resistivity $(1.2 \mu \Omega-m)$ which have $\mathrm{Li}-\mathrm{Pb}$ in $\mathrm{SiC}$ channels and also neglecting the copper vertical stabilizing shells, (The copper vertical stabilization shells are expected to be able to further slow down the growth rate of the RWM. This allows a longer response time of the external feedback circuit. The design listed in Table 3 can be considered as conservative estimates.) One assumes the same geometry and material vacuum vessel as DIII-D with $\tau_{W}$ scaled up by size squared from 5 msec in DIII-D to about 20 msec in ARIES-AT. Requiring a linear amplifier with a voltage capable of driving $\pm I_{C} / N$ up to $\omega \tau_{W} \approx 3(\omega / 2 \pi \approx 25 H z)$ sets $V_{C} \approx \omega L\left(I_{C} / N\right)$ where $L$ is the inductance of one pair in anti-series, $N$ is the number of turns, we neglect the coil resistance and any cabling impedance, and we neglect any current induced in the vessel. Now $L \approx N^{2} \mu_{0} R_{C}$ per pair and $V_{C} \approx 3 N \mu_{0} R_{C} I_{C} / \tau_{W} \approx 300 \mathrm{~V}$ for $N=4$ with $I_{C} / N \approx 13 k A$ or $0.5 V_{C}\left(I_{C} / N\right) \approx 2 M W$ each of four supplies (linear amplifiers). Careful design to minimize cabling impedance and coil resistance is assumed here. Such linear amplifiers are of order of what is operational on DIIID. We note that with the present coil design of using eight coils connected into four pairs, the stabilization of the $n=2$ RWM can also be easily accommodated. Requirements for the coil and the power supplies/linear amplifiers are summarized in Table 3. A further requirement on the linear amplifiers is negligible phase shift up to $\omega \tau_{W} \approx 10$ so that the closed loop feedback system does not go unstable.

An additional use of the RWM Coil would be for error field correction, the 
original purpose of the C-Coil in DIII-D. Asymmetries in winding and positioning $\mathrm{PF}$ and TF coils produce resonant $n=1$ magnetic field non-axisymmetry which can slow rotation, destabilize $n=1$ RWMs, or lead to locked modes. As in the DIII-D C-Coil [11], the proposed RWM Coil can be operated in a duplex fashion to both correct "dc" error field and to feedback stabilize "ac" RWMs.

\subsection{Neoclassical Tearing Mode Control Requirements}

The neoclassical tearing mode (NTM) island results when: (1) the free energy $\Delta^{\prime}$ available in the current profile is negative, i.e., stabilizing, (2) the bootstrap current is helically perturbed reinforcing the perturbation, and (3) the metastable plasma without an island, is sufficiently perturbed above a threshold so that the island grows and saturates [12].

In an AT plasma without sawteeth or fishbones, ELMs could still be present and might cause seed perturbations to excite resonant $q=m / n$ mode such as $m / n=5 / 2$ at $\rho=0.92$ in ARIES-AT [3]. The large bootstrap current can produce a very large island if excited and allowed to grow. However, current profile control with radially localized off-axis co-electron cyclotron current drive (ECCD) or lower hybrid current drive (LHCD) could suppress the island by either replacing the "missing" bootstrap current [13] or by making $\Delta^{\prime}$ more negative [14].

Stability is determined by analyzing the modified Rutherford equation including the effect of replacing the "missing" bootstrap current, $J_{r f} / J_{b s}$ term, and with the "polarization" threshold $\omega_{\text {pol }}$ term [12]:

$$
\frac{\tau_{R}}{r} \frac{d w}{d t}=\Delta^{\prime} r+\sqrt{\epsilon}\left(L_{q} / L_{p}\right) \beta_{\theta}\left[\frac{r}{w}-\frac{r w_{p o l}^{2}}{w^{3}}-\frac{8 q r \delta}{\pi^{2} w^{2}} \frac{\eta J_{r f}}{J_{b s}}\right] .
$$

Here $J_{b s}=\sqrt{\epsilon} p /\left(L_{q} B_{\theta)}, J_{r f}=I_{r f} /(2 \pi r \delta), \delta\right.$ is the full radial width half maximum of the rf current, and $\eta=\eta_{0} /\left(1+2 \delta^{2} / w^{2}\right)$ with perfect positioning on the island assumed. The unstable region is bounded in $\beta_{\theta}-w$ space for $\dot{w} \equiv 0$, as given by

$$
\beta_{\theta}=\frac{-\Delta^{\prime} r}{\sqrt{\epsilon}\left(L_{q} / L_{p}\right)} /\left[\frac{r}{w}-\frac{r w_{p o l}^{2}}{w^{3}}-\frac{8 q r \delta}{\pi^{2} w^{2}} \frac{\eta J_{r f}}{J_{b s}}\right]
$$

Evaluation of the unstable region for the ARIES-AT equilibrium is shown in Figure 8. For $m / n=5 / 2, \Delta^{\prime} r=-10$ is very stable [15]. However, without rf, an unacceptably large island, $w / r \sim 0.5$ at $\beta_{\theta}=2.3$, could be excited.

The feasibility of using radially localized ECCD to replace the missing bootstrap current, at fixed $\Delta^{\prime}$, is also shown in Figure 8. This is seen to be ineffective even for $I_{r f} / I_{p}=0.1$ (assuming $\delta=\sqrt{3} w_{\text {pol }} \approx 6 \mathrm{~cm}<w$ for good efficiency of $\eta=0.5$ ) as $J_{r f} / J_{b s}=0.3$ and about $J_{r f} / J_{b s} \approx 1 \sim 2$ is needed, this corresponds to a large ECCD requirement. $\left(I_{r f} / I_{p} \approx 0.3 \sim 1.0\right)$.

Allowing the equilibrium current density to be modified by the radially localized ECCD or LHCD so as to make $\Delta^{\prime}$ more negative, improves the situation greatly. 
As shown in Figure 8, it requires $\Delta^{\prime} r<-50$ to limit the mode at $\beta_{\theta}=2.3$. The feasibility of this is currently being investigated.

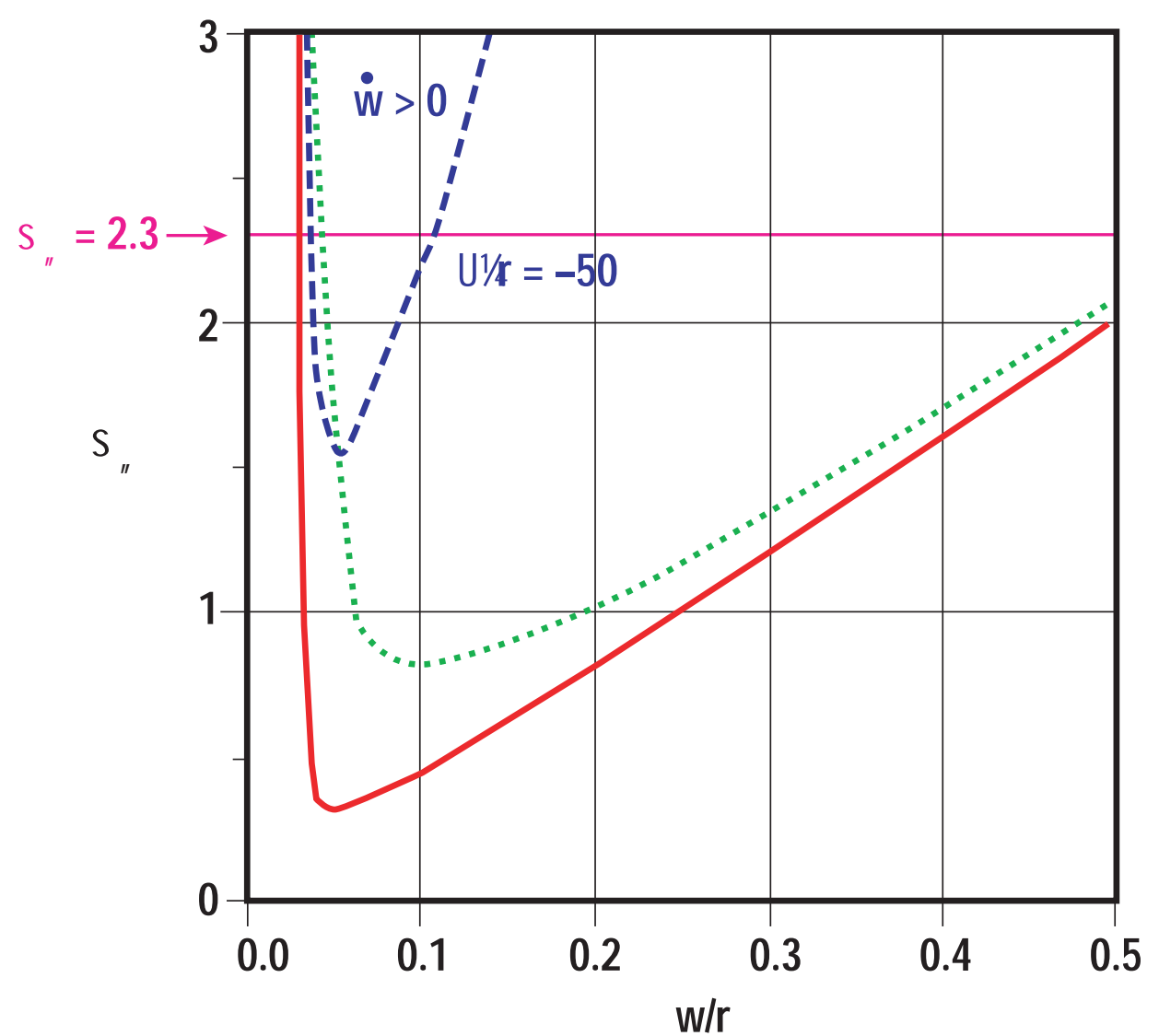

Figure 8: Unstable region $\dot{w}>0$, shrinks slightly at $\Delta^{\prime} r=-10$ with a very large rf current applied $\left(10 \%\right.$ of $\left.I_{P}\right)$ or more effectively if $\Delta^{\prime} r$ is made more negative.

It seems clear that a combination of current profile modification and a replacement of the missing bootstrap current is most effective. Future work should include: (1) equilibrium constructions with local current density bumps such as in [14] for evaluation of $\Delta^{\prime}$ with "TEAR" or PEST-III, (2) analysis of the validity of Eq. (2) under the ARIES-AT conditions, (3) evaluation of rf power and launcher requirements for a multi-MA off-axis ECCD system, and (4) analysis of the feasibility of producing the required localized current profile modifications with a LHCD system. Another part of the strategy is to avoid large-ELMs and other ideal MHD modes, and thus avoid triggering of the NTM. The above discussed LHCD and ECCD can then be used as a backup for the control of occasional triggered islands from unusual events. 


\subsection{Edge Stability and Edge Localized Modes (ELMs)}

In addition to low toroidal mode number (low- $n$ ) global kink modes and high- $n$ ballooning instabilities, high performance AT plasmas can also be subject to edge localized MHD instabilities in the intermediate range of toroidal mode numbers $(3<n<30)$. These modes have been proposed as a model for ELMs and pedestal constraints in the "peeling-ballooning" model (see for example Refs. $[16,17]$ ). These instabilities are driven by a combination of the sharp pressure gradients and the resulting high bootstrap current densities in H-mode type edge profiles. The calculated characteristic mode structure shows a combination of ballooning, peeling, and kink-like features. Depending on radial mode width and other characteristics, these intermediate- $n$ edge instabilities can be associated with small benign ELMs, large ELMs which pose potential heat load issues for the inner wall and divertor, and broader edge instabilities which can inhibit core performance.

An analysis of intermediate- $n$ edge instabilities has been carried out using the ELITE MHD stability code [18]. ELITE solves the edge ballooning equations, which incorporate the effects of edge current and a proper treatment of the plasma-vacuum boundary. The standard L-mode ARIES-AT equilibrium has been analyzed with ELITE and found to be stable to edge modes over a wide range of intermediate mode numbers $(10<n<25)$. This result is consistent with expectations, because the equilibrium has low current density and pressure gradients in the edge region.

The H-mode ARIES-AT equilibrium described in Section 2.2.1 is much closer to edge instability boundaries. Though the equilibrium is stable to pure ballooning modes, the additional "peeling" drive provided by the finite edge current drives this equilibrium close to the marginal point for intermediate $n$ stability. ELITE finds that modes with a radial structure similar to that shown in Figure 9 are marginal at certain intermediate values of $n \sim 15$. These localized instabilities may lead to ELMs as the edge gradients are driven up by transport from the core.

While much recent progress has been made in understanding ELMs, determination of the precise ELM structures and dynamics to be expected in ARIES-AT will require further advances in physics understanding.

\section{Current Drive}

\subsection{Overview}

One of the major physics considerations when designing ARIES-AT is how to maintain the high-performance target equilibrium with a minimal amount of external power, which enhances the attractiveness of the power plant. At the present level of physics understanding, the key lies in our ability to access that class of equilibria where a large portion $(\sim 90 \%)$ of the plasma current is self-driven due to the neoclassical bootstrap effect. Target equilibrium with high values of $\left(\beta_{N}\right)$ and with

high bootstrap fraction have been identified in Section 2. These equilibrium require 


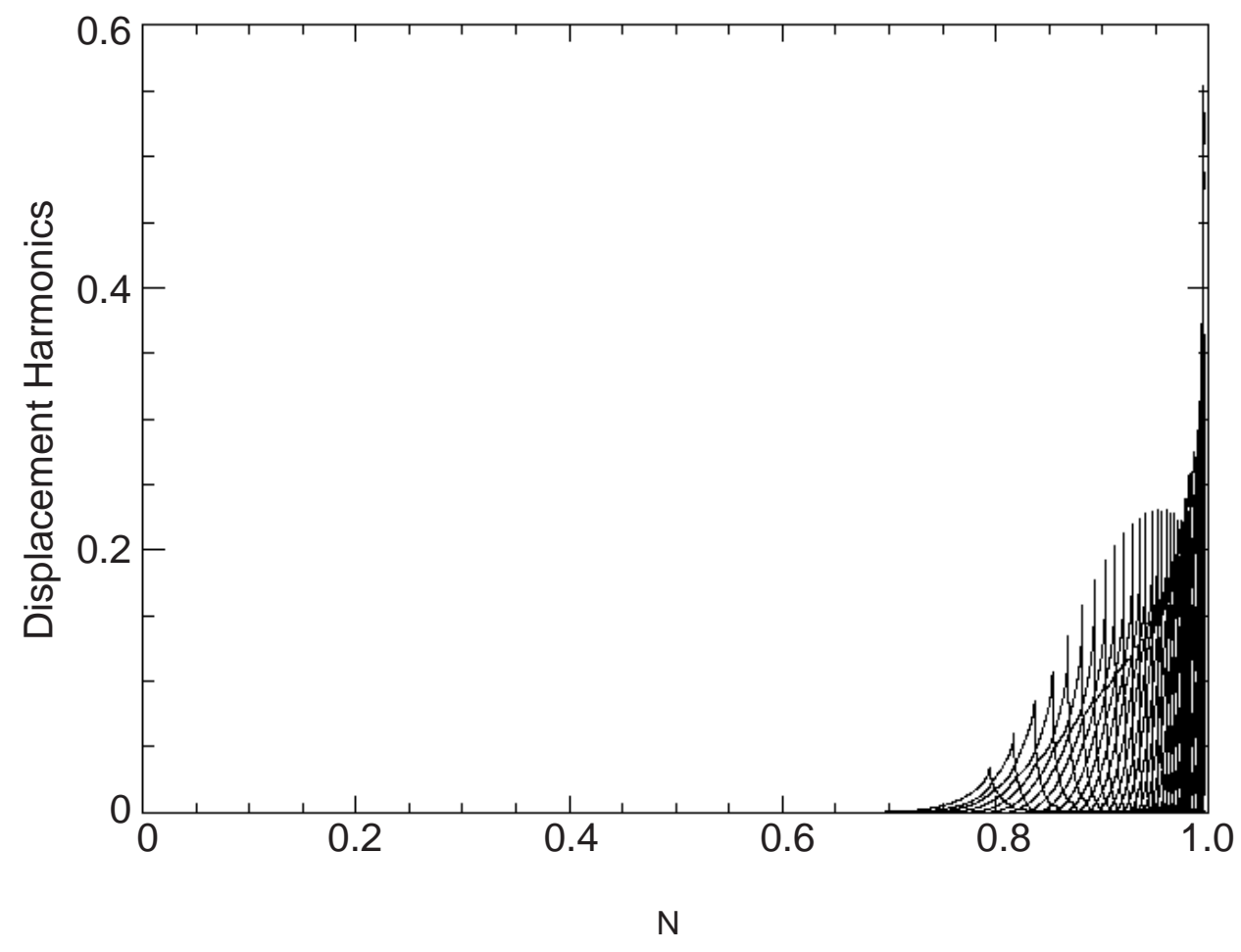

Figure 9: The radial eigenmode structure of a marginally stable $n=16$ mode is shown as function of normalized poloidal flux. The mode structure is calculated by the ELITE ideal MHD stability code using the reference H-mode ARIES-AT equilibrium. (Plotted are the amplitudes of harmonics $42<m<89$.) 
external current drive to maintain the required current profile.

We have used the reference ARIES-AT equilibrium listed in Table 1 to determine the current drive requirements for a number of operating points. In Fig. 10, the current drive contributions are shown for a design point with the plasma parameters: $n_{e o}=2.93 \times 10^{20} \mathrm{~m}^{-3}, n_{o} /<n>=1.36, T_{e o}=26.3 \mathrm{keV},<T_{e}>_{n}=18.25 \mathrm{keV}$ (density weighted volume average electron temperature), and $Z_{\text {eff }}=1.9$ using Neon as the impurity species. Two radio frequency (RF) systems are used to drive the seed currents in order to maintain the target equilibrium current profile. Plotted are the intrinsic and externally driven components of the plasma current in the toroidal direction. About $91 \%$ of the plasma current is self-driven, comprising the bootstrap current $(87 \%)$ and the diamagnetic current (4\%). The bulk (1.1 MA) of the seed current is driven in the outer part of the plasma $(\rho>0.8)$ by waves in the lower hybrid (LH) frequency range, while a small on-axis component $(0.15 \mathrm{MA})$ is driven by fast magnetosonic waves in the ion cyclotron range of frequencies (ICRF). As indicated in Fig. 10 the target equilibrium current profile is well reproduced by the sum of the intrinsic and RF-driven currents. In this optimized scenario, the total RF power requirement is determined to be $41.6 \mathrm{MW}$, with $36.9 \mathrm{MW}$ in the LH system and 4.7 MW delivered by the ICRF system.

In the remainder of this section, we discuss the rationale for selecting the two RF current drive schemes for ARIES-AT, and how we determine the power requirement in each system. During the early stages of this design study, we have also studied alternate ways to drive the seed currents, i.e. the non-bootstrap part of the total current required for equilibrium. These are high-harmonic fast waves (HHFW), neutral beam injection (NBI) and electron cyclotron (EC) waves. The power requirements for these techniques will be compared to the reference systems in the context of possible backup application to ARIES-AT.

\subsection{ICRF Fast Wave System for On-Axis CD}

The requirement for on-axis seed current drive in an ARIES-AT equilibrium is quite modest, with about $0.15 \mathrm{MA}$ ( or $1.1 \%$ of $I_{p}$ ) to be driven within the region $\rho<$ 0.6 , where $\rho$ is the normalized toroidal flux. Because the required power will be modest, the current drive efficiency is not a crucial consideration. However, a broad deposition profile peaked on axis will be required, and the usual criteria of high source efficiency, adequate experimental data base and auxiliary heating role during startup have been taken into consideration.

The two most viable wave candidates for this purpose would be ICRF fast waves and EC waves. On-axis current drive has been demonstrated on a number of tokamaks for both ICRF [19] and EC [20] techniques. Fast wave has a natural tendency to focus towards the density peak on axis where the wave energy is absorbed and a current is driven. It uses conventional technologies in the form of tetrodes and coaxial transmission lines, with a projected power delivery efficiency of $75 \%$. On the other hand, by a suitable choice of frequency (e.g., $f=2 f_{c e}$ on axis), EC wave 


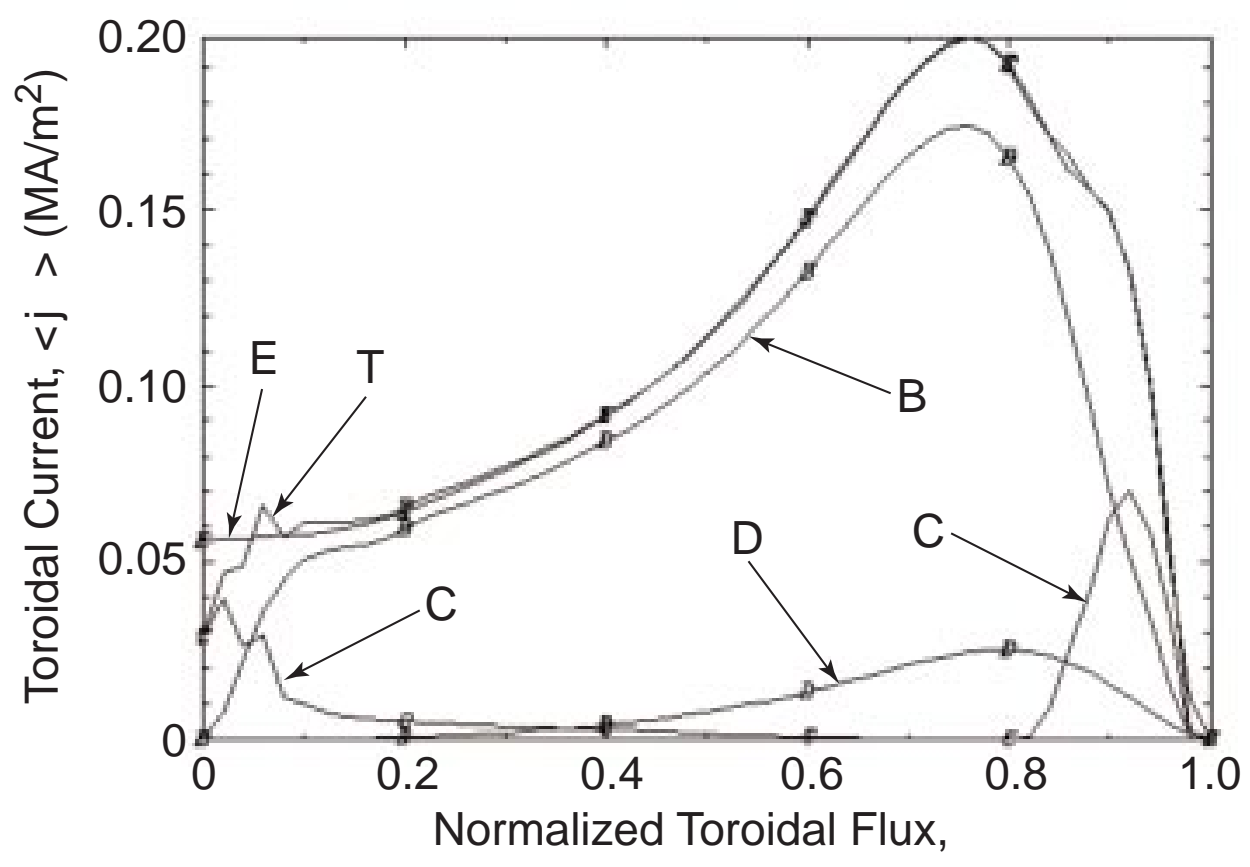

Figure 10: Toroidal components of the bootstrap (B), diamagnetic (D), RF-driven (C) current densities vs. normalized toroidal flux for ARIES-AT. The equilibrium current profile (E) is well reproduced by the driven current $(\mathrm{T})$. 
can drive current on axis in a localized fashion. However, considerable development in the gyrotron source technology [21] will be needed in order to reach steady-sate power efficiencies close to those found in the ICRF system. For ARIES-AT, we have retained ICRF fast waves as the reference on-axis current driver as in previous design studies of ARIES-I [22] and ARIES-RS [2]. This selection is based primarily on the projected higher power efficiency of the ICRF system. As its system performance continues to improve, the EC current drive technique should deserve serious consideration in the next generation of tokamak power plant studies.

Fast waves drive a current by pushing electrons along the magnetic field line when the Landau resonance condition: $\omega-k_{\|} v_{\|}=0$ is satisfied, where $\omega$ is the wave radian frequency, and $k_{\|}$and $v_{\|}$are the wavenumber and electron velocity, respectively, along the total magnetic field direction. When wave power is launched preferentially in one toroidal direction, electrons are accelerated asymmetrically giving rise to a net driven toroidal current. To optimize the current-drive (CD) efficiency, we need to choose wave parameters that ensure strong electron damping in one radial transit while minimizing or avoiding competing wave absorption by ions.

A prudent choice of the wave frequency is the key to avoiding absorption by thermal fuel ions and by energetic alpha particles. Shown in Fig. 11 are the cyclotron frequencies in the first few harmonics range for deuterium, tritium and helium along the mid-plane major radius. Noting that the single-pass electron absorption will be strong in the ARIES-AT regime, the range of acceptable frequencies is determined by the absence of cyclotron resonance on the out-board side of the magnetic axis while allowing for ion resonances towards the in-board edge of the plasma cross section. As displayed in Fig. 11, four frequency candidates, namely, 22, 68, 96 and $135 \mathrm{MHz}$, have been identified. We further note that our choice of the folded waveguide as the launching structure and the need to minimize the radial thickness of the resonant cavity in order to fit into the blanket region dictate higher frequency (shorter wavelength) operation. These engineering constraints lead us to the choice of $96 \mathrm{MHz}$ as the operating frequency for the fast wave system, which places the fourth tritium harmonic outside the out-board edge and the second deuterium (helium) and third tritium harmonics far beyond the magnetic axis on the in-board side. The $22 \mathrm{MHz}$ option is not selected because the required antenna size will be incompatible with fusion core constraints and the data base is sparse, even though this option totally avoids ion absorption. On the other hand, for the $135 \mathrm{MHz}$ option, it is found that electron damping is so strong due to the relatively high electron $\beta$ that the deposition and driven profile become very broad and even hollow, making it unsuitable for on-axis seed current drive.

The CURRAY ray tracing code [24] is the main analysis tool for RF current drive. This code has been benchmarked with the PICES full wave code of Oak Ridge National Laboratory in the context of advanced tokamak scenario evaluation for FIRE and ITER at the 2002 Snowmass Summer Study, and obtains good agreement with the HPRT ray tracing code of Princeton Plasma Physics Laboratory in the case of dominant electron damping in NSTX. In this code, electron absorption 


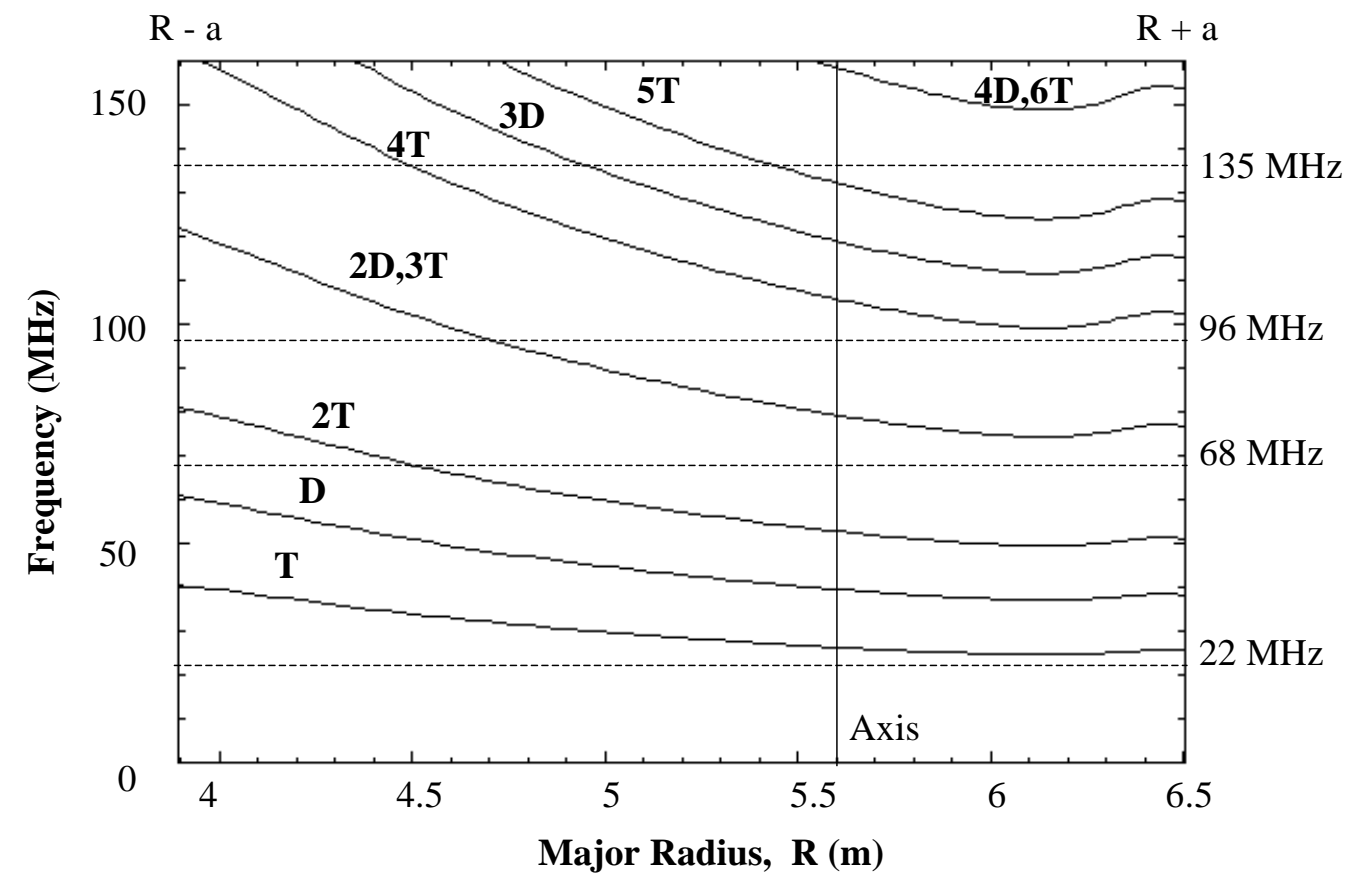

Figure 11: Cyclotron frequencies for D, T and He over the mid-plane major radius for the first few harmonics denoted by number in front of species. Dashed horizontal lines indicate frequency options for fast wave current drive. 
is calculated with the inclusion of electron Landau damping and transit-time magnetic pumping, while fuel ion absorption is computed for the harmonic cyclotron damping processes. For the ARIES-AT parameters, the ray trajectories are calculated using the cold ion approximation, while the ion damping model includes the fully kinetic finite-Larmor-radius effect. Cyclotron damping by the energetic alpha particle damping is calculated using an equivalent Maxwellian temperature for the slowing down distribution function in the absence of RF-induced velocity diffusion. The corresponding density profile is evaluated from the local fusion reaction rate.

The CURRAY code is then used to determine the fast wave launch location on the out-board edge and the launched wave spectrum to obtain the best match of the driven current profile to that of the central seed current. We used fifteen rays to represent the launched wave spectrum with a cosine-squared dependence along the poloidal extent of the waveguide mouth. We found that launching from the out-board mid-plane with a spectrum peaked at $N_{\|}=2$ gives the best matching (used in Fig. 10). Since the wave model used in CURRAY assumes a Maxwellian plasma, by adjusting the incident power level, we determine that $4.7 \mathrm{MW}$ of power is required to drive the $0.15 \mathrm{MA}$ on-axis seed current, thus yielding a CD efficiency of $0.032 \mathrm{~A} / \mathrm{W}$. We have not attempted matching the current profiles exactly because it will result in an unrealistic antenna spectrum. In Fig. 12, we show the wave power propagation pattern, represented by the fifteen rays, projected onto the minor cross section and when viewed from the top of the torus. Strong single-pass absorption is evident from Fig. 12(a) and the wave is confined to the vicinity of its toroidal launch location. Importantly, it is found that $99.4 \%$ of the launched power is absorbed by electrons, with the rest going to alpha particles and tritium. So there is negligible ion absorption of the wave, including impurity ions, in this reference scenario.

The fast wave power is coupled to the plasma via a launcher module similar to the ARIES-RS folded waveguide design [23], to take advantage of its structural robustness and projected high power handling capability. This module is located on the outboard midplane, and consists of four waveguides in a toroidal array, fed with a $90^{\circ}$ phase difference. Each waveguide has ten folds, and with a capacitive diaphragm along each vane, has a radial thickness of $0.92 \mathrm{~m}$. The whole module has a toroidal width of $1.56 \mathrm{~m}$ and a poloidal height of $0.51 \mathrm{~m}$, occupying a first wall area of $0.80 \mathrm{~m}^{2}$. Using a conservative power limit projection of $25 \mathrm{MW} / \mathrm{m}^{2}$, the launcher should be able to deliver $15 \mathrm{MW}$ of power to the plasma.

\subsection{Lower Hybrid System for Off-Axis CD}

For the reference ARIES-AT equilibrium presented in Sec. 3.1, the bulk of the seed current must be driven off axis, $0.8<\rho<1$, with a profile peaked at $\rho \approx 0.85$. We have examined a variety of radio frequency CD options including LH waves, EC waves and high harmonic fast waves (HHFW). Of these, lower hybrid waves have been studied most extensively, with a large experimental data base [25] that includes demonstration of off-axis drive to reach a reverse-shear configuration on 

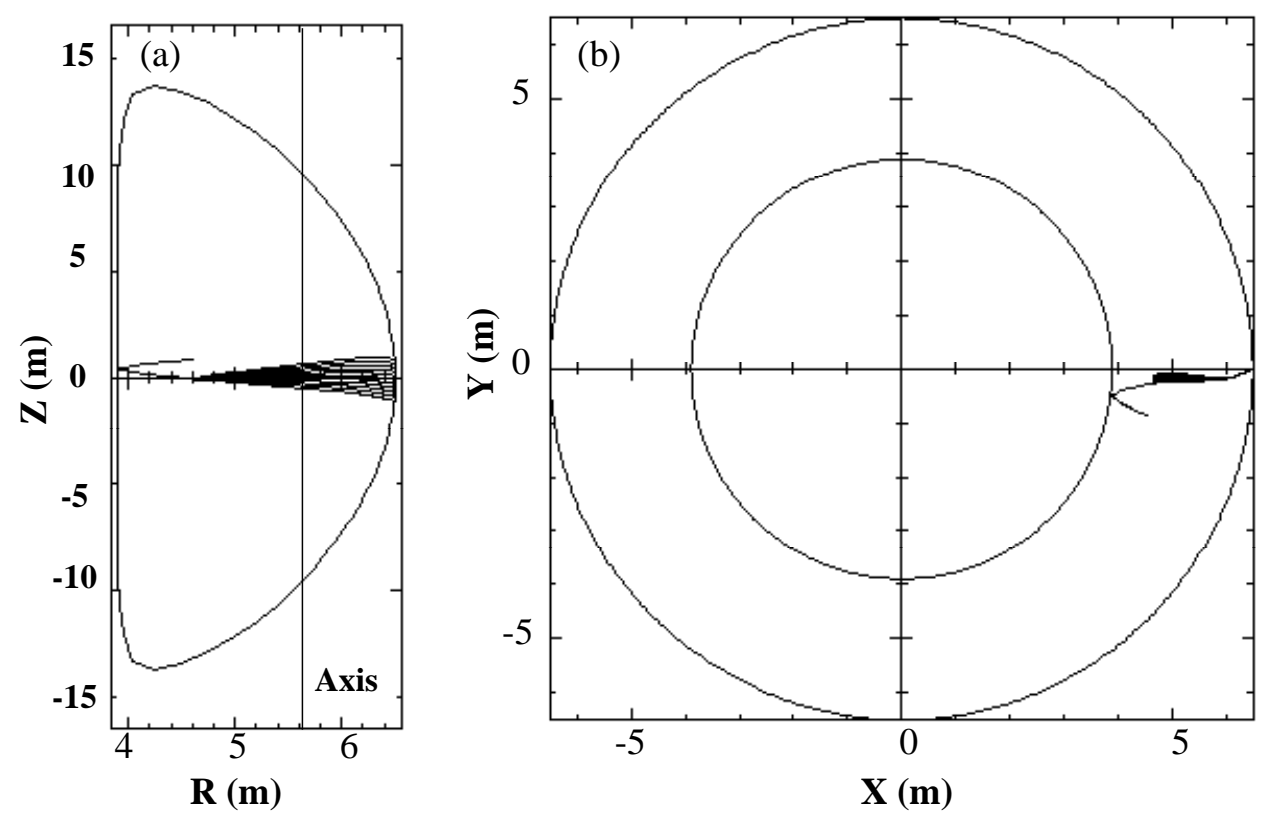

Figure 12: Fast wave ray trajectories projected on (a) minor cross section, and (b) top view of torus. 
Tore Supra and sustainment of hour-long discharges on TRIAM-1M [26]. In reactorgrade plasmas, the wave typically propagates into the plasma and becomes totally damped before reaching the plasma core. Because of the strength of the damping, the deposition profile tends to be highly localized in space, making it quite suitable for profile control. Since the wave is resonantly absorbed by super-thermal electrons $\left(\omega / k_{\|} v_{e} \simeq 3\right)$, its CD efficiency is highest amongst all RF techniques. For off-axis drive, because of the paucity of trapped electrons in the resonant velocity spectrum, degradation in CD efficiency due to neoclassical effects will be minimal.

Fast waves in the high harmonic regime $\left(f / f_{c i} \sim 20\right)$ can also be considered for off-axis drive as suggested in ARIES-RS [2]. The data base for this wave is very sparse, even as recent HHFW heating results on the NSTX spherical tokamak [27] were reported, indicating electron heating in the plasma $\beta$ range of $5-10 \%$. The wave damps on electrons with $\omega / k_{\|} v_{e} \sim 2$, thus allowing deeper penetration into the plasma core than LH waves. However, in the core region, energetic alphas are a source of competing wave damping mechanism which effectively reduces absorption by electrons. Also, there is only modest degradation in CD efficiency due to particle trapping. The deposition profile for a single launched $k_{\|}$wave spectrum will be broad making it difficult for detailed profile control. Our calculations for ARIESAT indicated that, for the highest CD efficiency, the current would be driven in the mid-plasma region, i.e., $\rho<0.8$, which is not where the off-axis seed current is located. This point will be further explored in a following subsection.

We have also investigated the possibility of using electron cyclotron waves to drive off-axis current on ARIES-AT. This has recently been demonstrated on DIIID. [20] As previously mentioned, sources (gyrotrons) in the EC regime are comparatively less efficient than those (klystrons) in the LH and HHFW frequency ranges, which makes the EC wave less attractive, particularly when a substantial amount of current needs to be driven. In addition, for off-axis drive near the outer plasma periphery, where the trapped particle population is high, the EC current drive efficiency will be strongly degraded. Based on this qualitative argument, we have excluded EC waves from consideration for off-axis drive on ARIES-AT.

We determine the LH wave system requirements by running the CURRAY code at the slow branch of the wave dispersion relation. Because the off-axis seed current has a relatively broad profile, we found that it is necessary to launch a number of wave spectra, each centered at a different $N_{\|}$, in order to achieve the desired profile. Usually, the wave frequency is determined by requiring total elimination of energetic alpha particle interaction. This leads to a relatively high frequency and short wavelength, particularly for edge CD with high $N_{\|}$, making cooling of the corresponding waveguide grille launcher extremely difficult. Here we argue that since current is to be driven in the outer part of the plasma, where the energetic alpha population should be low, we may lower the frequency requirement without suffering large wave absorption through the alphas. As a result, we are able to use $3.6 \mathrm{GHz}$ for those lower- $N_{\|}$spectra that penetrate deepest into the plasma and 2.5 $\mathrm{GHz}$ for those that drive currents just inside the separatrix. 
First, we generate a core accessibility diagram in terms of the window of accessible local $N_{\|}$along the midplane major radius, using the prescribed $n_{e}$ and $T_{e}$ profiles for the equilibrium, as shown in Fig. 13. For the LH wave to be accessible to a radial location inside the plasma, the local $N_{\|}$must satisfy the condition: $\left[1+\left(f_{p e} / f_{c e}\right)^{2}\right]^{1 / 2}+f_{p e} / f_{c e}<N_{\|}<7 / T_{e}^{1 / 2}(\mathrm{keV})$. For a typical ARIES-AT equilibrium, we found that the LH wave can penetrate as deep as $\rho \approx 0.8$ as shown in Fig. 13.

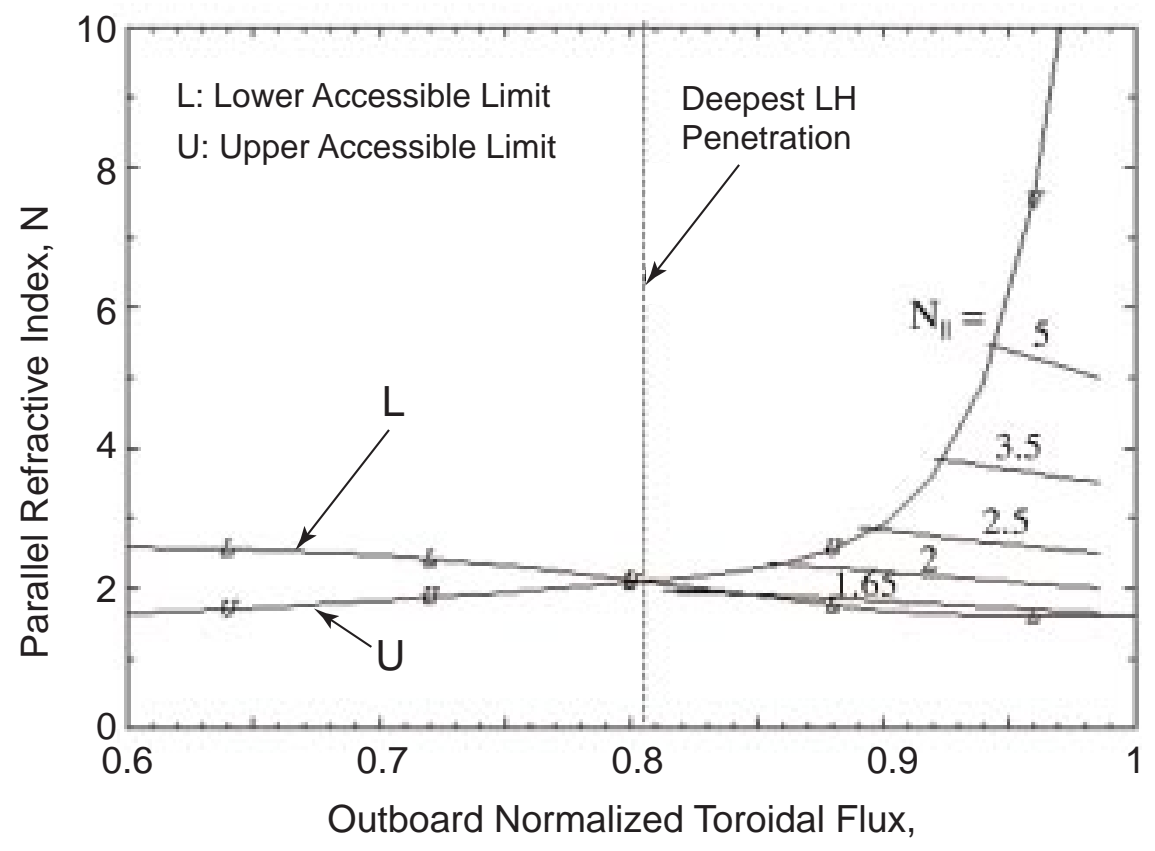

Figure 13: Lower hybrid accessibility $N_{\|}$window for a typical ARIES-AT equilibrium, and evolution of $N_{\|}$along LH ray paths for the launched spectra given in Table 4.

To determine the required launched LH spectrum, we scan a range of launched $N_{\|}$and poloidal locations on the outboard edge in order to maximize the radial penetration and CD efficiency. Since the wavelength is short compared to the energetic alpha gyro-radius and the resonance surfaces are closely packed, we can model wave absorption by energetic alphas using the unmagnetized approach, where the resonance condition is given by $v_{\alpha}=\omega / k$, and an alpha slowing down distribution is assumed. Launching lower $N_{\|}$results in power deposition and current drive in deeper radial locations, and vice versa. However, at the deepest deposition loca- 
tion, we found a small amount ( $1 \%$ ) of alpha particle wave absorption, and none as the deposition moves radially outward as the launched $N_{\|}$is increased. For the reference scenario, we found that it is necessary to launch a broad $N_{\|}$spectrum in order to drive the off-axis seed current profile as indicated in Fig. 10. Details of the launched spectra are given in Table 4, with the driven current profiles for the five $N_{\|}$components and their sum displayed in Fig. 14. It is clear from this that the $N_{\|}$spectrum could be modified slightly to increase or reduce the current drive for $\rho>0.95$ if it was found that this was required for ELM stability.

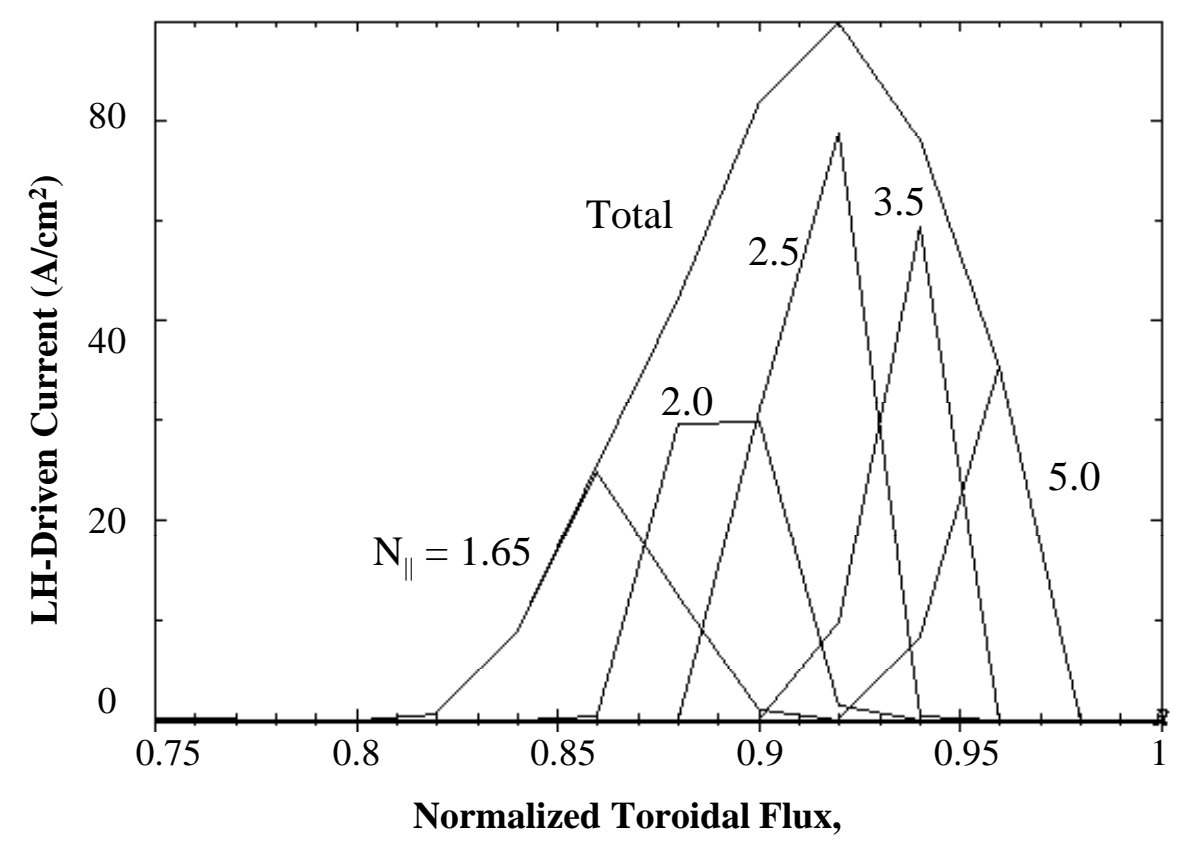

Figure 14: Current profiles driven by various $N_{\|}$components of the launched spectrum.

The evolution of $N_{\|}$along the ray trajectories are shown in Fig. 13. It is clear that radial accessibility of the five launched spectra agrees well with the prediction from the 1-D slab model. In particular, the ray started with the lowest $N_{\|}(=1.65)$ shows relatively little upshift in magnitude as it propagates inward until it encounters a mode conversion point at the lower accessibility limit, from where it turns back before total absorption by electrons. For higher launched $N_{\|}$, the wave energy propagates inward and gets absorbed well before it reaches mode conversion zone.

The electron absorption is calculated assuming weak RF-induced diffusion in 
Table 4:

\section{Lower hybrid current-drive system parameters}

\begin{tabular}{cccccc}
\hline Freq. $(\mathrm{GHz})$ & $N_{\|}$ & $\theta^{\circ}$ & $\mathrm{I} / \mathrm{P}(\mathrm{A} / \mathrm{W})$ & Power $(\mathrm{MW})$ & $I_{c d} / I_{\text {seed }}$ \\
\hline 3.6 & 1.65 & -90 & 0.053 & 3.06 & 0.15 \\
3.6 & 2.0 & -90 & 0.049 & 4.40 & 0.2 \\
3.6 & 2.5 & -90 & 0.039 & 8.22 & 0.3 \\
3.6 & 3.5 & -90 & 0.024 & 8.87 & 0.2 \\
2.5 & 5.0 & -90 & 0.013 & 12.39 & 0.15 \\
\hline
\end{tabular}

velocity space, i.e., the velocity distribution is taken to be Maxwellian near the resonant region, $v_{\|} / v_{e} \approx 3$. This assumption is justified by noting that the local density is very high $\left(n_{e} \sim 0.6 \times 10^{20} \mathrm{~m}^{-3}\right)$, and the temperature is relatively low $\left(T_{e} \sim 5 \mathrm{keV}\right)$, so that thermalization via collisions can be strong. (As a check, we calculated the electron damping strength assuming strong RF-induced diffusion by setting the normalized diffusion coefficient, $D_{r f} / D_{\text {coll }} \approx 0.5$, for which we can approximate the local damping decrement by $\gamma_{e} \simeq \gamma_{e o} /\left(1+\sqrt{2} x_{e}^{3}\right)$, [28] where $\gamma_{e o}$ is the Maxwellian damping decrement and $x_{e}=\omega / k_{\|} v_{e}$. We found that, for the case of launched $N_{\|}=1.65$ in Fig. 13, the penetration depth remains the same, and the deposition profile is peaked slightly inward.)

The LH wave launcher system is composed of five separate modules which are designed to radiate different wave spectra. Each module consists of a toroidal array of alternate passive and active $\mathrm{TE}_{50}$ rectangular waveguides with the short (long) dimension in the toroidal (poloidal) direction. The basic element of the launcher unit is the passive-active multijunction (PAM) grille modeled after the ITER-EDA design [29]. Each PAM grille is driven by a number of $\mathrm{TE}_{10}-\mathrm{TE}_{50}$ mode converters with a projected conversion efficiency of $98 \%$. The modules are located approximately $1 \mathrm{~m}$ below the outboard midplane, and occupy a first wall area of $1.26 \mathrm{~m}^{2}$.

To conclude this section, we point out that off-axis LH current drive offers the prospect of stabilizing neo-classical tearing modes (NTM) which can degrade the achievable $\beta$ limit. The growth of the island width associated with these modes can be suppressed by localized current drive in the vicinity of a rational-q surface. This process has been demonstrated on COMPASS-D [30], where LH current drive at modest levels of power $(60-70 \mathrm{~kW})$ was shown to have a strong stabilizing effect on the $\mathrm{m}=2, \mathrm{n}=1$ neo-classical tearing mode. Reduction of the $(2,1)$ mode amplitude was clearly observed during the LH pulse accompanied by a rise in the plasma $\beta_{p}$, and the mode grew back to its original amplitude after the LH pulse. The neoclassical nature of the mode was inferred from reasonable agreement of the measured island width with that from an acceptable analytical fit. A detailed analysis of NTM 
stabilization by LH waves for ARIES-AT is beyond of the scope of the present work, and it should be a subject of future research.

\subsection{Backup Current Drive Scenarios}

In the previous two sections, we have examined the ARIES-AT baseline reverse shear equilibria where specific plasma profiles have been prescribed to ensure that no penetration of the LH waves inside $\rho \approx 0.8$ is required. Under these conditions, only two RF current-drive systems are required, as contrasted with three systems required on ARIES-RS. As we deviate from these profiles, we find that seed current needs to be driven in the mid-radius region, i.e., $\rho<0.8$, where the LH wave is inaccessible. In this situation, either a third RF system in the high-harmonic frequency regime is required, or a neutral beam injection system can be used, in place of LH waves, to drive the entire off-axis seed current.

\subsubsection{Three RF Systems}

The bootstrap current alignment in an equilibrium is sensitive to the pressure, density and temperature profiles, and to the effective charge of the plasma, $Z_{E F F}$. Current mis-alignment can be caused by mild variations of the profiles and $Z_{E F F}$ from the target values which, when un-checked, will result in the equilibrium evolving towards a less desirable state. Therefore, backup systems may be necessary to provide current drive in a broader region than is possible with the baseline CD systems, in order to continue maintaining the equilibrium. We will illustrate this concept by an example.

Shown in Fig. 15 is a plot of the seed current radial profile for the same equilibrium as in Sec. 3.1, but with $Z_{\text {eff }}=2.0, n_{e o}=2.74 \times 10^{20} \mathrm{~m}^{-3}, T_{e o}=28.2 \mathrm{keV}$, $<T_{e}>_{n}=19.3 \mathrm{keV}$, and $n_{e}, T_{e}$ profiles adjusted to eliminate bootstrap overdrive. We note that the off-axis seed current is now extended to $\rho=0.75$, and a third $\mathrm{CD}$ system is required to drive the seed current in the region $0.75<\rho<0.8$. We propose to use the fast wave in the high-harmonic frequency range $\left(f / f_{c i} \sim 20\right)$ for this purpose, since it penetrates deeper than LH waves because of its relatively weaker electron damping. However, the high-harmonic fast wave is susceptible to strong energetic alpha damping in this mid-radius plasma region as we will show.

The current drive scenario for this case, therefore, consists of three RF systems: (1) ICRF fast wave for central drive, (2) HHFW for mid-radius drive and (3) LH waves for off-axis drive. The required RF system parameters are given in Table 5, and the driven currents are displayed also in Fig. 15, reproducing reasonably well the target seed current profile. For the launched HHFW power of $20.1 \mathrm{MW}$, about $34 \%$ is absorbed by energetic alpha particles, which are modeled as an equivalent thermal species in the CURRAY code. Lowering $N_{\|}$tends to increase the alpha absorption fraction, and vice versa, with moderate shifts in the driven current peak location. The chosen value of $N_{\|}(=2.5)$ corresponds to the highest CD efficiency 
while driving currents in the required location.

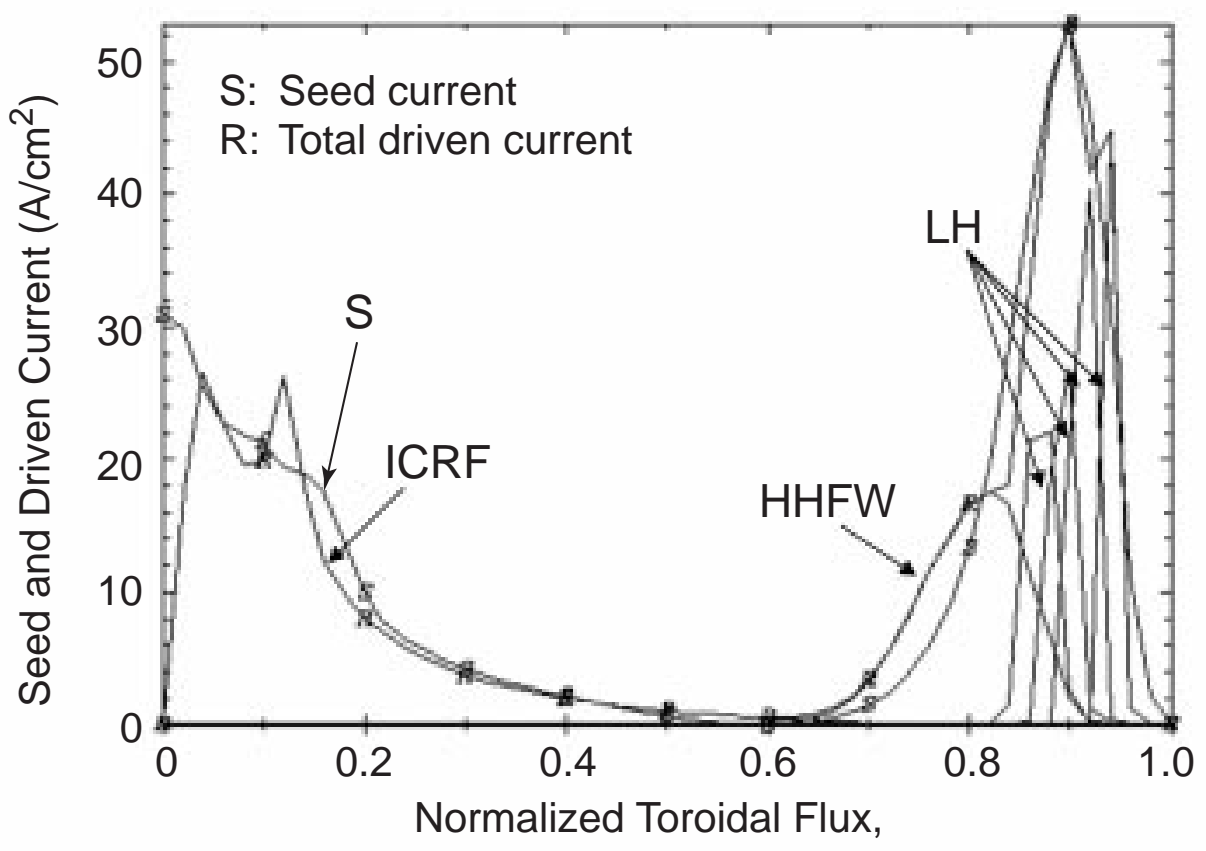

Figure 15: Alignment of RF driven current profile with that of the seed current. Current profiles driven by the three RF systems are also shown.

\subsubsection{Neutral Beam System}

For the case shown in the previous subsection, we may also consider replacing HHFW and LH waves with a single tangential neutral beam injection (NBI) system to drive the off-axis seed current. The primary motivation for investigating NBI current drive is that the beam-imparted toroidal momentum also drives a significant amount of rotation that may contribute to stabilizing the external kink mode. As shown in Fig. 16, the deepest penetration required for off-axis drive is $\psi=0.35$ where $\psi$ is the normalized poloidal flux, which translates to $\rho=0.75$ as in Fig. 15. Using the NFREYA neutral beam deposition code [31], we found that a beam energy of $120 \mathrm{keV}$ at a pivot angle of $70^{\circ}$ is sufficient for the penetration. With an injected power of $44 \mathrm{MW}$, a driven current profile that matches the off-axis seed is obtained, at a CD efficiency of $0.026 \mathrm{~A} / \mathrm{W}$. Together with the on-axis fast wave CD power of $4 \mathrm{MW}$, the total current-drive power is $48 \mathrm{MW}$. In principle, the required NBI 
Table 5:

Parameters for three CD system scenario

\begin{tabular}{lccccc}
\hline System & Freq. (GHz) & $N_{\|}$ & $\theta^{o}$ & $\mathrm{I} / \mathrm{P}(\mathrm{A} / \mathrm{W})$ & Power $(\mathrm{MW})$ \\
\hline ICRF/FW & 0.07 & 2.0 & -15 & 0.034 & 4.0 \\
HHFW & 2.5 & 0 & 0.019 & 20.1 & \\
LH & 4 & 1.7 & -90 & 0.057 & 2.94 \\
& 4 & 2.0 & -90 & 0.052 & 3.24 \\
& 4 & 2.5 & -90 & 0.041 & 5.43 \\
& 4 & 4.0 & -90 & 0.021 & 8.17 \\
\hline
\end{tabular}

power should be even higher because the NFREYA analysis here has not taken the trapped particle effect into account in calculating the fast ion current. Nevertheless this investigation indicates that if NBI were used, the CD power would be higher than using three RF systems. For these reasons, we do not consider it further.

\subsection{Current Drive Efficiency Scalings}

While the MHD equilibrium and stability studies in Subsections 2 and 3 provide the optimum range of plasma performance for ARIES-AT, current drive analysis discussed in this section is aimed at providing the range of CD power required to sustain the equilibrium at various operating points, from which an optimum design point can be deduced. The key parameter, or figure of merit, for consideration is the normalized current-drive efficiency, defined as $\gamma_{B}=\bar{n}_{e} I_{p} R / P_{C D}$, where $\bar{n}_{e}, I_{p}$, $R$ and $P_{C D}$ have units of $10^{20} \mathrm{~m}^{-3}, \mathrm{MA}, \mathrm{m}$, and $\mathrm{MW}$, respectively. Note that in this definition, the contribution from the bootstrap current is taken into account, even though there is no CD power associated with it. The usual definition of CD efficiency in the literature is recovered by $\gamma_{C D}=\left(1-f_{B S}\right) \gamma_{B}$.

We note that $\gamma_{B}$ is essentially a function of temperature $T_{e}$ and plasma $Z_{e f f}$, after the density and major radius are factored out. The efficiency of the current drive is dependent on $T_{e}$ and $Z_{e f f}$, while the bootstrap fraction is governed by the aspect ratio, $\beta_{p}, Z_{\text {eff }}$ and details of the plasma profiles. For a fixed equilibrium, selfconsistent variation of the $(n, T)$ profiles and $Z_{\text {eff }}$ results in varying $f_{B S}$ and current drive power requirements. This gives rise to a scaling of $\gamma_{B}$ with respect to $T_{e}$ and $Z_{\text {eff }}$ for the same equilibrium. The $Z_{\text {eff }}$ dependence is important in determining the optimum core radiation power fraction required to lower the divertor surface heat flux without compromising the overall power plant performance.

We have investigated two approaches to obtaining a CD efficiency scaling for use in the ARIES-AT systems analysis in order to determine an optimum operat- 


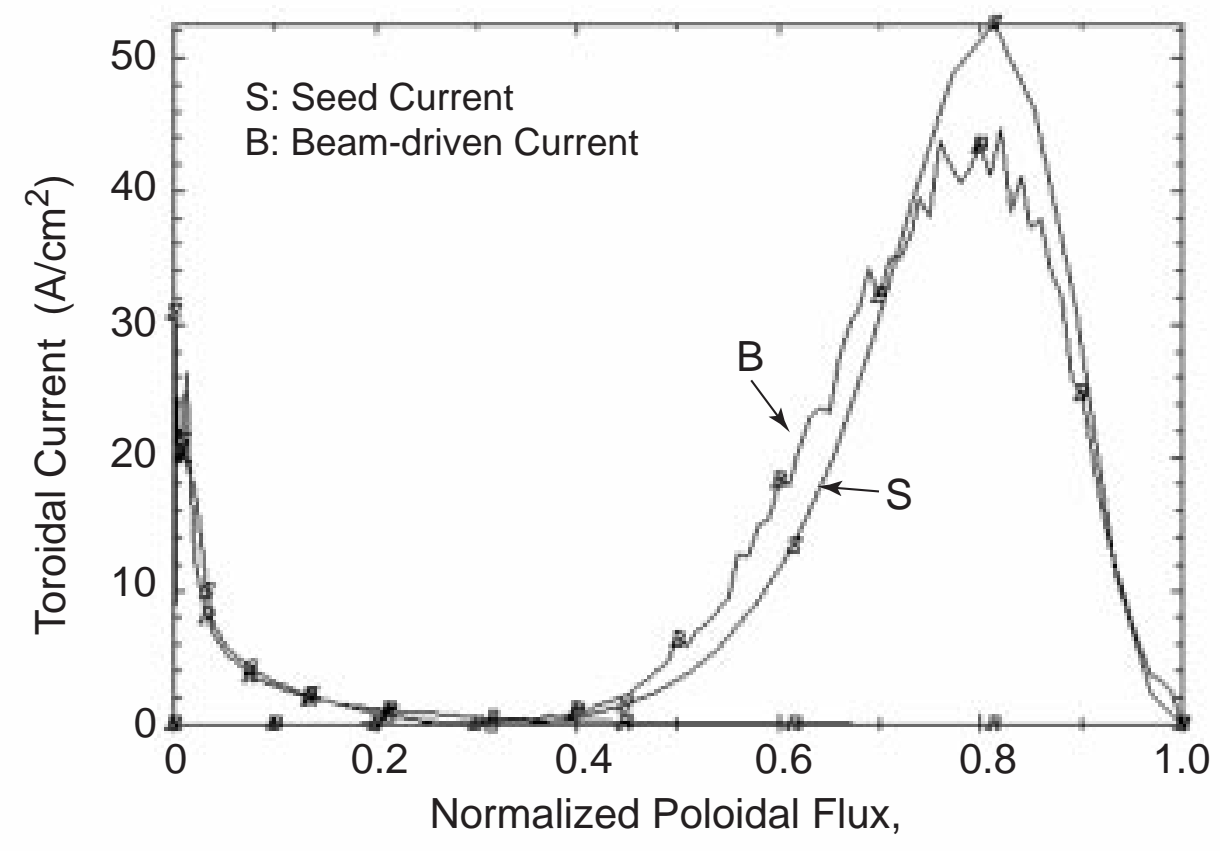

Figure 16: Alignment of beam-driven current profile with that of the seed current. Equilibrium and plasma conditions are the same as in Fig. 15. 
ing point. In the first approach, we simply vary the $(n, T)$ profiles and $Z_{\text {eff }}$ selfconsistently from a reference plasma parameter set for a fixed equilibrium, while avoiding localized bootstrap current overdrive. LH and ICRF power requirements are then determined for a range of relevant $T_{e}$ and $Z_{\text {eff }}$ values. As such, the bootstrap fraction varies for each of these points and may not be optimum. In Fig. 17, we show the resultant normalized CD scalings versus $T_{e}$ for $Z_{e f f}=1.6,1.7$ and 1.8 . As can be seen, the CD efficiency is peaked at $Z_{\text {eff }}=1.7$, drops slightly at $Z_{\text {eff }}=1.6$, but is drastically reduced at $Z_{e f f}=1.8$. It is not surprising that $\gamma_{B}$ optimizes at $Z_{\text {eff }}=1.7$ since the bootstrap alignment is optimized at this value of the effective charge. It is further noted that for $Z_{\text {eff }}=1.8$, three RF systems (ICRF $+\mathrm{HHFW}+\mathrm{LH}$ ) are actually required to drive the seed currents, leading to a substantially lower $\gamma_{B}$ value.

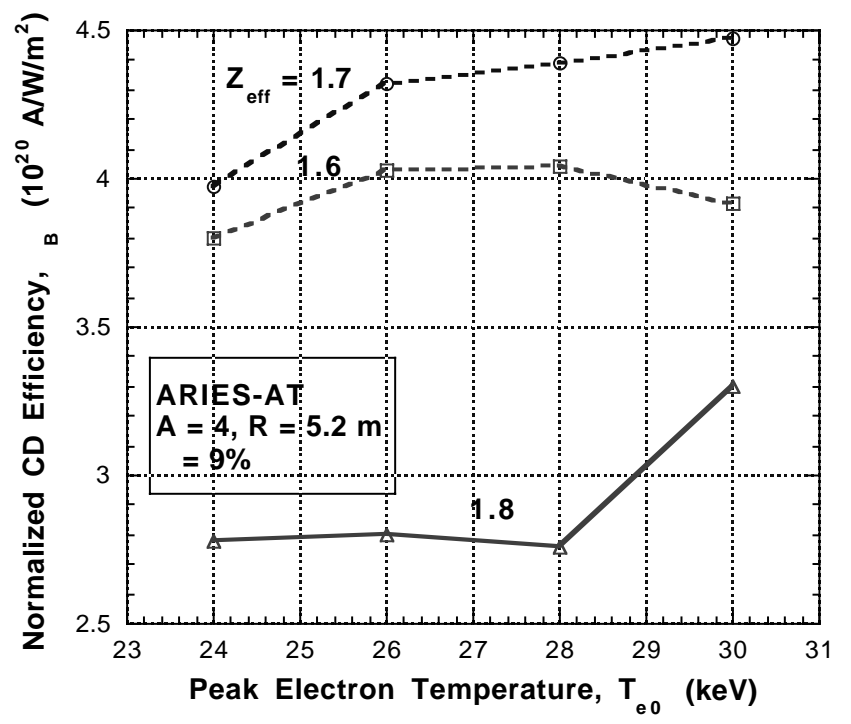

Figure 17: Normalized current-drive scalings with plasma temperature and effective charge for a typical ARIES-AT equilibrium.

Reduction of the divertor heat load often dictates that the plasma operates at $Z_{\text {eff }} \sim 1.8-2.0$. Therefore, it is crucial that at each operating point considered the bootstrap alignment is optimized to minimize the CD power required. Thus, a series of test equilibria have been obtained which maintain the same global plasma 
parameters such as $\beta$, size and shape, but which have optimum bootstrap current alignment requiring only two $\mathrm{RF}$ current-drive systems (ICRF $+\mathrm{LH})$. The resulting CD scalings with $T_{e}$ for three values of $Z_{\text {eff }}$ are shown in Fig. 18. It is clear from the figure that $\gamma_{B}$ is higher than that shown in Fig. 17 because of the optimized bootstrap alignment, and only two RF systems are used. Again the CD efficiency is peaked at $Z_{\text {eff }}$ and drops off from there. This set of CD efficiency scalings has been incorporated into the systems analysis to generate the reference design point for ARIES-AT.

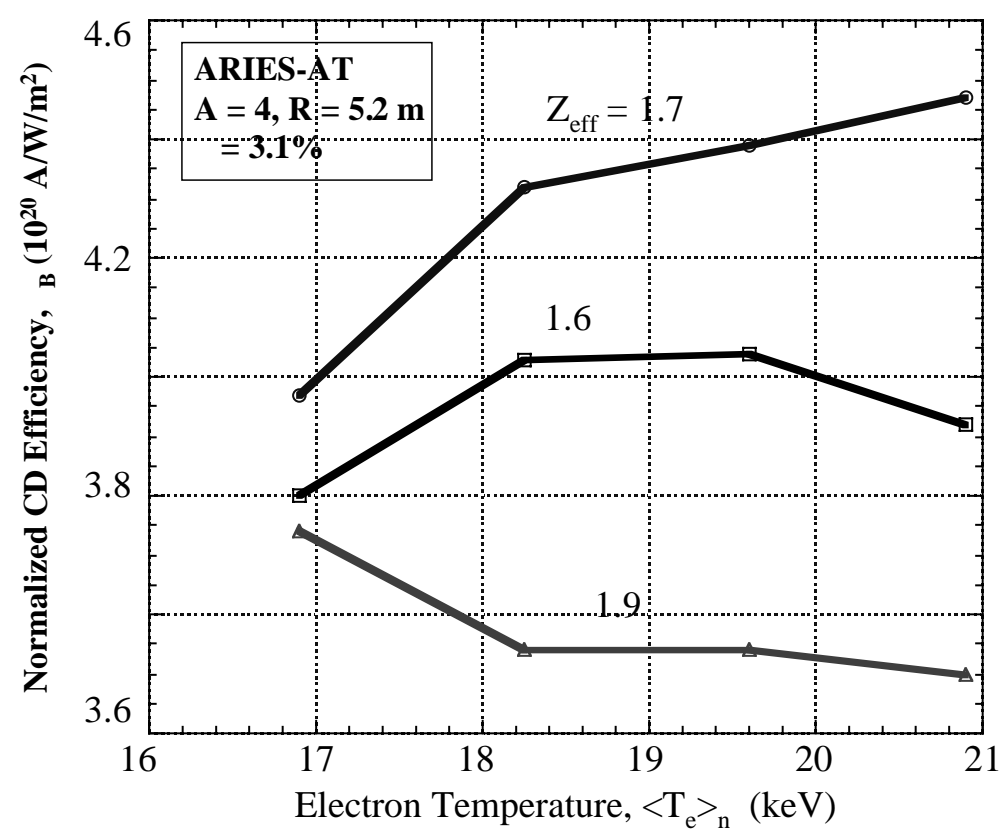

Figure 18: Normalized current-drive scalings with plasma temperature and effective charge for similar equilibria with optimized bootstrap alignment. 


\section{Transport Analysis and Energy Confinement}

\subsection{Transport Studies Using GLF23}

\subsubsection{Introduction}

Previous reactor design studies have predicted the transport for the device by doing a "0D" fit to a database of existing tokamaks. However, in recent years an alternative method has become available with the advent of drift-wave based transport models. For the ARIES-AT design the GLF23 drift-wave based model [33] has been used to project the transport. This model has been shown to be about as accurate as the empirical scaling in predicting the global energy confinement time for a database of tokamak discharges [34] which included both L-mode and H-mode discharges. The GLF23 transport model takes no fitting parameters from experiment. It has been constructed to fit to a set of theoretical calculations. The model computes the linear growth rates for drift-wave instabilities (ion temperature gradient modes ITG, trapped electron modes TEM, and electron temperature gradient modes ETG). The growth rates are found using trial wave-functions and the approximate gyro-Landau fluid (GLF) equations [35]. The trial wave-functions were chosen to give a good fit to the exact gyro- kinetic linear growth rates [36] over a range of local plasma parameters. The linear eigenmodes are then used in a quasilinear calculation of the fluxes (energy, particle, viscous stress) due to turbulence. A model for the saturated turbulence fluctuation level is used to give a fit to a limited set of nonlinear GLF turbulence simulations [37]. For this study the edge values are at the separatrix and do not influence the results much. The prediction of the density profile with the GLF23 model has not been compared with experiment as extensively as the predictions of the temperature profiles, and so in the following sutdies we fix the density profile and evolve only the temperatures to find parametric dependences.

Thus, the model does not use any fitting parameter to experiment but is only fit to more exact theoretical calculations. In principle the GLF23 model has greater predictive power when extrapolating to new tokamaks than empirical scaling laws. However, the limited range of parameters over which the model was fit to theory limits the range of extrapolation which is possible. These limitations will be brought up as required in discussing the GLF23 modeling results for ARIES-AT.

\subsubsection{Analysis}

Three cases will be discussed. The ion and electron temperatures and the toroidal and $E \times B$ velocities are evolved but the density profile is prescribed. Thus, the three cases involve variations of the density profile, but keeping the pressure profile fixed as given in Eq. 1 (with modifications near the boundary as described in Sec. 2.2.1. The first has a peaked density profile close to that used for the bootstrap current calculation in the MHD equilibrium. The surprising thing about this case is that the energy confinement is better than the empirical H-mode scaling but there is no 
edge (or core) transport barrier due to $E \times B$ velocity shear. The reduced transport is predicted to be due to the large Shafranov shift and the negative magnetic shear. This case results in a very good agreement between the MHD stability optimized pressure profile and the pressure profile computed from GLF23.

The second and third cases have flatter density profiles. The main difference between them is the boundary condition used for the $E \times B$ velocity at the separatrix. For all three cases the fusion power and neutral beam deposition was computed using the ONETWO [38] transport code. The steady state temperature profiles were then computed using the XPTOR [39] code, which has special numerical methods designed for the GLF23 model. These temperature profiles were then used to recompute the sources in ONETWO. This iteration procedure converges quickly. The fusion power was reduced if necessary by changing the ion isotope mixture in order to keep the total plasma stored energy below the design target. The toroidal rotation profile was also computed assuming $50 \mathrm{MW}$ of $120 \mathrm{Kev}$ co-injected neutral beams with the torque density computed in ONETWO.

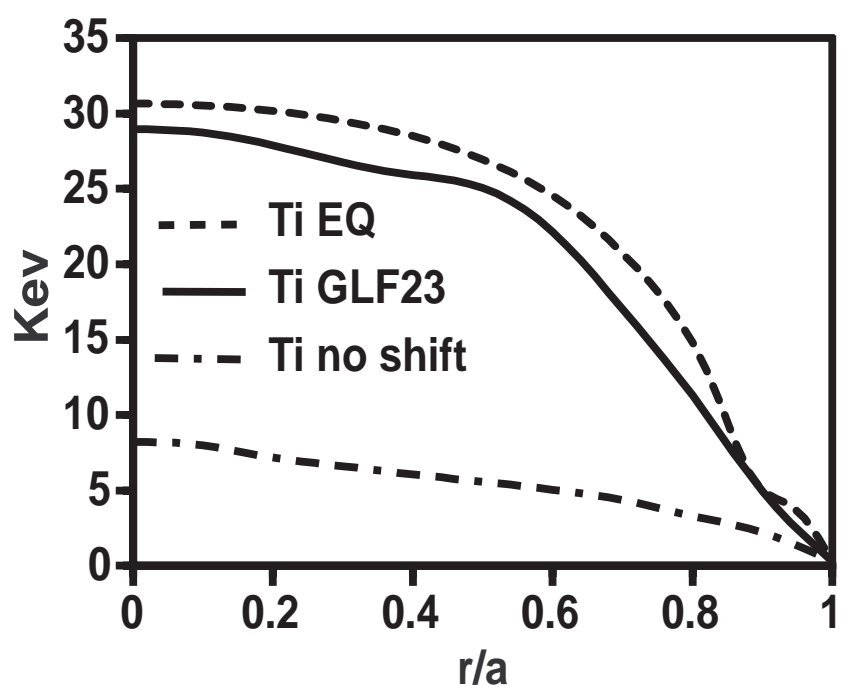

Figure 19: Case 1 ion temperature profiles used in the MHD equilibrium computed with the GLF23 model and computed with GLF23 with Shafranov shift effects turned off.

The first case is displayed in Figures 19, 20, 21, and 22. The ion (Fig. 19) and electron (Fig.20) temperatures are computed to be very close to the temperature profile used in the optimized MHD equilibrium. The density profile (Fig. 21) is peaked. Because the pressure profile is close to the high bootstrap fraction equilibrium the bootstrap current is well aligned with the total current density (Fig. 22). 


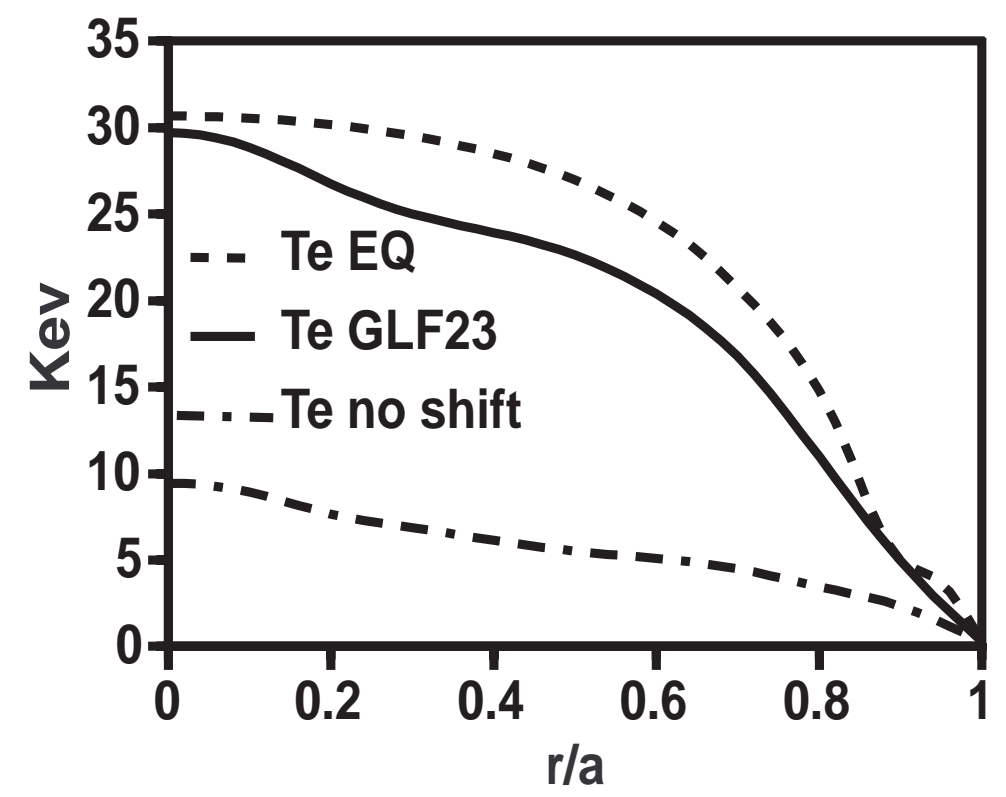

Figure 20: Case 1 electron temperature profiles used in the MHD equilibrium computed with the GLF23 model and computed with GLF23 with Shafranov shift effects turned off.

The effective ion thermal diffusivity is well above the Chang-Hinton neoclassical value over most of the profile, except very near the magnetic axis. Thus, there is no transport barrier with neoclassical ion thermal transport. In fact, the $E \times B$ velocity shear is small compared to the computed ITG growth so the plasma is not even close to a transport barrier threshold [40]. The third curve in Figures 19 and 20 are the computed temperature profiles without the Shafranov shift.

The Shafranov shift is the main cause of the improved transport in this case. The Shafranov shift causes an increase in the temperature gradient threshold for the drift waves but does not reduce the transport all the way to neoclassical. This has the advantage of giving improved transport without a localized steep gradient for MHD instabilities to feed off of. The fusion alpha particle heating for a $50 \%$ DT mixture was $610 \mathrm{MW}$. This had to be reduced to $370 \mathrm{MW}$ (with 53MW radiated) in order to be consistent with the ARIES-AT design values. This result is indeed promising but must not be taken at face value. The GLF23 model is based on shifted circle equilibria in the low beta approximation. These have a much weaker Shafranov shift than the ARIES-AT equilibrium. It is known that the GLF23 model overestimates the Shafranov shift stabilization for large values (MHD $\alpha>1$ ). The GLF23 calculations were also done in the electrostatic approximation. In the present calculations the Shafranov shift was multiplied by a factor 0.3. This was needed in order to avoid a complete suppression of the drift-wave transport (second stability). 
When all of the drift-wave transport is suppressed the only transport in the model for electrons is electron neoclassical. A runaway electron temperature thus occurs when all of the drift waves are suppressed. Note that $E \times B$ velocity shear generally cannot suppress ETG modes due to their high growth rates so the electron transport remains above neoclassical in an $E \times B$ shear induced barrier. The Shafranov shift suppresses all drift waves but may not give a second stability regime for drift-waves, as it does for MHD ballooning modes.

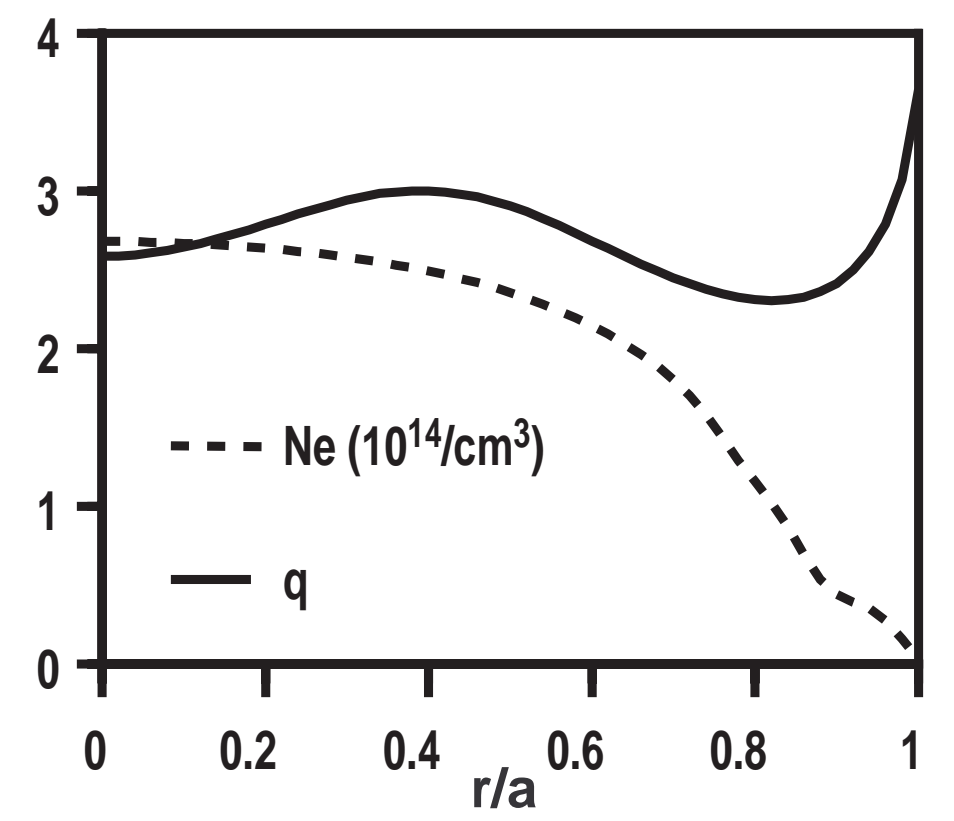

Figure 21: Case 1 equilibrium q-profile and electron density profile.

The suppression of drift wave growth rates for $\hat{S} \leq 0$ is well established experimentally. The GLF23 model may or may not be a faithful representation of this effect. This is the subject of present day research and has not yet been subjected to adequate experimental testing. Experiments on the physics of the Shafranov shift effect on turbulence in tokamaks are needed to test the theory. The peaking of the electron temperature near the magnetic axis in Figure 20 is the cause of the excess bootstrap current in this region (Figure 22). Experience with negative magnetic shear discharges in various tokamaks has shown a strong flattening of the electron temperature near the axis. The cause of this excess transport is unknown but it has been shown to not be due to linear drift-wave instabilities [41].

The second case is shown in Figures 23, 24, 25, and 26. This case used a modified MHD equilibrium with a non-zero current density at the separatrix. This has little effect on the transport calculations. The density profile Figure 25 was also taken to be broader than the first case. This change has a significant effect on the computed ion (a) and electron (b) temperature profiles. The pressure was below the target 


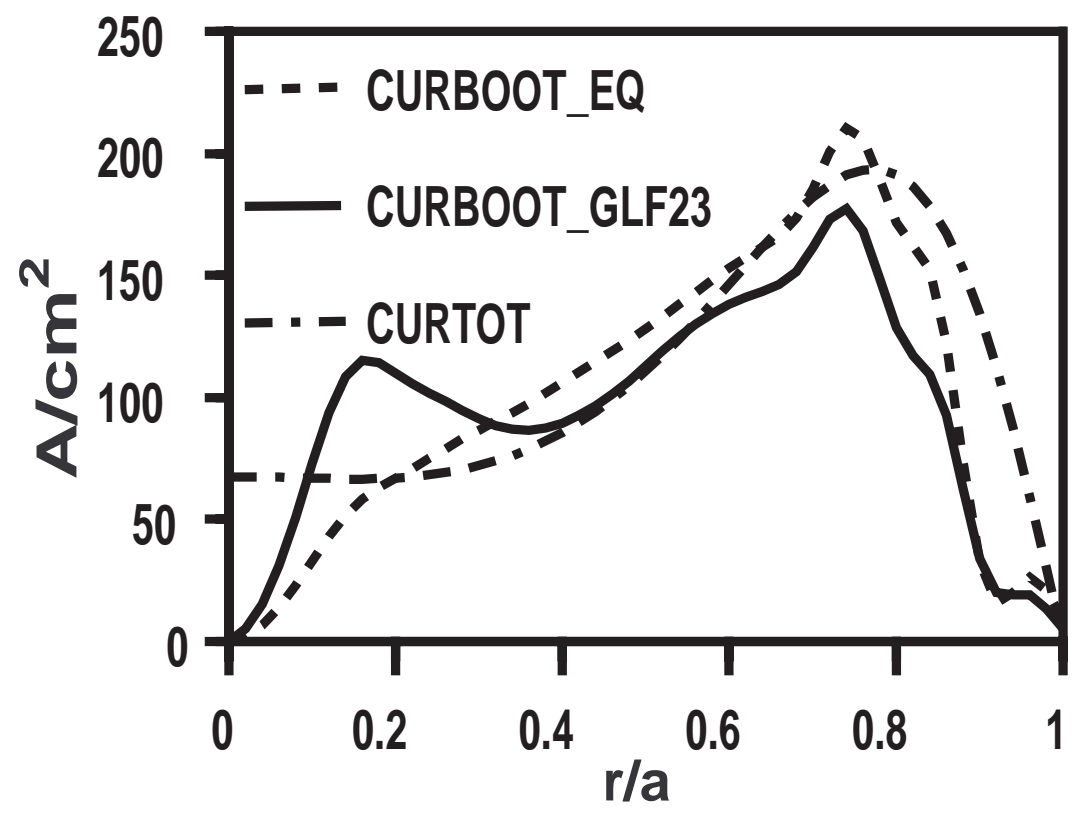

Figure 22: Case 1 equilibrium total current, bootstrap current for TOQ equilibrium and bootstrap current for GLF23 computed temperature profiles.

beta even with a $50 \%$ DT ion mixture producing $432 \mathrm{MW}$ of fusion alpha heating (70MW radiated). The bootstrap alignment was also degraded (Figure 26). This case has a boundary condition that the $E \times B$ velocity at the separatrix be equal to the value obtained from neoclassical theory just inside the separatrix. This is effectively a condition on the poloidal velocity. This boundary condition did not result in a significant $E \times B$ velocity shear at the separatrix. Thus, there is no transport barrier at the edge in this case.

The proper separatrix boundary condition is a delicate and complex issue. However, one can ask what the solution would look like for a case that is more like an $\mathrm{H}$-mode with an edge layer of suppressed transport. This case, Case 3, is shown in Figures $27,28,29$, and 30 . The $E \times B$ velocity was set to $-20 \mathrm{Km} / \mathrm{sec}$ at the separatrix for this case (whereas it was determined from the neoclassical value for the poloidal rotation for the first two cases). This produced significant $E \times B$ velocity shear not just at the separatrix but well into the plasma edge. The $E \times B$ velocity is not large enough to completely suppress the ITG mode but it does greatly reduce the edge transport by increasing the threshold temperature gradient for the ITG mode. The steepening of the edge temperatures is clearly seen comparing Figures 23 and 24 with Figures 27 and 28.

The edge density was also lowered in this case, Figure 29, but this did not by itself generate enough $E \times B$ shear. The large edge bootstrap current for this case is similar to that found for $\mathrm{H}$ and $\mathrm{VH}$-modes in present tokamaks. Experience 


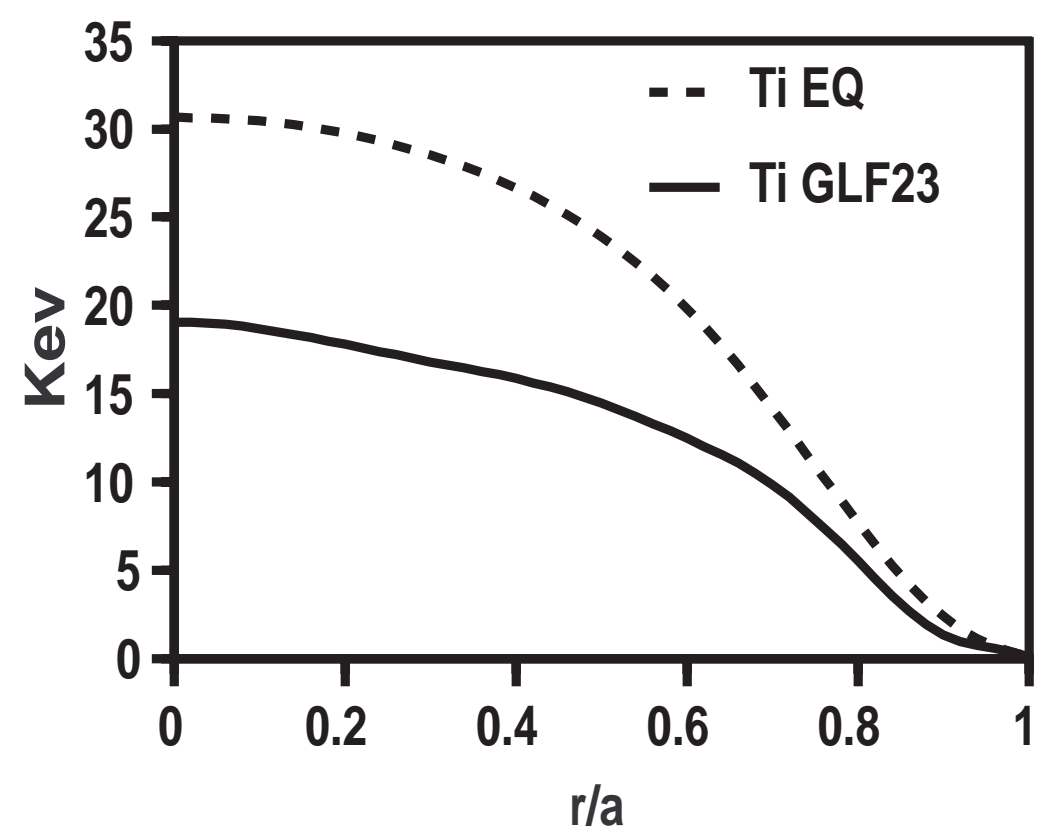

Figure 23: Case 2 ion temperature profiles used in the MHD equilibrium computed with the GLF23 model.

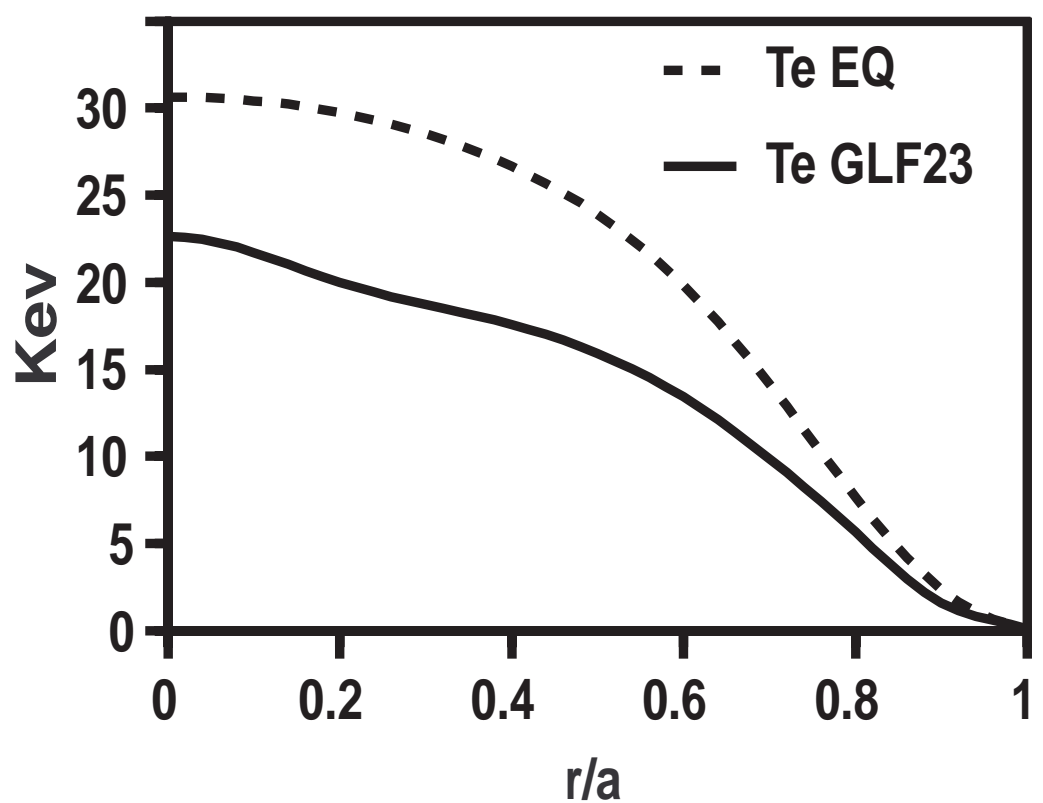

Figure 24: Case 2 electron temperature profiles used in the MHD equilibrium computed with the GLF23 model. 


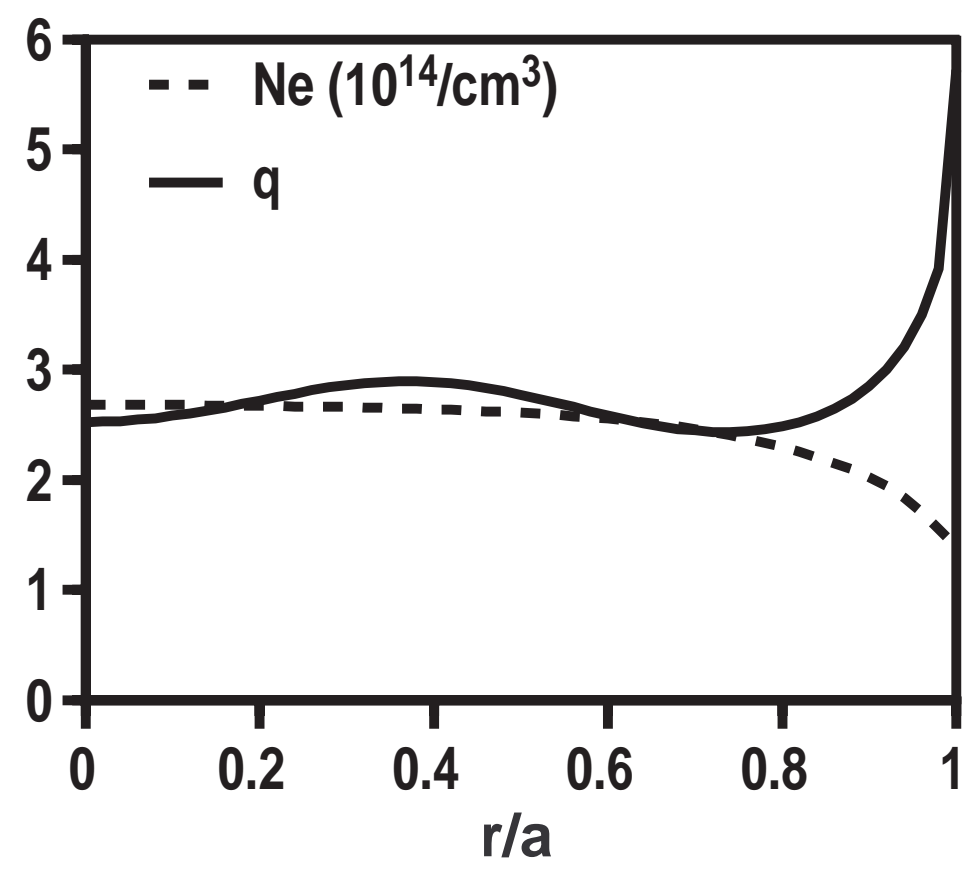

Figure 25: Case 2 equilibrium q-profile and electron density profile.

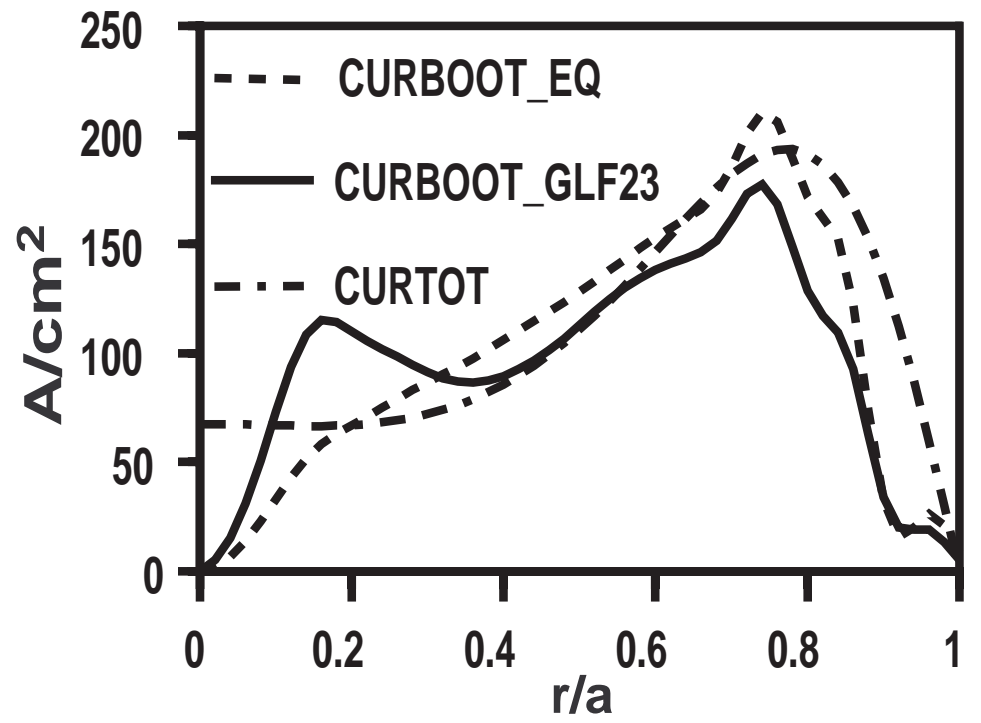

Figure 26: Case 2 equilibrium total current, bootstrap current for TOQ equilibrium and bootstrap current for GLF23 computed temperature profiles. 
with these regimes leads one to expect that this current profile would probably be unstable to edge kink modes. This is sometimes a benign condition, resulting only in edge localized modes which do not seriously degrade energy confinement. The $E \times B$ shear layer extends far from the edge more like a VH-mode in this case [42]. The transport is far too good for the size of this reactor design. The fusion power flow needed to sustain this beta was only $160 \mathrm{MW}$ (with $86 \mathrm{MW}$ ) radiated. Nevertheless, the ion thermal transport is still above neoclassical except near the axis. A redesign of the reactor would be required in order to take advantage of this regime.

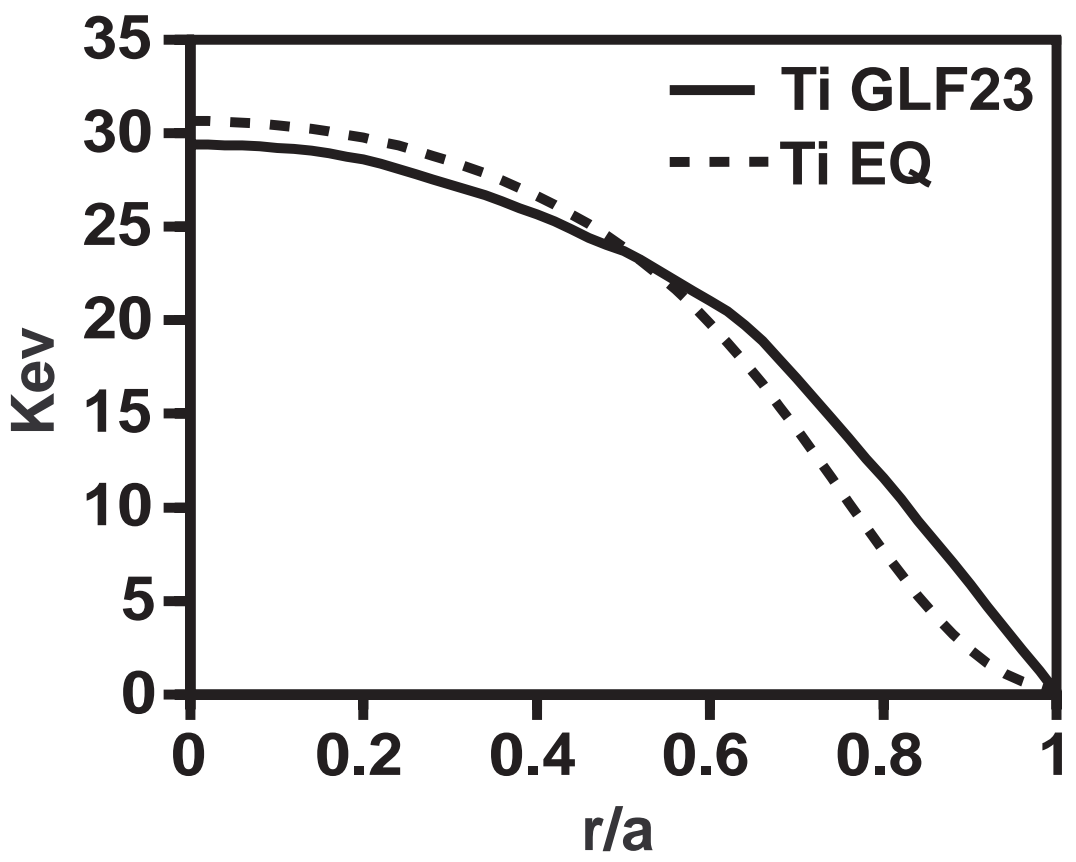

Figure 27: Case 3 ion temperature profiles used in the MHD equilibrium computed with the GLF23 model.

\subsubsection{Conclusions}

In summary, the GFL23 transport model does predict global energy confinement which exceeds the ARIES-AT design requirement if the density profile is peaked. The computed pressure profile is close to the one found for optimum MHD stability. The energy confinement improvement is primarily due to Shafranov shift stabilization of drift-waves. The turbulent transport is reduced but not completely suppressed by the large Shafranov shift. The theoretical basis of this effect is well founded. The exact level of transport suppression predicted by GLF23 is questionable however, since the Shafranov shift and beta are outside the range of the model's fit to theory. This work points to the need to try and produce plasmas in current tokamaks which have large Shafranov shifts without large $E \times B$ shear. Even in 


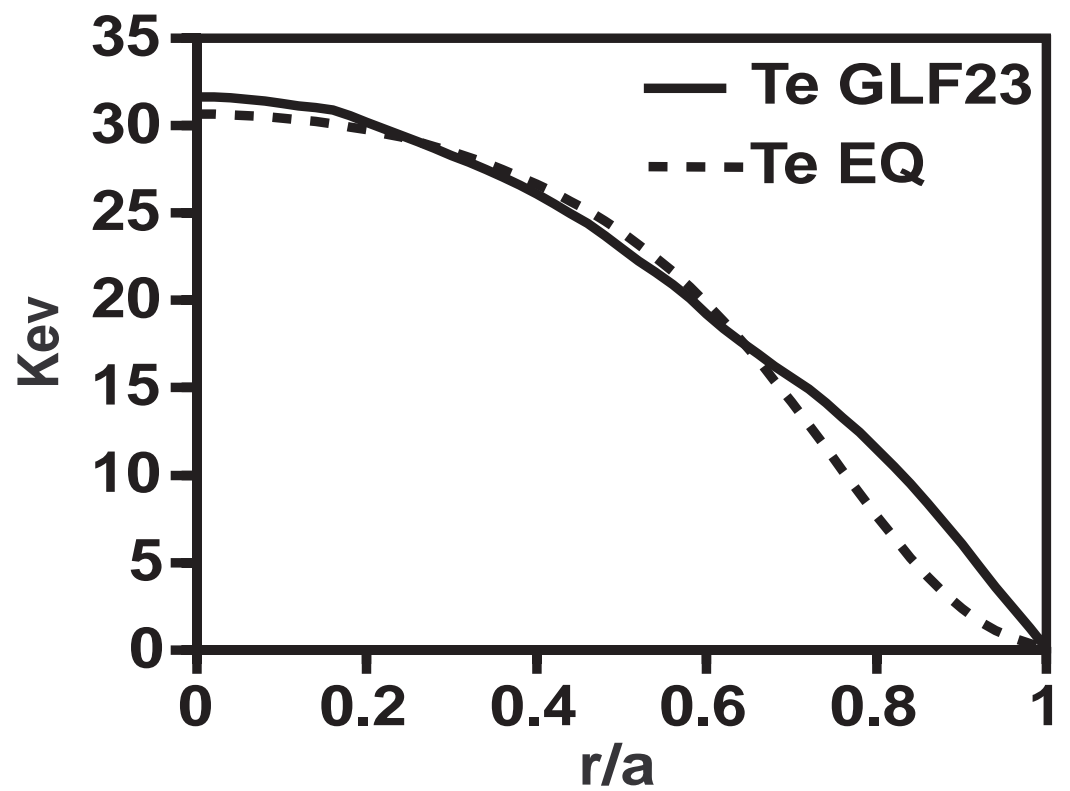

Figure 28: Case 3 electron temperature profiles used in the MHD equilibrium computed with the GLF23 model.

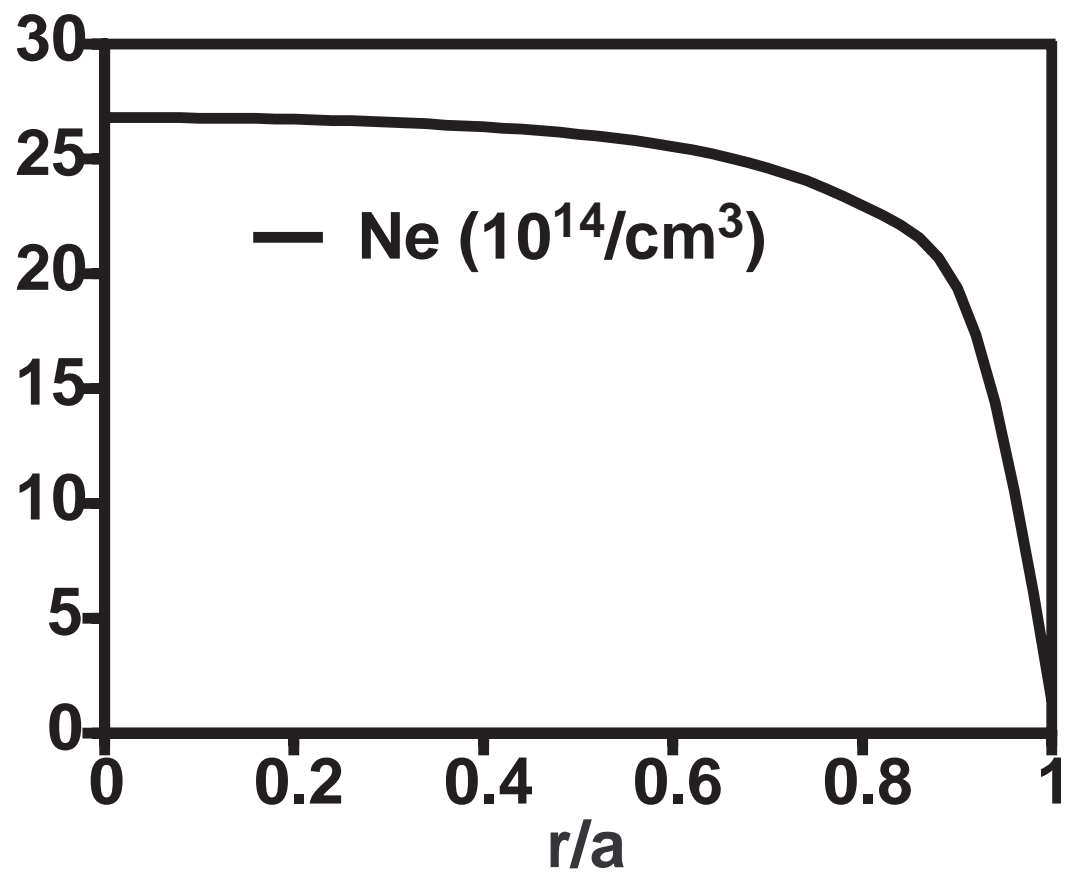

Figure 29: Case 3 electron density profile. 


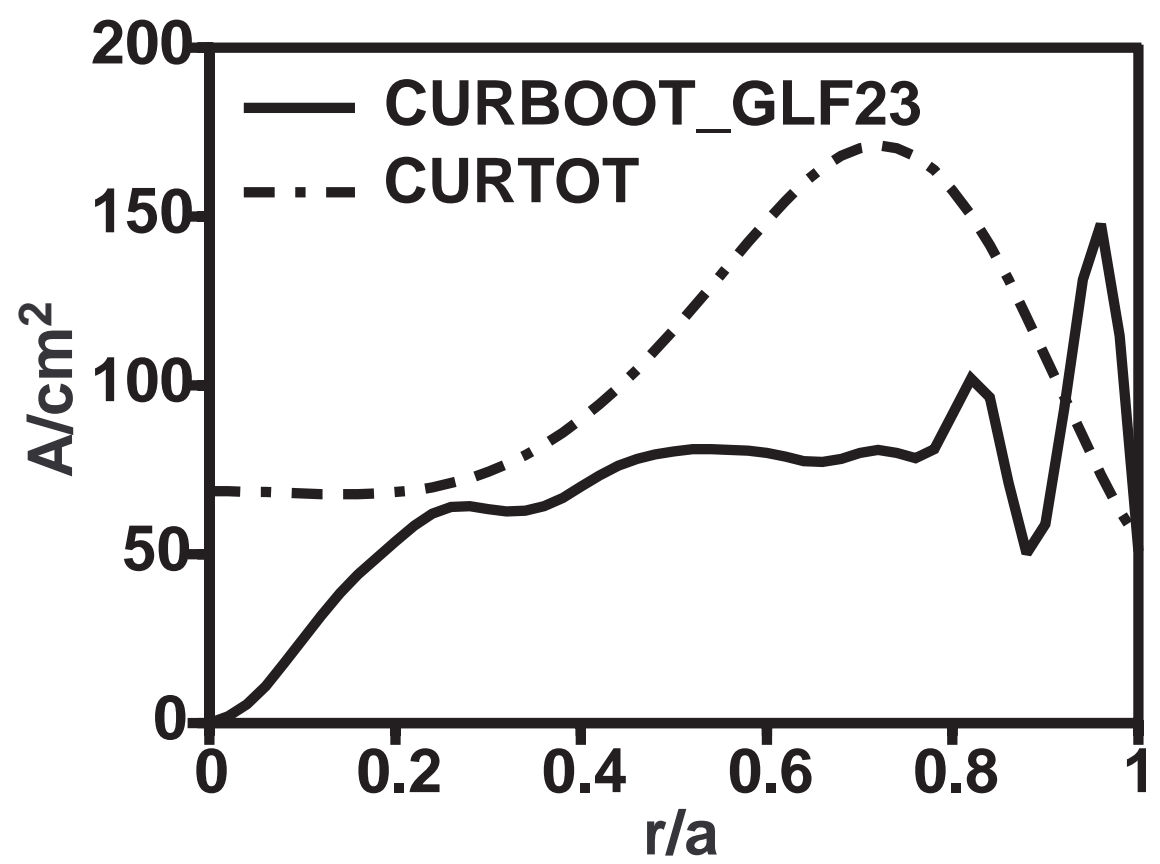

Figure 30: Case 3 equilibrium total current density and bootstrap current for GLF23 computed temperature profiles.

discharges with large enough $E \times B$ shear to quench ITG modes, the effect of the Shafranov shift on ETG modes could be studied.

Unexpectedly, the ARIES-AT plasma is quite unlike the better known regimes in current tokamaks, which owe their improved transport mostly due to $E \times B$ velocity shear and have relatively small Shafranov shifts. The high beta-poloidal regime [43] is the most similar to ARIES-AT. The requirement of high bootstrap fraction has pushed the design in this direction. If the density profile is broad with a sharp edge, the target energy confinement could only be reached with some help from $E \times B$ velocity shear. It is not possible to achieve sufficient toroidal rotation for transport improvement or RWM stability with a practical amount of neutral beam power. A method for changing the separatrix $E \times B$ velocity (say by differential puffing and pumping) could help induce an edge transport barrier. The GLF23 model predicts far too high an energy confinement for this case however and the bootstrap current is too far to the edge. This plasma is more like the VH-mode in present tokamaks.

\subsection{Toroidal Rotation Transport Study Using NBI}

The torque density due to $50 \mathrm{MW}$ of $120 \mathrm{Kev}$ neutral beams injected in the direction of the plasma current has been computed by ONETWO. The toroidal rotation due to this torque is computed using GLF23 in the XPTOR code. It has been estimated in Sec. 2.3 that an $E \times B$ rotation speed of at least several percent of the Alfven 
velocity, or about $500 \mathrm{~km} / \mathrm{sec}$ at the $q=3$ surface is needed to prevent the RWM for the L-mode edge design point (Case 1). The $q=3$ surface is very near to the edge $\rho=0.98$. This makes it impractical to achieve such a large rotation speed since the separatrix toroidal rotation is small and the rotation shear is limited by the large edge transport. The $E \times B$ velocity at the $q=3$ surface is predicted to be only $5 \mathrm{~km} / \mathrm{sec}$ for Case 1 . The toroidal rotation due to the $50 \mathrm{MW}$ of co-NBI was computed for this case but it made almost no difference at the $q=3$ surface. Very little shear is generated due to the large momentum diffusivity which for ITG modes is comparable to the ion thermal diffusivity. Thus we conclude that toroidal momentum injection by NBI is not a practical means of suppressing resistive wall modes in ARIES-AT.

\subsection{Estimate of Toroidal Rotation Driven by ICRF Minor- ity Heating}

Motivated by observations in Alcator C-Mod that plasmas heated by the minority ion-cyclotron heating can cause appreciable co-current toroidal rotation [44], a mechanism has been proposed by [45] to explain the results. This mechanism can drive rotation in tokamak plasmas even though the ICRH introduces negligible angular momentum. It has two elements: First, angular momentum transport is governed by a diffusion equation with a non-slip boundary condition at the plasma edge. Second, Monte-Carlo calculations show that energized particles will provide a torque density source which has a zero volume integral but separated positive and negative regions. With such a source the rotation frequency profile is given by

$$
\Omega(\phi)=\int d \phi \frac{T}{q V^{\prime}}<n M R^{2} \chi \ddot{U} \psi^{2}>
$$

where $T$ is the volume-integrated torque up to flux-surface $\phi$, and $\chi$ is the momentum diffusion coefficient. When the resonance is on the low-field side, the Monte-Carlo calculation predicts co-current rotation consistent with observation. The rotation becomes more counter and reverses sign for high-field side resonance according to simulation studies, but this has not been observed experimentally. Because of this, using this mechanism to control rotation should be considered speculative at present. Nevertheless, it is of interest to estimate the power requirement for ARIES-AT to produce adequate rotation to stabilize RWMs.

The power requirements for ARIES-AT for two cases corresponding to two different resonance locations (low-field side) are shown in Table 4.3. Figure 31 shows the rotation profile with the ICRF resonance at $r / a=0.34$.

In Sec. 2.3 we have estimated the rotation requirement for stabilization of RWM to be around 0.1 of the Alfven speed $V_{A}$. From our result and assuming that the energy confinement time $\tau_{E}$ is $\approx 1$ second, the ICRH power required would be $50-100$

MW. Finally, note that the effectiveness of this mechanism to produce rotational drive is significantly reduced when the resonant location is at $r / a>0.5$. 


\begin{tabular}{|l|l|l|}
\hline Resonance Location & $r / a=0.1$ & $r / a=0.34$ \\
\hline$V(0) / V_{A}$ & $2.1 \times 10^{-3}$ & $1.1 \times 10^{-3}$ \\
& $P(M W) \tau_{E}(S)$ & $P(M W) \tau_{E}(S)$ \\
\hline
\end{tabular}

Table 6: Power requirements for two different resonance locations.

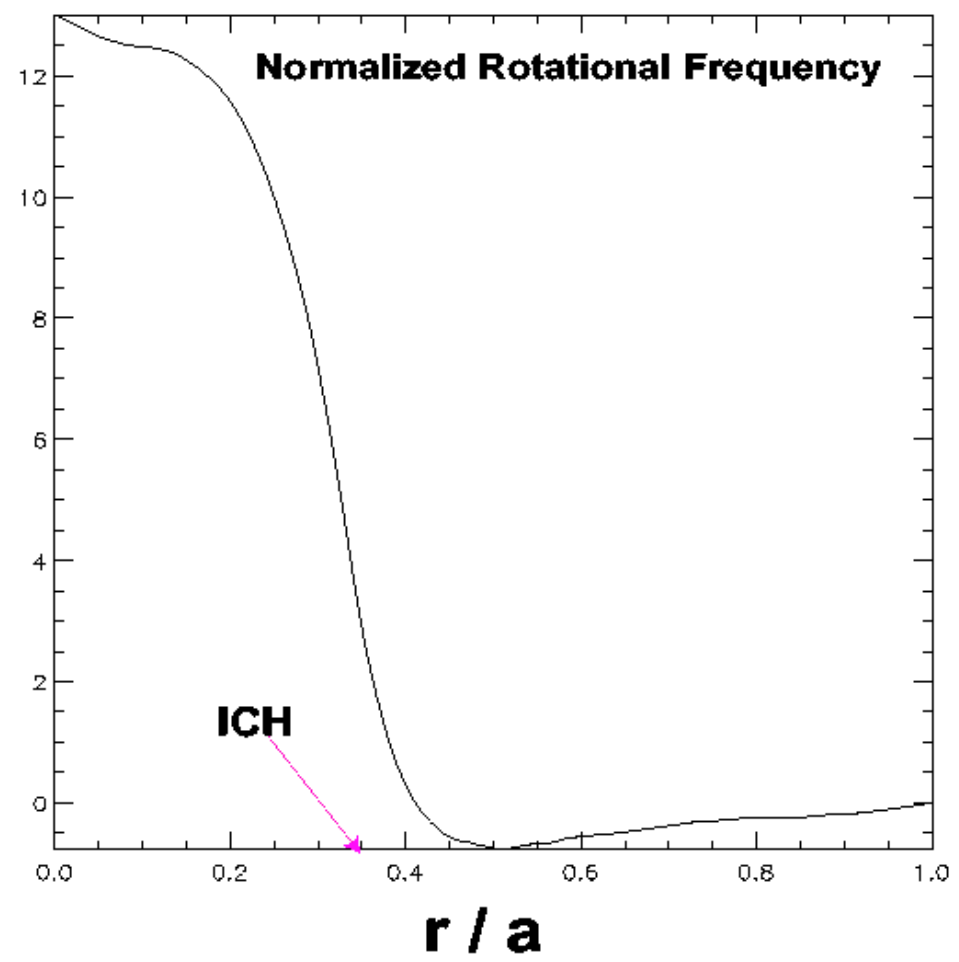

Figure 31: Rotation profile with the ICRF resonance at $r / a=0.34$ 


\section{Power and Particle Exhaust}

\subsection{General Considerations}

A major concern in the designing the ARIES-AT tokamak is excessive heat loading on its first wall from both electromagnetic (EM) radiated power and direct particle heating. In the following, we examine the intensity and poloidal variation of this heat loading, based on our present understanding of how these heat loading sources are distributed. Specifically, in Sections 5.1.1 and 5.1.2 we discuss the methodologies used in evaluating the radiative and particle contributions to total heat flux on the vessel surfaces. In Sec. 5.2 we use these methodologies to evaluate the heat flux and power loading under various operating scenarios. In Sec. 5.3 we review the efficacy of achieving the required radiated power from inside the core plasma, based on the most recent transport modeling.

\subsubsection{Calculating Radiated Power From Electromagnetic Sources}

An estimate of the poloidal radiated heat flux distribution along the first wall of the ARIES-AT vacuum vessel can be evaluated using the RADLOAD program. (The source and distribution of this radiated power is discussed in Sec. 5.3 of this report.) RADLOAD follows a "multi-filament" approach, where the incident power loading on a wall surface element $\left(Q_{E M}\right)$ from a radiating source distribution is given by:

$$
Q_{E M}=\int_{V} \epsilon_{R} \frac{\vec{r} \cdot \hat{n}}{4 \pi r^{3}} d V
$$

where $\epsilon_{R}$ is the radiating emissivity of the filament, $\vec{r}$ is the vector from the wall segment to the radiating filament, and $\hat{n}$ is the unit normal vector out of the wall surface element.

The RADLOAD program assumes toroidal symmetry. The above integral is performed numerically over that part of the radiating volume visible to a given surface wall element. To do this, the radiating region is divided into "filaments" extending around the machine in the toroidal direction with each filament denoted by its own set of poloidal coordinates. The limits of integration for each filament are determined by testing whether or not the line-of-sight along the filament is visible to a particular wall element. The heat flux $Q_{E M}$ is found by summing over the contribution of each filament.

Figure 32 divides the vessel wall into "segments" for which we will evaluate the radiated heat flux (both peak and average). For example, in order to estimate the spatial distribution of the radiating power source from inside the core plasma, we apply an "onion-ring" approach on the equilibrium shape shown in Figure 32. The plasma core is divided into four regions, each region lying between two specified flux surfaces and having (roughly) similar volumes. The total radiated power produced would be $\epsilon_{R}$ times the volume between any two (dashed) flux surfaces. To calculate the contribution of that ring to the incident radiated power flux for a specific poloidal 


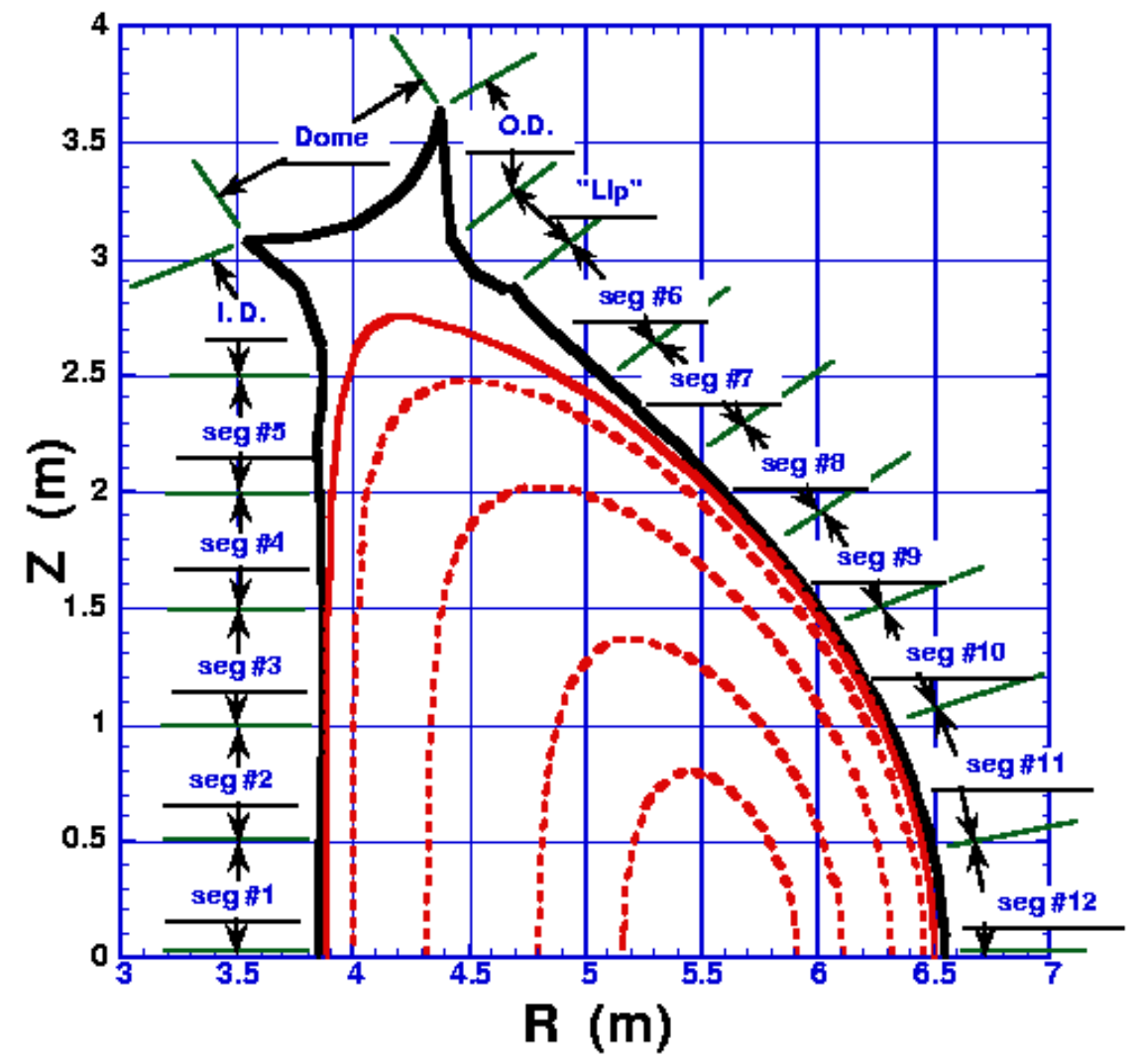

Figure 32: The poloidal locations of the wall segments are shown. Also shown are the flux surfaces used to define the "onion rings" needed in determining the radiating source function. 
location on the vessel wall, we assume that the power produced by the ring is distributed uniformly along a flux surface intermediate to the two flux surfaces which defines that specific plasma region. Typically, there are 200-300 radiating elements per region which must be individually evaluated. This method is repeated for each region.

\subsubsection{Calculating Particle Heating in the Divertors}

We assume that the radial profile of the heat flux in the scrape-off layer (SOL) has an exponential form. An expression for the peak heat flux at the divertor (along with "base case" values used in the calculation) can be written:

$$
Q_{\text {div }, S} \approx \frac{P_{\text {heat }} \times\left(1-f_{\text {rad }}\right) \times f_{\text {outboard } / \text { total }} \times f_{\nabla B / \text { total }} \times\left(1-f_{\text {pfr }}\right) \times \sin \alpha}{2 \pi \times R_{S} \times F_{\text {exp }} \times \lambda_{q_{\|}}}
$$

where $Q_{d i v, S}$ is the peak heat flux at the divertor strike point, $P_{\text {heat }}$ is the total heating power, $R_{S}$ is the major radius of the outer (inner) divertor strike point, $\lambda_{q_{\|}}$is the midplane parallel heat flux scrape-off width, $F_{\text {exp }}$ is the flux expansion at the outer (inner) divertor target, $\alpha$ is the angle between the divertor incline and the separatrix, $f_{\text {rad }}$ is the ratio of total radiated power to total input power, $f_{\text {outboard/total }}\left(f_{\text {inboard/total }}\right)$ is the ratio of power flowing into the outboard scrape-off layer (SOL) to the power flowing into the outboard (inboard) SOL, $f_{\nabla B / \text { total }}$ is the ratio of power striking the outboard divertor in the grad-B direction to the total power striking both the upper and the lower outboard divertors, and $f_{p f r}$ is the fraction of power flowing into the private flux region.

The peak heat flux predictions of Eq. (6) have been compared with DIII-D data and generally found to be within $20 \%$ of the measured peak heat flux at the outboard divertor strike point in attached plasmas. The values we use of the quantities in Eq. (6) are based on best estimates and extrapolations of available experimental data. For example: $\lambda_{q_{\mid l}}$ : A recent study [46] examined the ITER divertor power deposition database of L- and H-mode discharges and derived multi-machine scaling laws for heat flux widths based on machine geometry. L- mode scaling of the heat flux width, based on "measured divertor power" formulation [46], i.e.,

$$
\lambda_{q}(m)=6.6 \times 10^{-4} \times R(m)^{1.21} \times q_{95}^{0.59} \times \bar{n}_{e}^{0.54} \times Z_{e f f}^{0.61} \times P_{\text {div }}(M W)^{-0.19}
$$

can range from $\approx 1.4-2.1 \mathrm{~cm}$ using ARIES-AT parameters. However, $\lambda_{q}$ is defined by [46] as an "integral power width", instead of the width of the steepest part of the exponential decay nearest the separatrix, as we use in Eq. (6) above. We can convert $\lambda_{q}$ to $\lambda_{q_{||}}$by dividing Eq. (7) by 1.8. Thus, $\lambda_{q_{||}}$for our analysis is roughly $0.8-1.2$ $\mathrm{cm}$ in L-mode. For H-mode, the physics of the scrape-off layer is more complicated (e.g., ELMing) and Ref. [46] arrives at the expression:

$$
\lambda_{q}(m)=5.2 \times 10^{-3} \times P_{d i v}(M W)^{0.44} \times q_{95}^{0.57} \times B_{T}(T)^{-0.45}
$$


While Eq. (8) suggests that $\lambda_{q}$ increases with input power, this dependence is not a settled issue. For example, a recent analysis of power deposition profiles in JET has found that the power decay length may, in fact, decrease with power [47]. For this study, however, we use the results from [46], which was based on a multi-machine infrared thermography database. Inserting ARIES-AT parameters into Eq. (8), we find that $\lambda_{q} \approx 3.8 \mathrm{~cm}$, or $\lambda_{q_{||}} \approx 2.1 \mathrm{~cm}$. In summary, based on the most recent set of heat flux width scaling laws, we might estimate a $\lambda_{q_{||}}$range of roughly $0.8-2.1 \mathrm{~cm}$ for the L- and H-mode possibilities for ARIES-AT. The scaling laws for Eq.(7) and Eq.(8) were developed for single-null (SN) geometries. Recent studies on DIII-D, however, suggest that single-null and double-null (DN) $\lambda_{q_{||}}$are comparable, at least in H-mode [48].

$f_{\text {outboard/total }}$ : An estimate of this parameter can be obtained from simple geometric arguments by noting (1) that the ratio of the outboard plasma surface area to the inboard plasma surface area is $\approx 3: 1$ for ARIES-AT and (2) that the ratio of the radial temperature gradient on the outboard side to that of the inboard side of the plasma is $\approx 2.7: 1$. These imply that the ratio of the power flowing into the outboard SOL would be roughly eight times that flowing into the inboard SOL. Experimental data would support these arguments, where, for example, the ratio of the peak heat flux to the outer divertor target(s) to that of the inner divertor target(s) were found to be $\geq 8$ for high triangularity, DIII-D DN plasmas [49] [50]

$f_{p f r}$ : The power "spillover" into the private flux region has been observed on several tokamaks. For DIII-D, this value is typically 0.10-0.15.

$f_{\nabla B / \text { total }}$ : This value is sensitive to the precise magnetic balance near the DN configuration and thus ARIES-AT would require adequate feedback control over the magnetic balance between the divertors. The peak heat flux can be equalized if the $\mathrm{DN}$ is biased very slightly toward the direction opposite the $\nabla B$ ion drift [48]. In this study, we assume that a slight offset from "true" DN balance, such that $f_{\nabla B / \text { total }}=0.5$.

\subsection{Divertor and Wall Heat Loads}

In this section we apply the methods discussed above to possible operating scenarios for ARIES-AT.

\subsubsection{Application to the conservative case}

The parameters used in Eq. (6) are summarized in Table 7 and will be referred to as the "conservative" case parameters. For this, the peak heat flux under the inboard and outboard divertor legs is obtained by assuming that the radiated power from the scrape-off layer and divertors are negligible. This gives an upper bound estimate of the peak heat flux on the divertors, since including SOL and divertor radiated power would result in a reduced heat flux. The divertor legs in this design are inclined at an angle $(\alpha)$ of $10^{\circ}$ relative to the divertor surfaces in the slot in 
Table 7: "Conservative" Case Parameters

\begin{tabular}{|l|l|l|}
\hline & Inboard Leg(s) & Outboard Leg(s) \\
\hline$f_{\text {rad }}($ uniform distribution $)$ & 0.30 & 0.30 \\
\hline$\lambda_{q_{\|}}(\mathrm{cm})$ & 1.2 & 1.2 \\
\hline$F_{\text {exp }}$ & 2.5 & 4.0 \\
\hline$f_{p f r}$ & 0.1 & 0.1 \\
\hline$R_{s}(m)$ & 3.6 & 4.4 \\
\hline$f_{\text {outboard/total }}$ & 0.11 & 0.89 \\
\hline$f_{\nabla B / \text { total }}$ & 0.5 & 0.5 \\
\hline$\alpha\left({ }^{0}\right)$ & 10 & 10 \\
\hline$Q_{\text {div }, S}\left(M W / m^{2}\right)$ & 3.3 & 13.7 \\
\hline
\end{tabular}

order to increase the effective wetted area and minimize the heat flux. For this case, we further assume that $30 \%$ of the power is radiated (mostly bremsstrahlung) uniformly distributed inside the core plasma.

Figure 33 shows the peak heat flux for both inboard and outboard divertors as a function of $f_{\text {rad }}$ of the core plasma, assuming three different scrape-off power widths. For this study, the total heating power is $373 \mathrm{MW}$. If we take $\lambda_{q_{\| \mid}}=1.2 \mathrm{~cm}$ (which would be about right for an L-mode discharge and somewhat conservative for an H-mode discharge) and then use the "conservative" case parameters described in Table 7, we estimate $Q_{\text {div,peak }}$ for the inboard divertor ("I. D." segment in Figure 32 ) as $\approx 3.3 \mathrm{MW} / \mathrm{m}^{2}$ and for the outboard divertor ("O. D." segment in Figure 32) as $\approx 13.7 \mathrm{MW} / \mathrm{m}^{2}$.

The average heat fluxes on these inboard and outboard divertor segments are $\approx 0.8 \mathrm{MW} / \mathrm{m}^{2}$ and $\approx 7.1 \mathrm{MW} / \mathrm{m}^{2}$, respectively; the power flow "spillover" into the private flux region of the divertor produces an average particle heat flux of $\approx 0.5 \mathrm{MW} / \mathrm{m}^{2}$. The peak particle heat fluxes in the upper divertor are identical in their mirror locations in the lower divertor.

Figure 34 shows the poloidal distribution of the average (and peak) core-radiated heat flux (QEM) at each of the segments shown in Figure 32. While there is a pronounced poloidal distribution to the wall heat flux, their values are less than the $0.45 \mathrm{MW} / \mathrm{m}^{2}$ peak heat flux limit set by cooling concerns for non-divertor first wall locations.

What constitutes an "acceptable" peak divertor heat flux is problematical. ARIESAT engineering considerations presently set this limit at $\approx 5 \mathrm{MW} / \mathrm{m}^{2}$, which is much less than the $13.7 \mathrm{MW} / \mathrm{m}^{2}$ from our conservative estimate in this section. In the following section, we examine two less conservative scenarios that lower divertor heat flux values to more acceptable levels. 

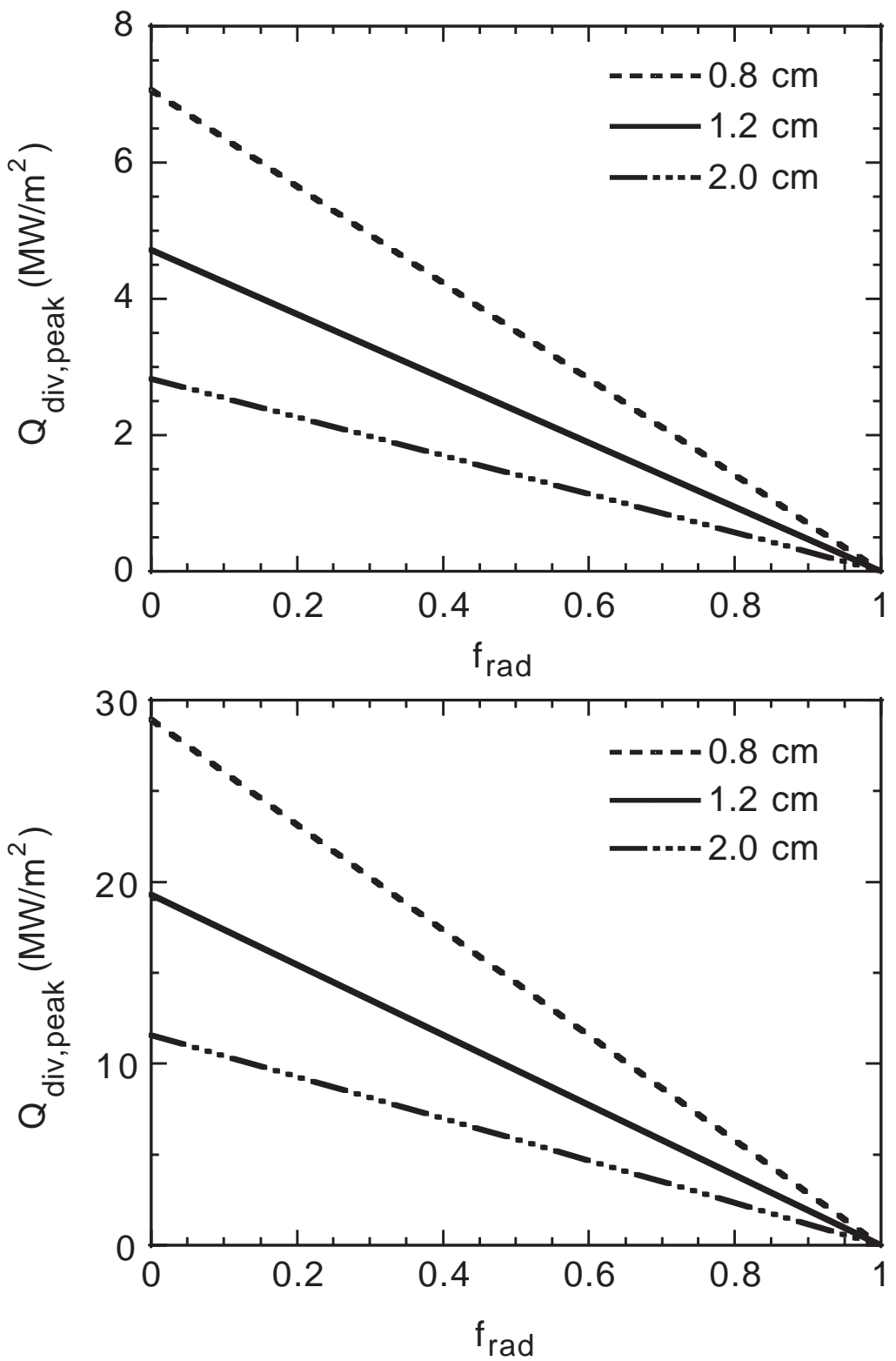

Figure 33: Estimates of the peak heat flux to the inboard (a) and outboard (b) divertors are shown as a function of the core radiated fraction, assuming three plausible parallel heat flux scrape-off widths. 


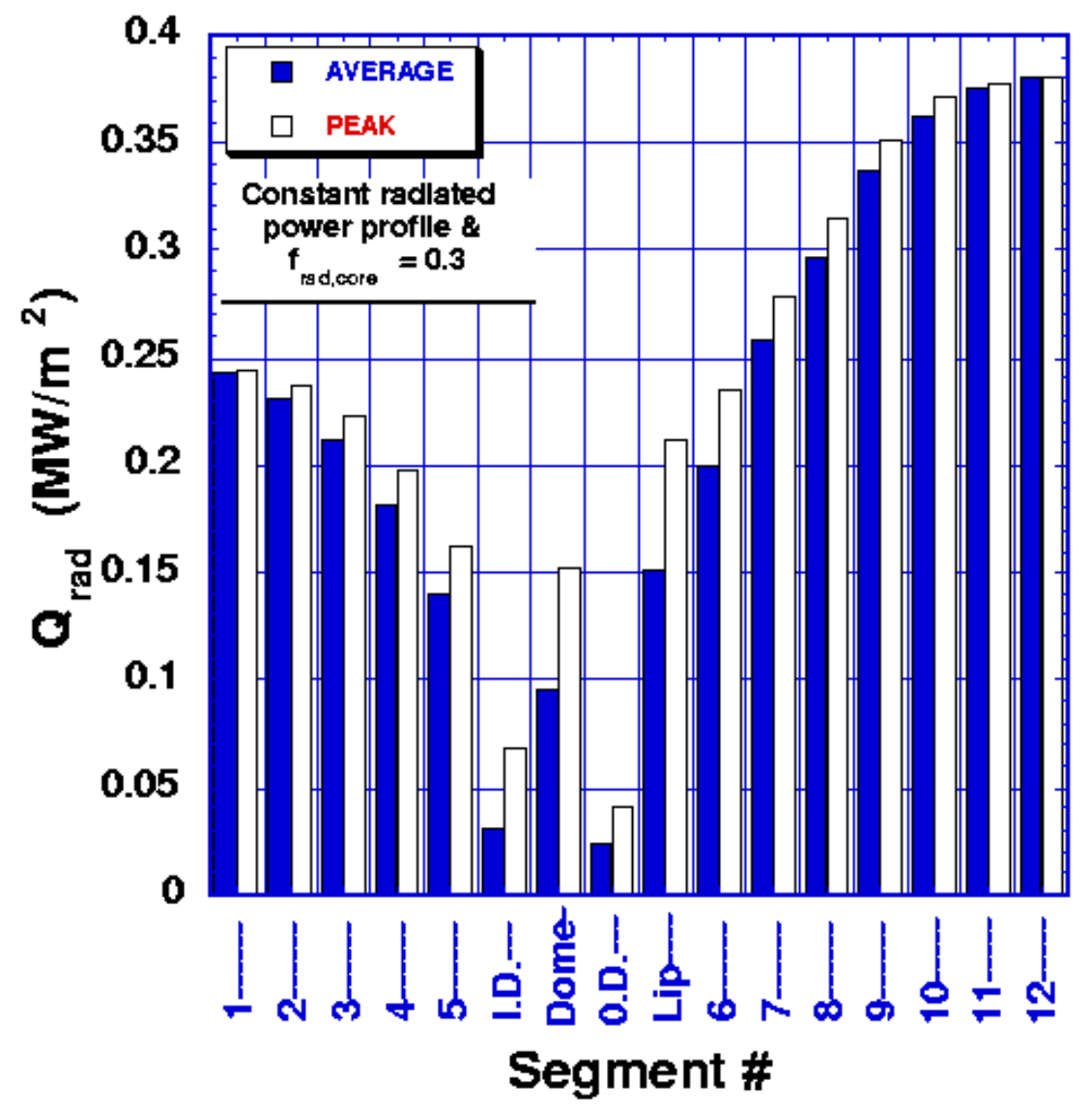

Figure 34: The poloidal distribution of the average and peak radiative heat fluxes are shown for the "conservative" case. No contribution from the divertor or scrape-off is assumed, in line with our conservative assumptions. The radiated power density is constant inside the core plasma 


\subsubsection{Radiating mantle approach}

Much of the physics in the boundary and SOL regions of single-null (SN) divertors, particularly the role of $E \times B$ poloidal drifts in the divertor [58, 59], has only recently come under the detailed scrutiny of transport modeling codes, such as UEDGE [60]. How these poloidal drifts affect asymmetries in divertor heat flux and particle flux [48] or the "enrichment" of radiating impurity ions in double-null (DN) divertors is speculative at the present time. Hence, it is prudent to first examine the efficacy of relying on the better-understood radiative processes occurring in the core plasma. Moreover, with radiating power from inside the core plasma, we not only avoid the complexities and uncertainties introduced by DN divertor physics, but also spread the plasma power more evenly around the vessel structure. From previous experience, we have found the "radiating mantle" concept effective in this regard, i.e., injected impurity ions radiate power predominantly from edge of the main plasma [51] [52] [53] [54].

Impurity injection has other advantages also. "Large" tokamaks, such as JET and JT-60U, have demonstrated that good energy confinement is consistent at higher densities under certain impurity injection scenarios [55] [56] [57]. The lower plasma edge temperatures which occur in a radiating mantle, particularly with the ion component, reduces the ion temperatures in the SOL, which in turn, reduces sputtering at the divertor plates from "hot" ions.

In this section we discuss one possibility based on the radiating mantle concept: the minimum radiated power for which the peak heat flux in the divertors is "manageable", i.e., $\approx 5 M W / \mathrm{m}^{2}$, under radiating mantle conditions. To do this,

we again take $\lambda_{q||}=0.012 \mathrm{~m}$ and $f_{\text {rad }} \approx 0.75$ (Figure 33), resulting in $Q_{d i v, S}$ to the outboard $\operatorname{leg}(\mathrm{s}) \approx 5 \mathrm{MW} / \mathrm{m}^{2}$ and to the inboard $\operatorname{leg}(\mathrm{s}) \approx 1.2 \mathrm{MW} / \mathrm{m}^{2}$; the average heat fluxes on these inboard ("I. D."), "dome", and outboard ("O. D.") segments are $\approx 0.3 M W / m^{2}, \approx 0.2 M W / m^{2}$, and $\approx 2.3 M W / m^{2}$, respectively.

The $75 \%$ core radiation requirement is about two and a half times that of the nominal case. In a parallel study using the MIST [64] impurity transport code to predict impurity behavior inside the core plasma a modest amount of argon impurities added to the plasma was successful in achieving this $75 \%$ radiated power requirement with a radiating mantle. (Details of impurity ion transport are discussed in Section 5.3. The resulting average and peak poloidal heat flux on the vessel walls are shown in Figure 35. At all poloidal locations, both the average and peak heat flux due to radiated power is $=0.9 \mathrm{MW} / \mathrm{m} 2$ with highest heat fluxes on the outboard vessel wall. Finally, as an upper bound estimate to wall heating by radiation, if it were possible to radiate all $373 \mathrm{MW}$ of power under a "mantle" scenario, we estimate that the highest radiative heat flux values would be $\leq 1.2 \mathrm{MW} / \mathrm{m}^{2}$ on the outboard wall.

Engineering considerations for cooling the (non-divertor) vacuum vessel wall of ARIES-AT, however, dictate that the average heat flux not exceed $0.3 \mathrm{MW} / \mathrm{m}^{2}$ (or, equivalently, that the peak heat flux not exceed $0.45 \mathrm{MW} / \mathrm{m}^{2}$ ). This implies that 


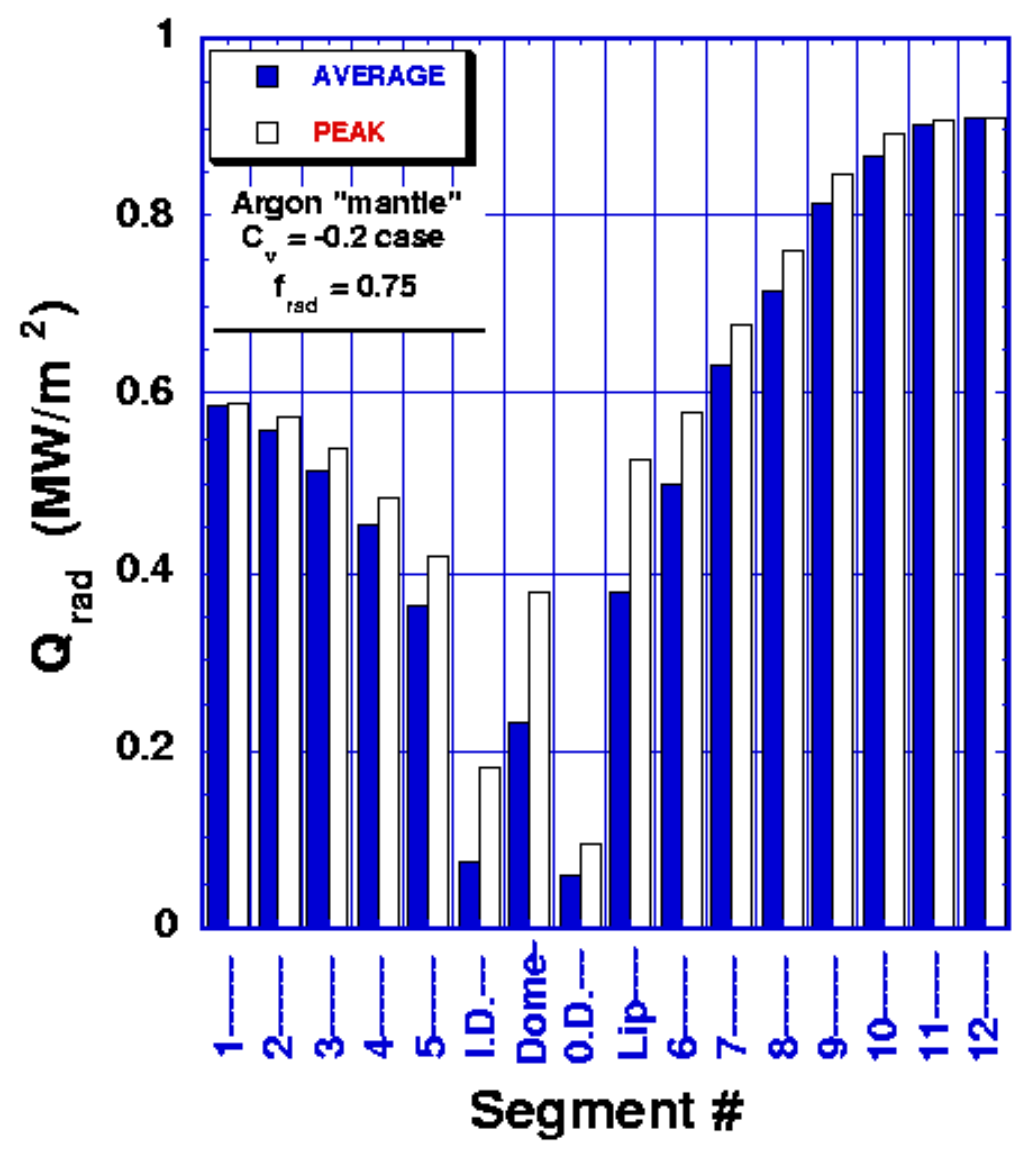

Figure 35: The poloidal distribution of the average and peak radiative heat fluxes are shown for the argon "mantle" case, as described in the text. As with the "nominal" case, no contribution from the divertor or scrape-off is assumed. 
the largest $f_{\text {rad }}$ of core radiated power which would not violate this engineering criterion is 0.36 . This limitation on the maximum radiated power from inside the core plasma means that the peak heat fluxes on the "I.D" and "O.D" segments can only be reduced to $3.0 \mathrm{MW} / \mathrm{m}^{2}$ and $12.5 \mathrm{MW} / \mathrm{m}^{2}$, respectively.

\subsubsection{Radiative divertor approach}

As discussed above, plasma edge/SOL transport codes, like UEDGE, are only now beginning to realistically address double-null divertor physics issues. However, it is still possible to get a handle on the amount of power that might be radiated away in the SOL and divertors. In a previous study of the ARIES-RS divertors, for example, a simplified radiative divertor approach provided a plausible solution to the divertor heat flux problem by seeding the divertor with a neon impurity, although this required several favorable assumptions in the divertor physics [2].

Here, we again look at this radiative divertor approach, but this time we choose argon as the seeding impurity. Like neon, argon can be expected to radiate reasonably well under ARIES-AT divertor and scrape-off layer conditions. Unlike neon, however, argon radiates much more efficiently in the edge region of the ARIES-AT plasma, enhancing radiative power from the core plasma mantle. In turn, a radiating plasma mantle relaxes the "enrichment" requirement (i.e., the ratio of $n_{e A R} / n_{e}$ in the divertor to that in the core) for the radiating impurity in the divertor. In order to reduce the total (particle plus radiative) peak heat flux in the divertors to $\approx 5-6 M W / m^{2}$, we require $\approx 160 M W$ of radiated power in the scrape-off layer and divertors (i.e., $f_{\text {rad,div }} \approx 0.4$ ), in addition to the core radiative fraction of 0.36 . Note: this implies that $Q_{\text {div,peak }}=4.0 \mathrm{MW} / \mathrm{m}^{2}$ in an attached plasma case; choosing this value to be well under the $5 \mathrm{MW} / \mathrm{m}^{2}$ limit is necessary, since there will be a contribution to the heat flux from the radiated power in the divertors.

As an initial estimate of radiated power in the divertor, we use a simplified UEDGE simulation [61] of an ARIES-AT-like edge plasma case. Among the simplifying assumptions are: (1) up/down symmetric solutions in the upper and lower divertors, (2) no $\nabla B$ or poloidal $E \times B$ drifts included in this modeling, (3) an orthogonal coordinate system, i.e., the flux surfaces intersect the divertor structure at normal incidence, and (4) inboard and outboard strike points are isolated from each other, e.g., what occurs at the inboard divertor does not affect the outboard divertor.

The $n_{e}\left(n_{e, s e p}=0.5 \times 10^{20} \mathrm{~m}^{-3}\right)$ and $T_{e}\left(T_{e, s e p}=340 \mathrm{eV}\right)$ radial profiles in the scrape-off and divertor regions are determined self-consistently for a hydrogenic ARIES-AT plasma, where the electron and ion diffusivities are $0.5 \mathrm{~m}^{2} / \mathrm{s}$ and the particle diffusivity is $0.33 \mathrm{~m}^{2} / \mathrm{s}$. We then estimate the radiated power from the argon present by assuming a fixed-fraction of argon in the SOL/divertor $\left(n_{e A R} / n_{e} \approx\right.$ $0.0026)$, which, in turn, produces $\approx 160 M W$ of radiated power in the SOL/divertors. Under these restrictions, virtually all the radiated power occurs between the divertor tiles and the respective X-points, particularly nearest the divertor floor (ceiling). We 
should point out that $n_{e A R} / n_{e} \approx 0.0026$ does not necessarily mean that this ratio will also be the concentration of argon in the core plasma. For example, recent "Puff and Pump" experiments in DIII-D single-null diverted plasmas have shown that an enrichment of $\approx 3$ is possible, even when a significant amount of argon is injected [62]. Interestingly, the measured fraction $n_{e A R} / n_{e}(\approx 0.0035)$ in the divertor is comparable with our estimated requirement for ARIES-AT.

For the next step of complexity, we wanted to allow UEDGE to seek a more selfconsistent solution, i.e., fix the ratio $n_{e A R} / n_{e}$ in the scrape-off layer and divertor and then find the self-consistent solution in the SOL and divertor. Unfortunately, it is more difficult to obtain self-consistent fixed-fraction solutions because numerical issues such as grid resolution and possible unstable plasma behavior, e.g., MARFE's, can arise for strongly radiating detached divertor plasmas. These simulations require more manpower and computational resources for a solution. The most realistic simulation of radiating impurities in UEDGE would use the multi-charge-state model and compute the self-consistent spatial distribution of the impurities as a result of plasma transport and atomic processes. Such simulations have been carried out for the ITER reference design [63]. These solutions are not available at this time. We believe, however, that detachment of both the inboard and outboard divertor legs along the separatrix would occur for argon fractions of only a few tenths of a percent.

From engineering considerations, the peak heat flux should not exceed $0.45 \mathrm{MW} / \mathrm{m}^{2}$ on the (non-divertor) vacuum vessel surfaces and should be $\approx 5 \mathrm{MW} / \mathrm{m}^{2}$ on the divertor wall segments. In the likelihood that both inboard and outboard separatrix strike points are detached, the peaked heat flux formulas used in Eq. (6) overestimates the actual peak heat flux. Hence, under detached conditions, our calculation of the total peaked heat flux (below) would be overestimates.

In order to evaluate the contribution of the radiated power from the ARIES-AT divertors to wall heating, we take the radiating divertor source to be distributed uniformly under the X-points between the separatrix flux surface and the flux surface 1-cm outside the separatrix (as measured from the midplane). Figure 36 shows the combined poloidal distribution of the radiated power from the core plasma $\left(f_{\text {rad,core }} \approx 0.36\right.$ and divertor plasma $\left(f_{\text {rad,div }} \approx 0.43\right)$, as determined from RADLOAD. The total heat flux on any element of the vacuum vessel wall is the sum of the radiative $\left(Q_{\text {rad }}\right)$ and the particle $\left(Q_{\text {div }}\right)$ contributions. The "worst case" scenario occurs when the peak in the radiative contribution and particle flux contribution coincide on the "O.D." segment. Under such a conservative scenario, the sum of the peak radiative heat flux contribution to the "O.D" segment $\left(\approx 1.8 \mathrm{MW} / \mathrm{m}^{2}\right.$ in Figure 32) and particle heat flux contribution $\left(\approx 4.0 \mathrm{MW} / \mathrm{m}^{2}\right)$ is roughly $5.8 \mathrm{MW} / \mathrm{m}^{2}$.

\subsubsection{Summary and Conclusion}

We considered reducing high peak heat flux at the divertors by radiating away power from inside the core plasma and/or in the divertors. First, in order to minimize over 


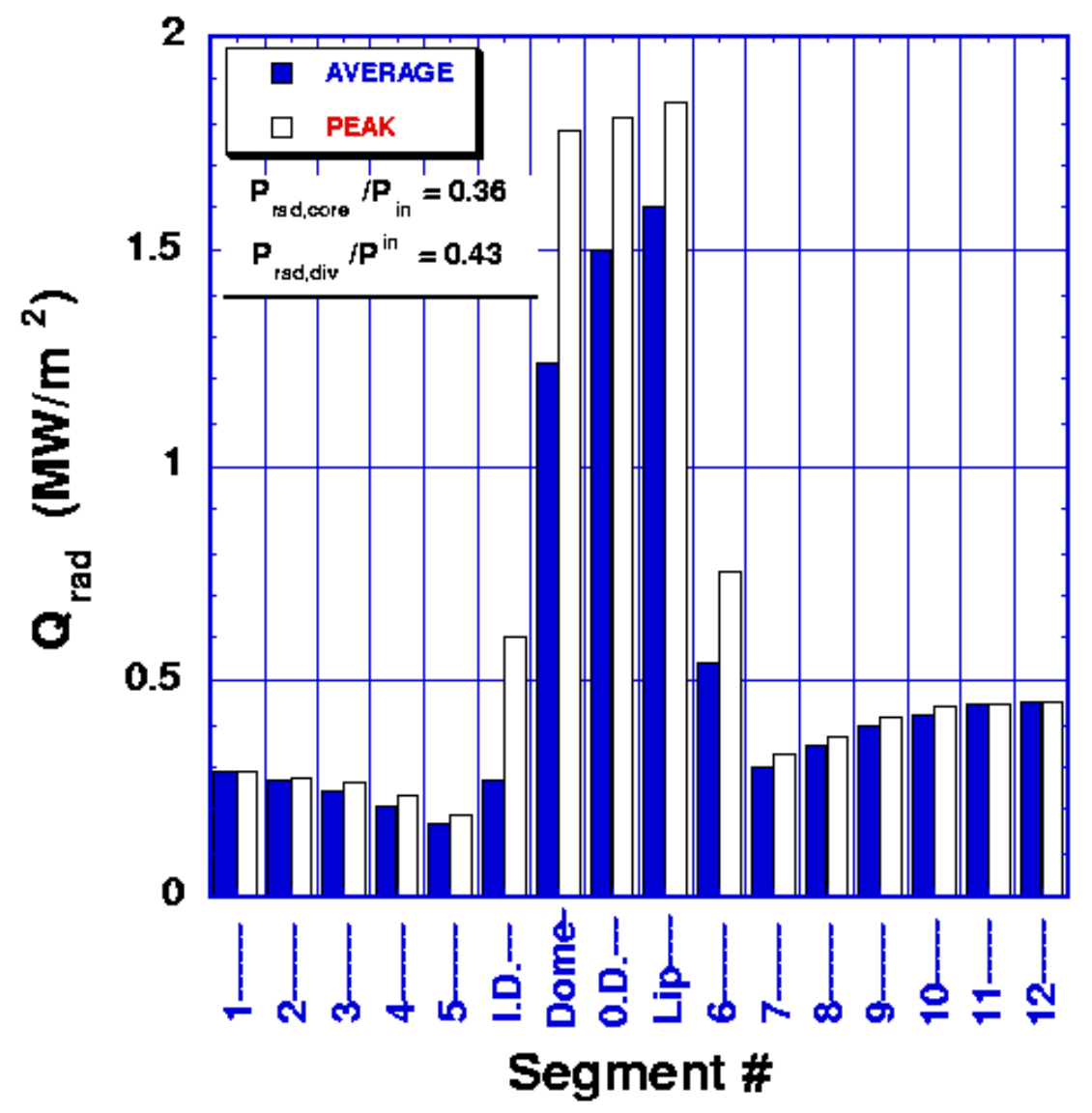

Figure 36: The poloidal distribution of the average and peak radiative heat fluxes are shown for the argon "radiative divertor" case, as described in the text. Thirty six percent of the power input is radiated in the core and $43 \%$ of the power input is radiated in the divertor. Outside the divertor, the radiative heat flux to the vessel walls does not exceed $0.45 \mathrm{MW} / \mathrm{m}^{2}$. 
reliance on several speculative and largely unexplored aspects of DN divertor physics (e.g., poloidal drifts and impurity entrainment in the SOL and divertor), we took a conservative approach of ignoring any radiated power from the SOL and divertor region while concentrating on core radiated power. Although the radiating away the power inside the core plasma has the advantage of dissipating the power more evenly on the vessel wall, wall-cooling considerations in present ARIES-AT design restricted the usefulness of this approach to a relatively low radiating fraction, i.e., $f_{\text {rad,core }}=0.36$. (In this regard, future work in raising the cooling capacity of the non-divertor vacuum vessel walls in order to allow a higher $f_{\text {rad,core }}$ should be considered.) In order to reduce the peak divertor heat flux to the $5-6 \mathrm{MW} / \mathrm{m}^{2}$ range, we were required to radiate about $40 \%$ of the heating power in the divertor, i.e., $f_{\text {rad,div }} \approx 0.4$.

\subsection{Core Plasma Impurity Radiation in ARIES-AT: L-mode Edge Case}

\subsubsection{Overview}

The power originating in the core plasma, i.e. the sum of fusion alpha particle power and auxiliary heating and current drive power, is exhausted as electromagnetic radiation from the core plasma or as particle kinetic energy transported across the separatrix to the scrape off layer and then along the open field lines to the divertor. To alleviate the thermal loads on the divertor surfaces in contact with the open field lines, it is desirable to radiate power from the core plasma. Bremsstrahlung and synchrotron radiation arises naturally from the core plasma. Bremsstrahlung can be enhanced by the injection of rare gas impurities. Impurities will also radiate due to plasma excitation of line radiation. In this section we discuss the bremsstrahlung and impurity line radiation induced by rare gas impurities intentionally injected into the core plasma. The engineering constraints due to the ARIES-AT main chamber first wall thermal design limit the total core radiation to about $140 \mathrm{MW}$. The estimated synchrotron radiation loss to the first wall is about $18 \mathrm{MW}$, leaving about $120 \mathrm{MW}$ of desired impurity radiation. We will show calculations of $\mathrm{Ne}$, Ar, and $\mathrm{Kr}$ radiation for the L-mode edge case of ARIES-AT using anomalous transport and a case for Ar using neoclassical transport. In these cases, the bremsstrahlung radiation alone is sufficient to produce the required $120 \mathrm{MW}$. An even higher level of radiation can be achieved from $\mathrm{Ar}$ and $\mathrm{Kr}$ due to additional line radiation.

\subsubsection{Anomalous Transport Modeling Using MIST}

The MIST model [64] has been used to estimate the impurity radiation induced using injected rare gases. The MIST code is a 1D trace impurity transport model. Steady state impurity density profiles, and the resulting radiation profiles are determined for given electron temperature and density profiles and transport coefficients. This 
version of the MIST code assumes a circular cross section plasma. However, when the total radiation is calculated from the modeled radiation profile, the actual plasma shape from the EFIT solution [3] is used.

The electron density profile being considered has an exponential fall-off beyond the separatrix. The central electron density is determined from the total density, $6.306 \times 10^{20} \mathrm{~m}^{-3}$, correcting for the impurity ion fractions, e.g. $f_{N e}=0.0075$. For these calculations the presence of helium has been ignored. The impact is small, only a slight reduction in the calculated bremsstrahlung radiation from the background plasma. The electron density profile is shown in Figure 37.

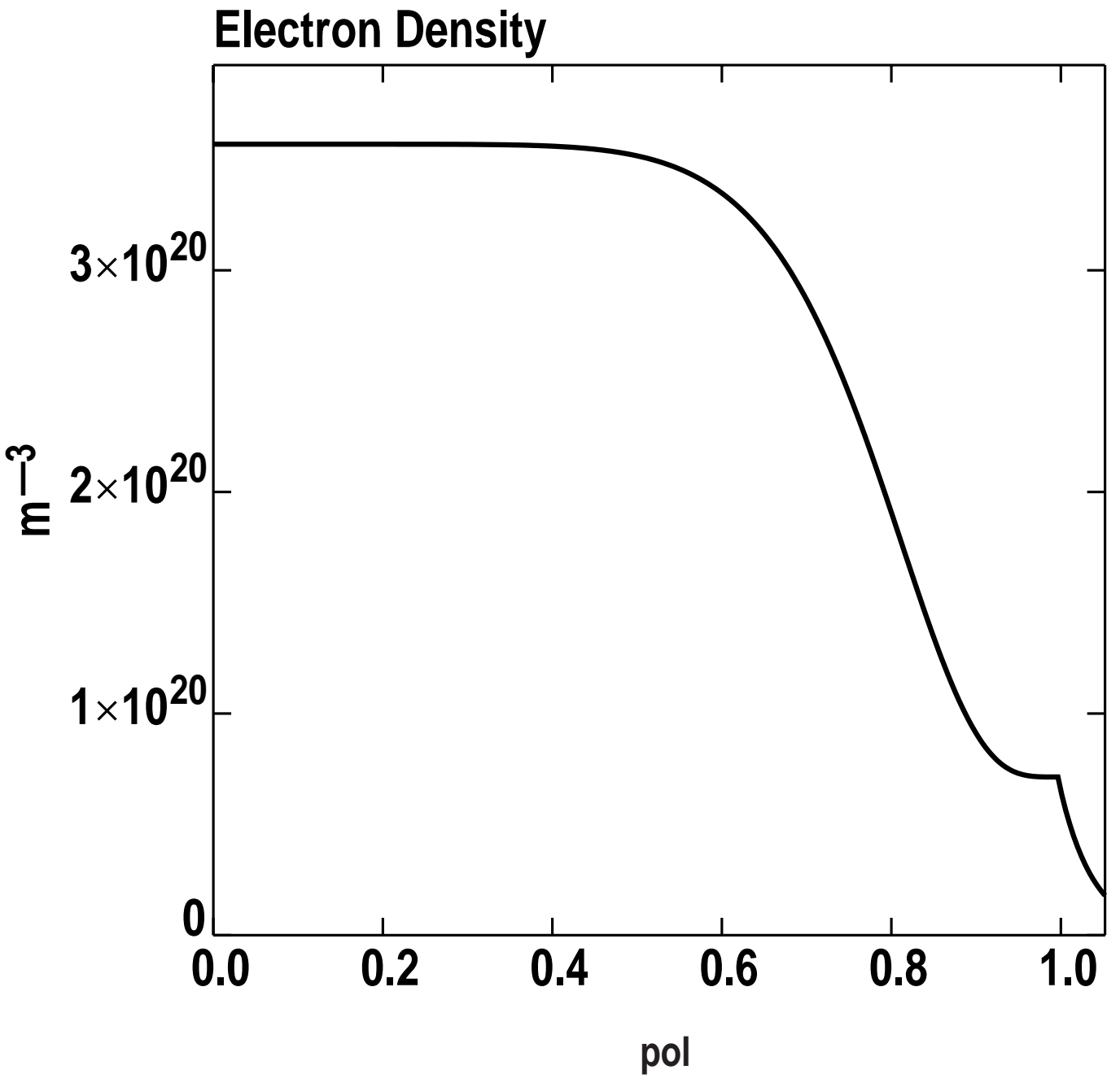

Figure 37: The ARIES L-mode edge density profile used in the core impurity radiation study.

The electron temperature profile, shown in Figure 38, is obtained from the EFIT pressure profile and the total density profile (same shape as the electron density profile with $n_{0}=6.306 \times 10^{20} \mathrm{~m}^{-3}$ ). The EFIT pressure profile goes to zero at the 
separatrix. To allow for a finite SOL temperature, a constant temperature of 300 $\mathrm{eV}$ is added to the $T_{e}$ profile for $\rho<1.0$, with an exponential fall off for $\rho>1.0$.

In this study we have used a constant impurity fraction as a boundary condition at the central axis. For neon, we have chosen the fraction of 0.0075 assumed in the systems study. For argon and krypton, the central fractions used produced a somewhat higher radiated power yet lower core $Z_{\text {eff }}$ and core dilution.

We have also chosen to use a spatially constant particle diffusion coefficient, $D=10^{4} \mathrm{~cm}^{2} / \mathrm{s}$. Because we are using a fixed central impurity fraction, and using steady state solutions, the calculated radiation profiles are only weakly dependent on the value of D. The impurity density profile shape does significantly effect the calculated total radiation. For the MIST modeling we have used the $C_{V}$ coefficient to set the particle pinch velocity,

$$
v_{p}=C_{V}\left(D / n_{e}\right) \frac{d n_{e}}{d r}
$$

where $v_{p}$ is the local particle convective velocity. For $C_{V}=1.0$, the impurity density profile has the same shape as the electron density profile. The values of $D=$ $10^{4} \mathrm{~cm}^{2} / \mathrm{s}$ and $C_{V}=1.0$ are consistent with measurements on DIII-D in L-mode plasmas when anomalous transport dominates.

In Figure 39 radial profiles of impurity density, volume emissivity for all ions, $Z_{\text {eff }}$, and volume integrated power using argon with a $C_{V}=1.0$ are shown. The radiation includes both the line and bremsstrahlung radiation from each ionization state. Since Ar is fully stripped over most of the volume of the confined plasma, the bulk of the radiation is due to bremsstrahlung. The solid line in Fig. 39 (d) is bremsstrahlung and line radiation from argon, the dashed line is bremsstrahlung from the primary deuterium ions.

Figure 40 shows the radial profiles of krypton ion density and volume integrated power using krypton with a $C_{V}=1.0$. The central $Z_{\text {eff }}$ for $\mathrm{Kr}$ has been reduced to 1.5 (compared to 1.6 for Ar), and fuel dilution is also lower, yet the total radiated power fraction is higher, about 0.5. This is due to the fact that krypton is not fully ionized in the ARIES-AT plasma, and therefore produces more line radiation.

\subsubsection{Neoclassical Impurity Transport Modeling using STRAHL}

Inside the transport barrier in ARIES-AT we expect that anomalous transport should be reduced to very low levels and neoclassical transport should dominate. Impurity transport measurements on DIII-D have indicated that neoclassical effects are important during enhanced confinement operation [67]. We have modeled neoclassical transport for argon in the ARIES-AT L-mode edge plasma using the STRAHL code $[65,66]$.

The resulting effective transport coefficients are shown in Figure 41. The neoclassical pinch velocity as computed by the STRAHL code is seen to be outward in the central region of the plasma. This outward pinch results from the nearly 


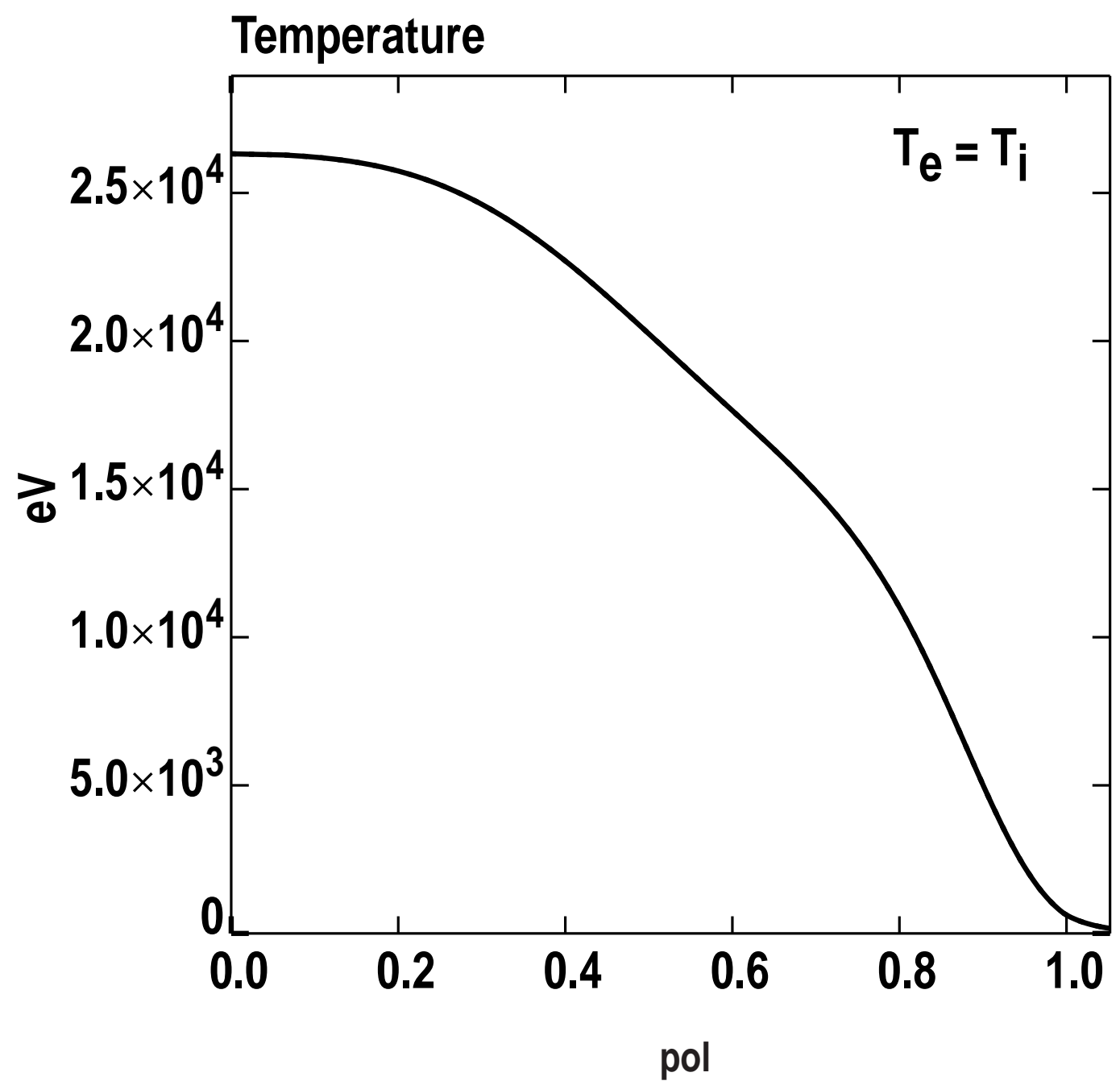

Figure 38: The ARIES L-mode edge ion and electron temperature profile used in the core impurity radiation study. 

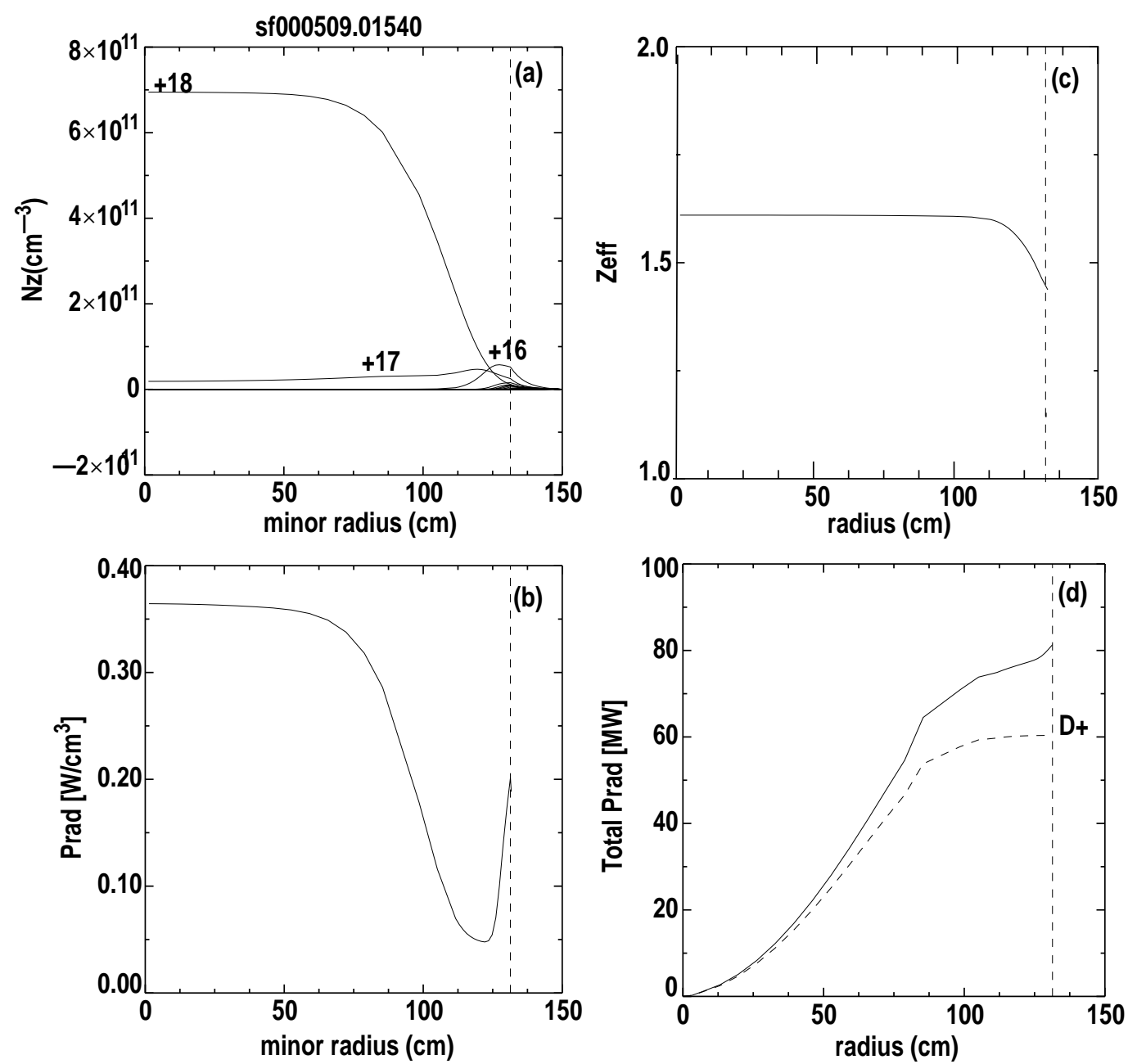

Figure 39: (a) Argon ion density profiles calculated by MIST for $C_{V}=1.0$. The dashed vertical line is at the location of the separatrix.,(b) Argon volume emissivity profile, (c) $Z_{E F F}$, and (d) The radiated power escaping the core plasma. 

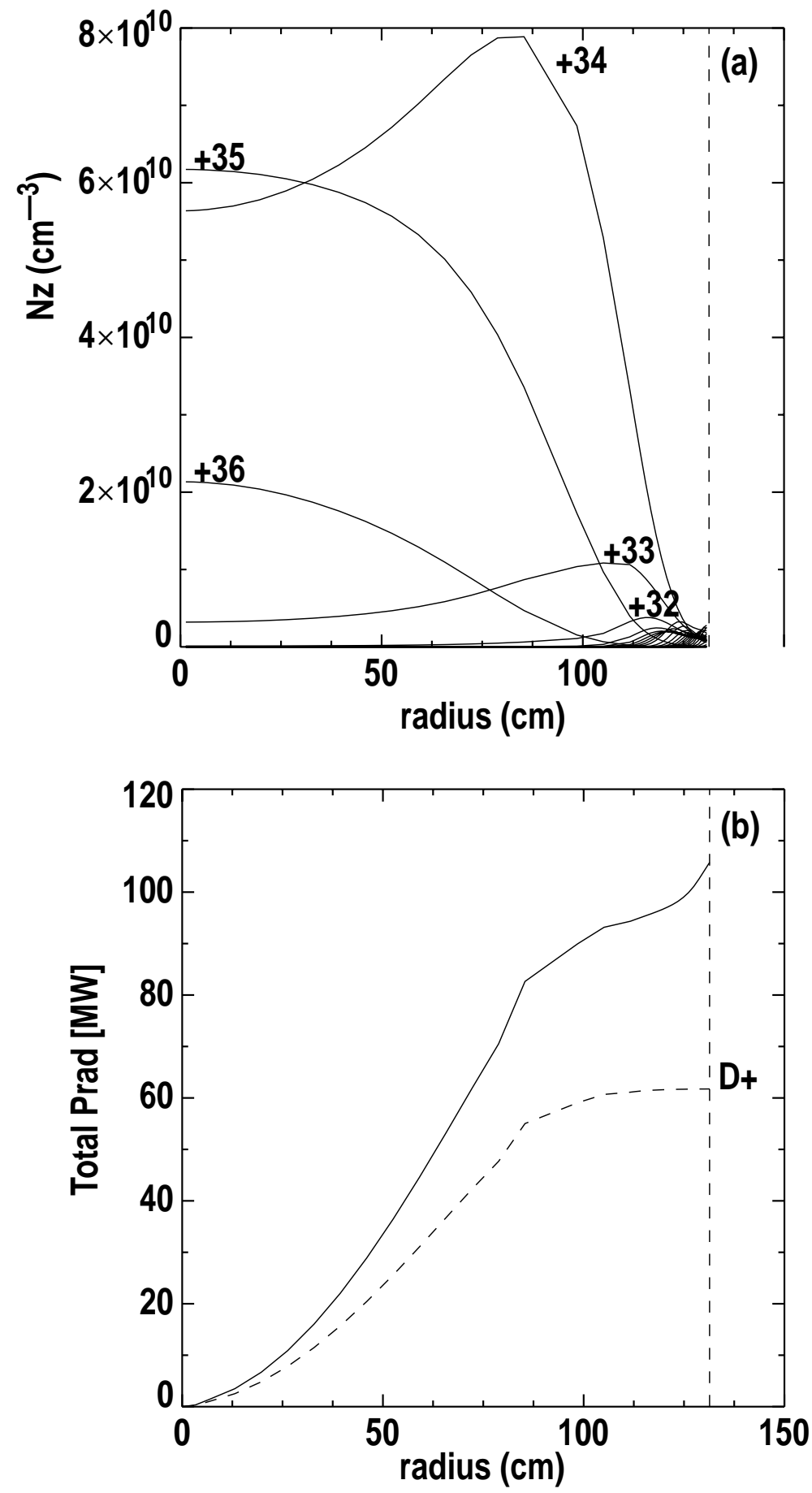

Figure 40: (a) Krypton ion density profiles in ARIES-AT modeled using MIST with $C_{V}=1.0$ and a fixed core fraction of 0.0004 . Krypton is not fully ionized in the core plasma and can produce line radiation; (b) The radiated power escaping the core plasma as a function of minor radius for krypton impurity injection. The solid line is bremsstrahlung and line radiation from krypton, the dashed line is bremsstrahlung from the primary deuterium ions. 
Table 8: Modeling results for rare gas impurity radiation in ARIES-AT: L-mode edge. $P_{\text {rad }}$ includes bremsstrahlung from both the impurity and the fuel ions inside the separatrix. When calculating the radiation fraction, an additional $18 \mathrm{MW}$ of synchrotron radiation is added. The alpha heating plus auxiliary power is assumed to be $360 \mathrm{MW}$. (Note: Core $Z_{\text {eff }}$ does not include the helium content)

\begin{tabular}{|l|l|l|l|l|l|l|}
\hline Impurity & $\mathrm{Z}$ & $C_{V}$ & $\begin{array}{l}\text { Core } \\
\text { Fraction }\end{array}$ & $\begin{array}{l}\text { Core } \\
Z_{\text {eff }}\end{array}$ & $\begin{array}{l}P_{\text {rad }}(M W) \\
\rho<1.0\end{array}$ & $\begin{array}{l}\text { Radiation } \\
\text { Fraction }\end{array}$ \\
\hline Neon & 10 & 1.0 & 0.0075 & 1.7 & 120 & 0.38 \\
\hline Argon & 18 & 1.0 & 0.002 & 1.6 & 140 & 0.44 \\
\hline Argon & 18 & Neo & 0.0023 & 1.7 & 157 & 0.49 \\
\hline Krypton & 36 & 1.0 & 0.0004 & 1.5 & 165 & 0.51 \\
\hline
\end{tabular}

flat density profile and a peaked temperature profile. Near $\rho_{\text {pol }} \approx 0.7$, the density gradient is strong, and the neoclassical pinch is then negative. These transport coefficients result in argon density profiles shown in Figure 42. The peaking of the density near $\rho=0.6$ is expected from the pinch velocity profile shown in Figure 41b. The resulting $Z_{E F F}$ profile is shown in Figure 43.

\subsubsection{Conclusions concerning Impurity Radiation}

The MIST and STRAHL modeling results for impurity radiation induced by injecting a rare gas into the ARIES-AT L-mode edge plasma are summarized in Table 8. With these assumptions the requirements for the overall ARIES-AT system design are met. These results indicate that the higher $\mathrm{Z}$ rare gases are better for the reactor grade plasmas, as they can meet the radiated power requirements at lower $Z_{E F F}$ and lower fuel dilution.

The first wall engineering restriction to keep the core plasma radiation to below 140 MW (or about 36maintaining low total conducted power to the divertor strike plates, implies a need to radiate a large amount of power from the divertor plasma (see Sec. 5.2). These modeling results indicate that with an improved main chamber first wall thermal design, the use of krypton as a core plasma radiator can significantly reduce the divertor radiation requirements.

The dynamics of feedback control of the radiated power using rare gas injection have not been investigated. The large size, and near neoclassical transport levels mean that the impurity confinement times are long. Control of core impurities using the "puff and pump" technique on DIII-D has been demonstrated [62]. Detailed studies of the stability of the burning plasma with feedback control of the rare gas radiated power are needed. 

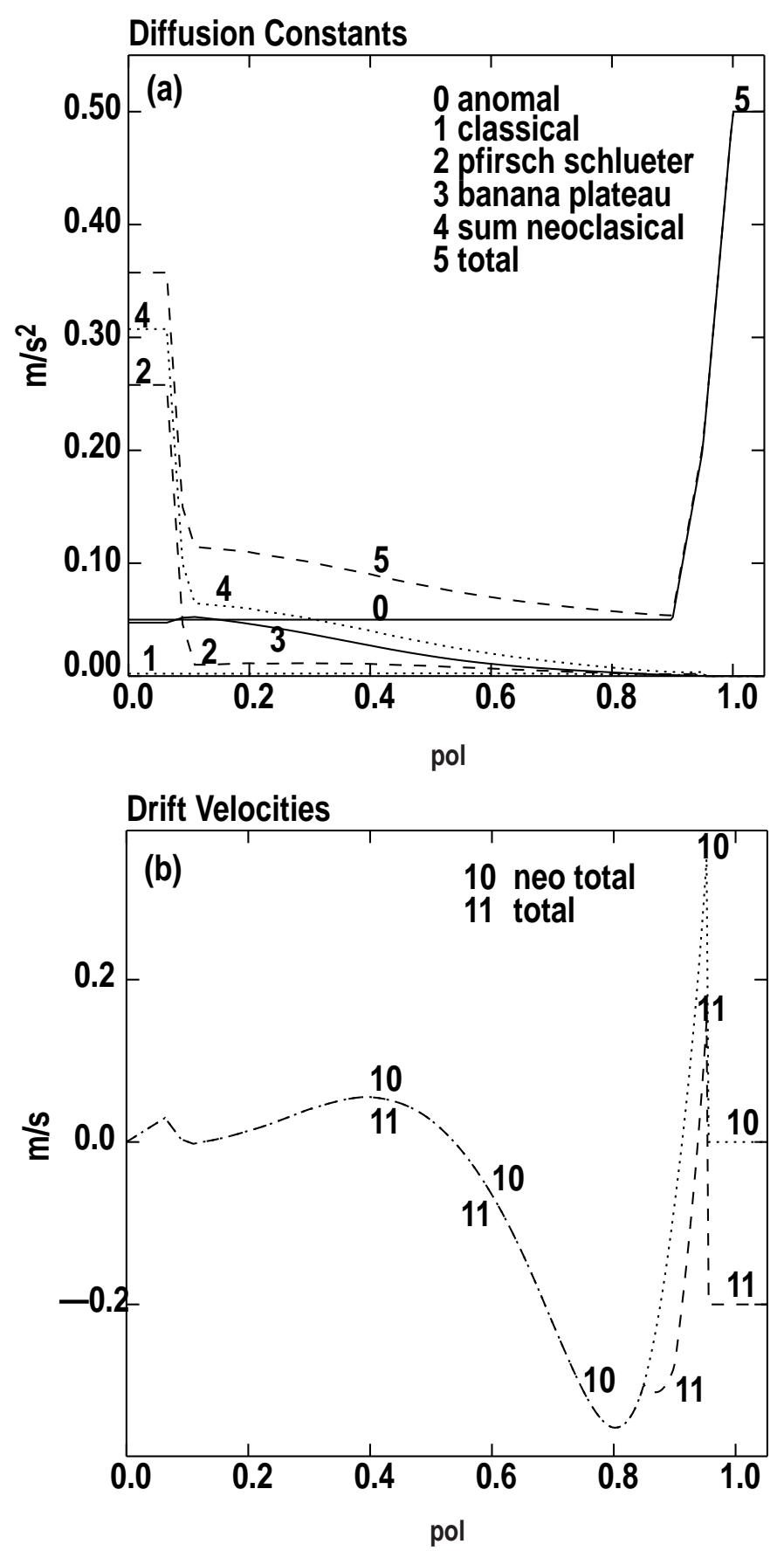

Figure 41: (a) The total diffusion coefficient used in the STRAHL modeling of neoclassical Ar transport in the ARIES-AT L-mode edge plasma is shown as curve 5. A small anomalous term of $0.05 \mathrm{~m}^{2} / \mathrm{s}$ is assumed inside the transport barrier. Outside the transport barrier in the L-mode edge an anomalous contribution of $0.5 \mathrm{~m}^{2} / \mathrm{s}$ is assumed.(b) The profile of the pinch velocity in the neoclassical model of Ar transport in the ARIES-AT l-mode edge plasma. An anomalous pinch of $-0.2 \mathrm{~m} / \mathrm{s}$ is assumed outside of the transport barrier. Inside the transport barrier, no anomalous pinch is used. 


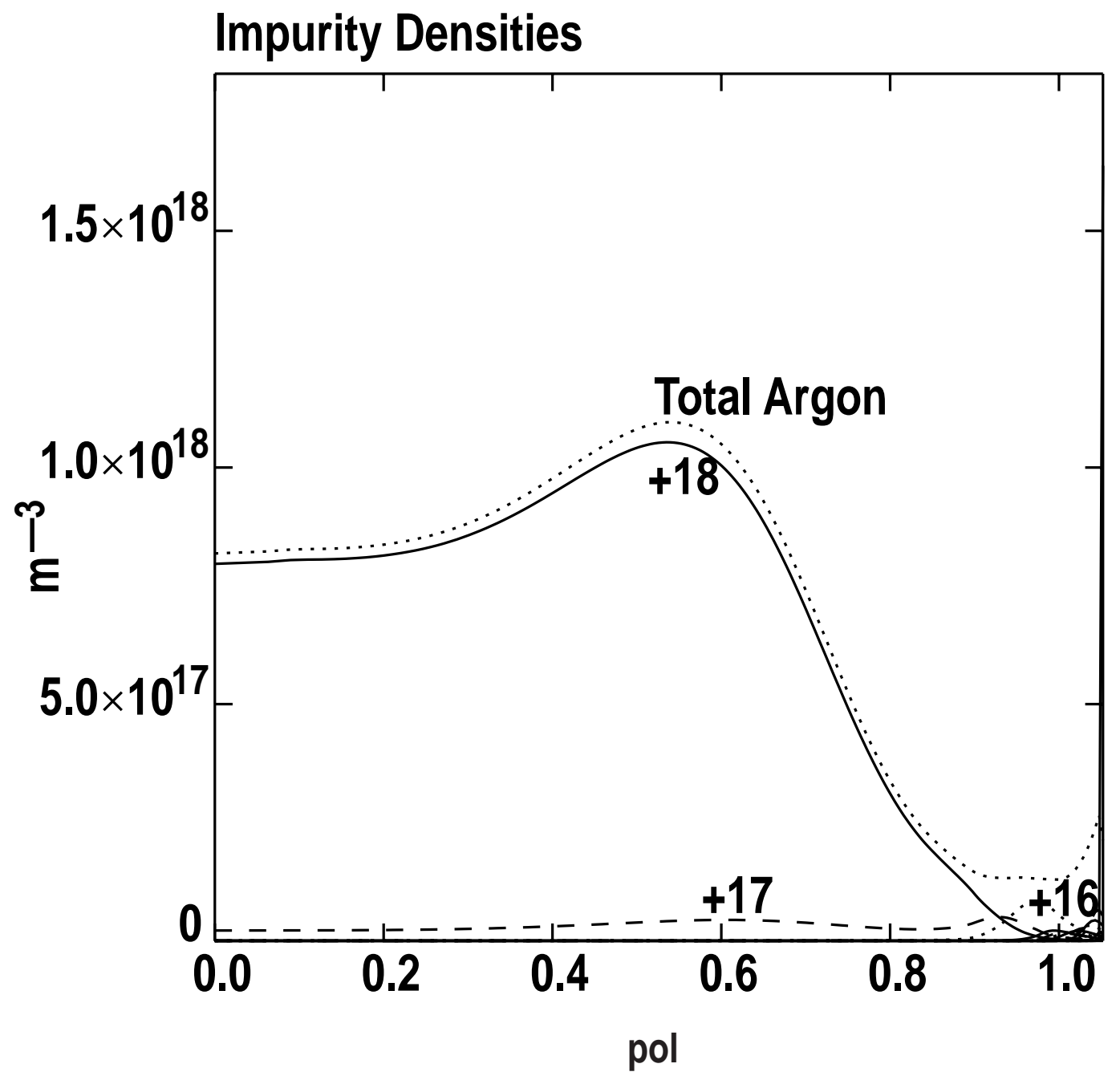

Figure 42: Argon density profiles predicted by the neoclassical model in the STRAHL code. 


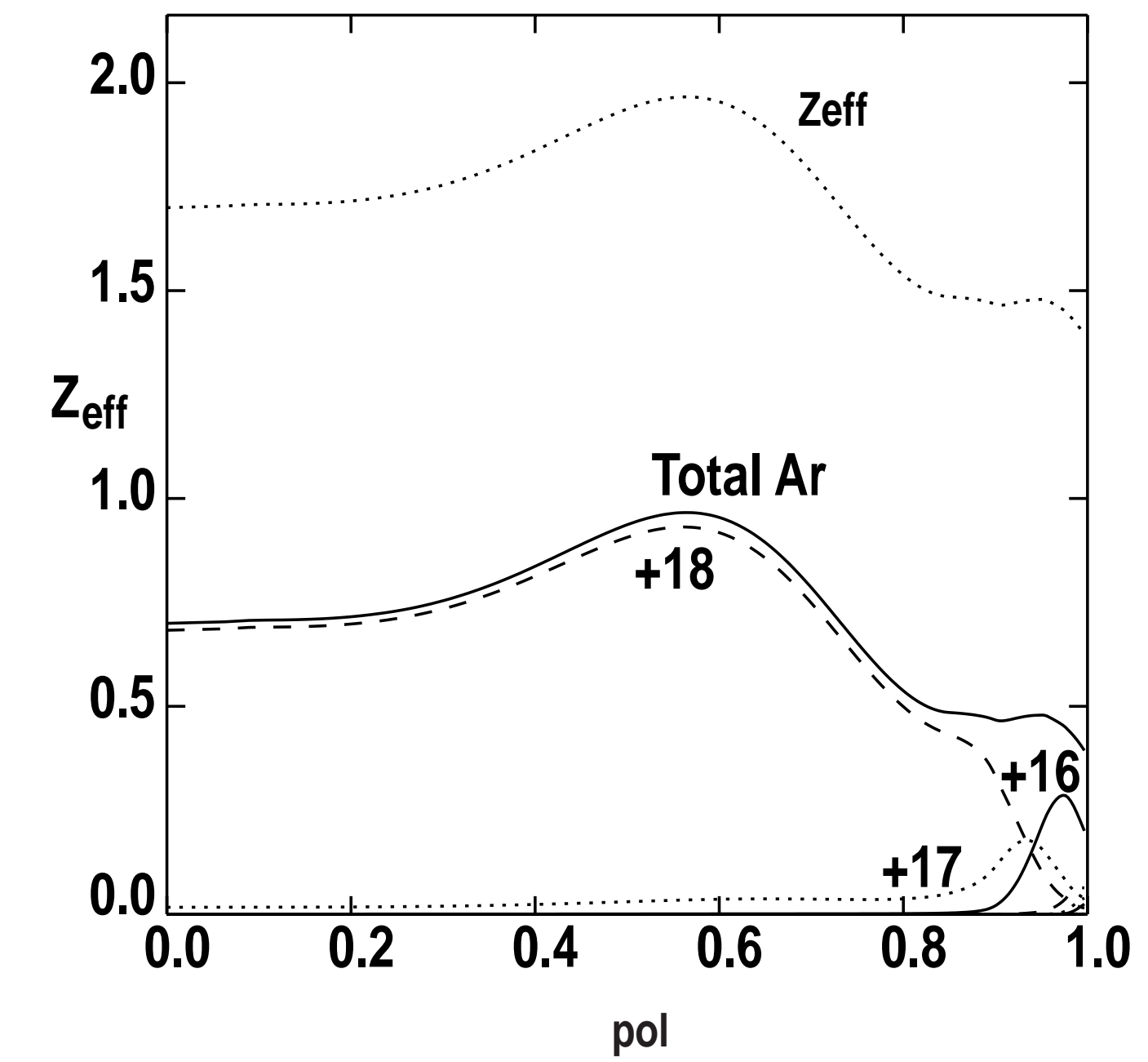

Figure 43: The $Z_{E F F}$ profile from argon predicted by using the neoclassical model in STRAHL for the ARIES-AT L-mode edge. 


\section{Plasma Operating Regime and Startup}

\subsection{POPCON analysis}

A steady-state power balance is enforced to establish the ARIES-AT plasma operating point. Given the input of $P_{C D} \simeq 37 \mathrm{MW}$ of $\mathrm{rf}$ power for current drive (CD), the ARIES-AT plasma operates slightly sub-ignited. At a density-weighted, volumeaverage temperature $T_{i} \simeq T_{e} \simeq 18 \mathrm{keV}$, the Lawson parameter, $n_{i} \tau_{E}$, is $3.42 \times 10^{20}$ $\mathrm{s} / \mathrm{m}^{3}$, as the product of the volume-average ion density, $n_{i}=1.71 \times 10^{20} \mathrm{~m}^{-3}$, and the energy confinement time, $\tau_{E}=2.0 \mathrm{~s}$.

The plasma heating power is $P_{P H}=P_{\alpha}+P_{C D}$, where $P_{\alpha} \simeq 351 \mathrm{MW}$ is the selfheating power from alpha-particle fusion products. These input powers are balanced by transport losses across the plasma separatrix, $P_{T R} \simeq 274 \mathrm{MW}$ and radiation (primarily bremsstrahlung, $P_{B R} \simeq 55 \mathrm{MW}$, line radiation, $P_{L I N E} \simeq 43 \mathrm{MW}$, and cyclotron radiation, $\left.P_{C Y} \simeq 19 \mathrm{MW}\right)$. The core radiation fraction is $f_{R A D} \simeq 0.30$ and $P_{T R}=P_{P H}\left(1-f_{R A D}\right)$.

Once $\tau_{E}$ is known, a comparison can be made to any of a number of empirical scaling relations $[68,69,70]$, with the caveat that the most appropriate relation for the ARIES-AT regime is not yet established experimentally. It is emphasized that a scaling law is not needed to derive any of the ARIES design points; $\tau_{E}$ is determined from the specification of target net electrical power output, $P_{E}$, and plasma gain, $Q_{p}$, that is set by the current-drive power requirement.

Recast in SI units, the L-mode ITER89-P scaling [71, 72, 73] is given by

$$
\tau_{E}^{89 P}=3.8033 \times 10^{-6} I_{p}^{0.85} n_{e}^{0.1} B_{\phi 0}^{0.2} a^{0.3} R_{T}^{1.2} \kappa_{\times}^{0.5} A_{i}^{0.5}\left[P_{T R}\right]^{-0.5}
$$

where $A_{i}$ is the atomic mass (2.5 for a nominal 50:50 D-T fuel mixture). This scaling relation is used here as a representative example.

The value of $\tau_{E}$ required for a design point is compared to the various empirical scaling laws by means of a confinement multiplier, $H^{j} \equiv \tau_{E} / \tau_{E}^{j}$, where the superscript $j$ denotes the particular scaling of interest. The energy-confinement-time multiplier, $H^{j}$, for the ARIES-AT relative to the ITER89-P scaling is $H^{j}=2.65$.

A plasma operating contour (POPCON) [74] plot has been generated for the ARIES-AT using the ITER89-P scaling relation. The results assuming the ITER89$\mathrm{P}$ relation are shown in Fig. 44. The plasma operating space is depicted in terms of density and temperature. The plasma power balance equation is solved, including a term for auxiliary heating power, $P_{A U X}$. Using 10-MW increments, the POPCON plot shows contours of constant values of $P_{A U X}$ with the ignition threshold (i.e., $\left.Q_{p} \rightarrow \infty\right)$ indicated by the first dashed contour. The ignition contour itself shows that the minimum density for ignition is $n_{i} \simeq 1.71 \times 10^{20} \mathrm{~m}^{-3}$ near $T_{i} \simeq 12 \mathrm{keV}$. An ignited plasma to the left of the minimum of this contour is thermally unstable.

The ARIES-AT design point optimization moves to somewhat higher temperature to reduce costs associated with the CD system while reducing the fusion power density a little. The modest $P_{C D}$ input moves the ARIES-AT steady-state operating 
point to the position indicated by the open square. Thermally stable operation near $T_{i} \simeq 18 \mathrm{keV}$ is suggested by this result; other scaling relations might shift this zone.

The product $n T$ is proportional to the plasma beta, $\beta=\beta_{N}\left[I_{p} /\left(a_{p} B_{\phi 0}\right)\right]$. The band defined by $\beta_{N}=5-6$ is depicted by the dash-dot isoquants and the full-up values of $I_{p}, a_{p}$, and $B_{\phi 0}$ for the ARIES-AT. A dotted curve denoting fixed fusion power, $P_{F}=2,000 \mathrm{MW}$ in this $n-T$ space for the ARIES-AT plasma volume is also shown. The fusion power of the ARIES-AT itself is $\sim 1,760 \mathrm{MW}$.

Figure 44 also shows that a start-up transient with increasing plasma density and temperature passing through the saddle region (Cordey pass) near $T_{i} \simeq 7 \mathrm{keV}$ requires auxiliary heating power, $P_{A U X}$, of about $25 \mathrm{MW}$.

\subsection{Plasma Non-inductive Startup}

\subsubsection{Inputs \& Model Equilibrium}

The work described in this section was done using a TD spreadsheet (v. 11.0). This is a 0-D tokamak simulation, which includes calculations of bootstrap current and fusion power based on prescribed density, temperature, and current (q) profiles. Because the bootstrap calculation does not include geometric effects such as the Shafranov shift, the calculated bootstrap current is multiplied by 0.85 in order to match the reference equilibrium. This leads to an underestimate of the bootstrap current at low beta. The external heat sources are from the reference: $33 \mathrm{MW}$ of LH power and $3 \mathrm{MW}$ of FW. The calculated current from these sources is $1.14 \mathrm{MA}$, in agreement with the reference (1.12 MA).

\subsubsection{Rampup Scenario}

With only $36 \mathrm{MW}$ of available external power, the most likely way to make the rampup work is to provide for control of the confinement, allowing H98y $1=2$. Fixed quantities are a, $R, \kappa, \delta, B, q(0), P_{L H}, P_{F W}$, and the normalized profiles of density and temperature. The density is varied with the current at fixed NG. The ramp-up is started arbitrarily at $I_{p}=0.5 M A$. It appears that with LHCD, the current could be started from zero, but the required density variation was not considered.

There are three phases to this rampup. For $0 \leq t \leq 52 \mathrm{~s}$, the plasma is at the assumed $\beta_{p}=4$ limit. During this time, $H_{98 y 1}$ is increased from 0.7 to 2.0. At 52 seconds, $I_{p}=2.6 M A$. For $52 \leq t \leq 886 s, H_{98 y 1}$ is fixed at $2.0, \beta_{p}$ falls below the maximum, and $\beta_{N}$ increases to 5.4 , the maximum allowed. At $886 \mathrm{~s}, I_{p}=7.9 \mathrm{MA}$. Finally, for $886 \leq t \leq 5000 s, \beta_{N}=5.4$, and $H_{98 y 1}$ and $\beta_{p}$ fall to their final values. The total time required to bring the plasma to the operating point is roughly $11 / 2$ hours. 


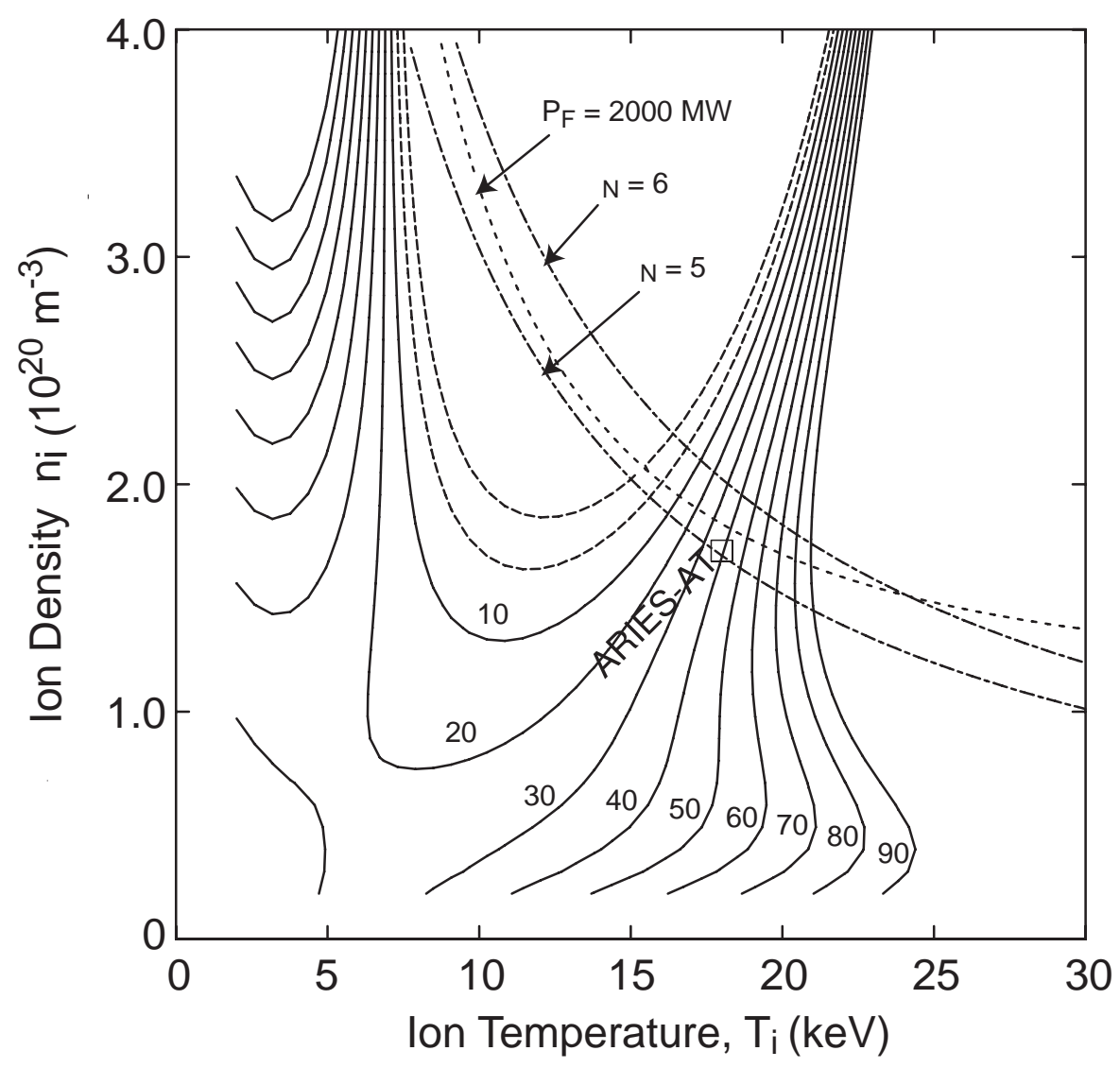

Figure 44: ARIES-AT Plasma Operating Contour (POPCON) diagram. Solid contours indicate auxiliary heating power, $P_{A U X}$, in increments of $10 \mathrm{MW}$ until "ignition" (i.e., $Q_{p} \rightarrow \infty$ ) at the dashed contours. The dash-dot isoquants denote $\beta_{N}=5-6$. The dotted curve traces fixed fusion power, $P_{F}=2,000 \mathrm{MW}$. The steady-state ARIES operating point is denoted by the open square. 


\section{Summary}

ARIES-AT is based on a reversed-shear plasma configuration as was ARIES-RS, but the ARIES-AT $\beta$ is $9.2 \%$, almost twice the $4.96 \%$ value of ARIES-RS. The increased $\beta$ has been used in the design to reduce size of the device somewhat, and to reduce the toroidal field from 8.0 T in ARIES-RS to $5.8 \mathrm{~T}$ in ARIES-AT

The initial optimizations were performed with ideal-MHD analysis of reference equilibrium with zero edge gradients. These equilibrium are typical of L-mode plasmas, but also of H-mode plasmas in the period immediately following an ELM. In addition, explicit analysis of plasma pressure and current profiles typical of H-mode plasmas showed that these profiles, which are characterized by non-zero pressure gradients and current density at the edge, do not reduce the ideal MHD $\beta$ limits. The RWM stability has been analyzed. Calculations show that a stable region for this mode exists in the presence of plasma rotation velocities of $7-10 \% V_{A}$. It was concluded that it is not feasible to maintain these high rotation velocities in a reactor environment, and thus an active feedback system is employed for mode control, requiring about $10 M W$ of power.

The neoclassical tearing mode (NTM) is identified as a problem. Stabilization of this mode by using radially localized ECCD to replace the missing bootstrap current appears to require prohibitively large ECCD power, and future work will concentrate on methods to leverage this by modifying the background current profile (ie, make $\Delta^{\prime}$ more negative).

The analysis of the stability of intermediate mode number ideal MHD modes shows the reference case to be stable to these modes and the H-mode case to be at the marginal stability point. This appears to be consistent with the interpretation of the "reference" (L-mode like) equilibrium to be typical of the post-ELM phase, and the H-mode equilibrium to be typical of the pre-ELM period.

Approximately 91\% of the plasma current in ARIES-AT is self driven by the bootstrap and diamagnetic effects. The required seed current provided by external rf systems is 1.1 MA of LH (36.9 MW) in the outer part of the plasma, and $0.15 \mathrm{MA}$ ICRF (4.7 MW) near the plasma axis. We have concluded that a third rf system targeting the mid-radius region is not required in ARIES-AT as it was in ARIES-RS.

Transport analysis has been carried out to analyze the consistency of the standard profiles in ARIES-AT. It is found that the large Shafranov shift is a strong stabilizing factor. The GLF23 model predicts global energy confinement that exceeds the ARIES-AT design requirements if the density profile is sufficiently peaked. The possibility of increasing the energy confinement through driven sheared rotation from NBI or ICRF was looked at but considered to be not practical for a device with ARIES-AT parameters.

Methods for dispersing the heat loads from ARIES-AT were analyzed. The final design radiates $36 \%$ of the exhaust power (about $140 \mathrm{MW}$ )from the core. Another $40 \%$ of the heating power was radiated in the scrape-off layer (SOL) mostly in the vicinity of the divertor region. 
It is recognized that the ARIES-AT is a very aggressive design. Many of the physics design parameters are outside of those now routinely obtained in tokamaks. However, this is presented as a vision of the "ultimate potential" of a tokamak if the difficulties described in this article can be overcome.

This work supported by USDOE contract AC02-76CH03073.

\section{References}

[1] C. E. Kessel,T. K.Mau, S. C. Jardin, F. Najmabadi, "Plasma Profile and Shape Optimization Studies for Advanced Tokamak Power Plants,ARIES-AT", Fusion Engineering and Design, this issue (2001)

[2] S. C. Jardin, C. E. Kessel, C. G. Bathke, D. E. Ehst, T. K. Mau,F. Najmabadi, T. W. Petrie, "Physics basis for a reversed shear tokamak power plant", Fusion Engineering and Design, 38 (1997) 27

[3] EFIT equilibrium reference number g00509.0*540, General Atomics Company, San Diego, California

[4] M.S. Chu, J.M. Greene, T.H. Jensen, R.L. Miller, A. Bondeson, R.W. Johnson, and M.E. Mauel, Phys. Plasmas, 2, (1995) p. 2236

[5] A. Bondeson,G. Vlad,H. Lutjens, "Resistive toroidal stability of internal kink modes in circular and shaped tokamaks", Physics of Fluids B. Plasma Physics, 4 (1992) 1889

[6] L. C. Bernard,F. J. Helton,R. W. Moore, "GATO: An MHD Stability Code for Axisymmetric Plasmas with Internal Separatrices", Comput. Phys. Commun. 24 (1980) 377

[7] M. S. Chance, "Vacuum calculations in azimuthally symmetric geometry",Physics of Plasmas,4 (1997) 2161

[8] A.H. Boozer, "Feedback equations for the wall modes of a rotating plasma", Phys. Plasmas 6, 3180 (1999).

[9] C. Cates, M. Shilev, M.E. Mauel, G.A. Navratil, D. Maurer, S. Mukherjee, D. Nadle, and A. Boozer, "Supression of resistive wall instabilities with distributed independently controlled active feedback coils", Phys. Plasmas 7, 3133 (2000).

[10] A.M. Garofalo, A.D. Turnbull, E.J. Strait, M. E. Austin, J. Bialek, et al., "Stabilization of the external kink and control of the resistive wall mode in tokamaks", Phys. Plasmas 6, 1893 (1999). 
[11] E. J. Strait, J. Bialek, N. Bogatu, M. Chance, M.S. Chu, D. Edgell, A. M. Garofalo, G. L. Jackson, T.H. Jensen, L.C.Johnson, J.S.Kim, R.J.LaHaye, G.Navratil, M.Okabayashi, H.Reimerdes, J.T.Scoville, A.D. Turnbull, M.L.Walker, and the DIII-D team, Nucl. Fusion, 43,p.430 (2003)

[12] R.J. La Haye, R.J. Buttery, S. Guenter, G.T. A. Huysmans, M. Marascheck, and H.R. Wilson, "Dimensionless scaling of the critical beta for onset of a neoclassical tearing mode", Phys. Plasmas 7, 3349 (2000).

[13] G. Grantenbein, H. Zohm, G. Giruzzi, S. Gnter, F. Leuterer, et al., "Complete suppression of NTM with ECRH CD in ASDEX Upgrade",Phys. Rev. Lett. 85, $1242(2000)$.

[14] A. Pletzer and F.W. Perkins, "Stabilization of Neoclassical tearing modes Using a Continuous Localized Current Drive," Phys. Plasmas 6 (1999) p. 1589

[15] (calculated from code "TEAR," courtesy of T.Gianakon, LANL)

[16] P.B. Snyder, H.R. Wilson, et al, "Edge Localized Modes and the Pedestal: A Model Based on Peeling-Ballooning Modes", Phys. Plasmas 9 (2002) 2037.

[17] J.W. Connor, R.J. Hastie, H.R. Wilson, and R.L. Miller, "Magnetohydrodynamic Stability of Tokamak Edge Plasmas", Phys. Plasmas 5 (1998) 2687.

[18] H.R. Wilson, P.B. Snyder, R.L. Miller, and G.T.A. Huysmans, "Numerical Studies of Edge Localized Instabilities in Tokamaks", Phys. Plasmas 9 (2002) 1277.

[19] C. C. Petty, F. W. Baity, J. S. deGrassie, et al., "Fast Wave Current Drive in H-Mode Plasmas," Nucl. Fusion 39 (1999) 1421.

[20] T. C. Luce, , Y. R. Lin-Liu, J. Lohr, et el., "Generation of Localized NonInductive Current by Electron Cyclotron Waves on the DIII-D Tokamak," Phys. Rev. Lett. 83 (1999) 4550.

[21] M. Thumm, "Recent Development of High Power Gyrotrons and Windows for EC Wave Applications," AIP Conf. Proc. 403 (1997) 183.

[22] T. K. Mau, D. A. Ehst, D. J. Hoffman, "The Radio-Frequency Curent-Drive System for the ARIES-I Tokamak Power Reactor," Fusion Eng. and Design 24 (1994) 205.

[23] T. K. Mau, the ARIES Team, "The Current Drive and Heating Systems for the ARIES-RS Tokamak Power Plant," Proc. 17th IEEE/NPSS Symp. Fusion Eng., Vol. 1 (1997) 425. 
[24] T. K. Mau, S. C. Chiu, R. W. Harvey, "Modelling of Fast Wave Current Drive in Standard and Second-Stability Bootstrapped Reactor Plasmas," Abst. EPS Top. Conf. on Radiofrequency Heating and Current Drive of Fusion Devices, Brussels, Vol. 16E (1992) 181.

[25] Y. Peysson, "Status of Lower Hybrid Current Drive," AIP Conf. Proc. 485 (1999) 183.

[26] S. Itoh, K. N. Sato, K. Nakamura, et al., "Recent Progress on High Performance Steady State Plasmas in the Superconducting Tokamak TRIAM-1M," Nucl. Fusion 39 (1999) 1257.

[27] S. M. Kaye, "Physics Results from the National Spherical Torus Experiment," Bull. Am. Phys. Soc. 45 (2000) 118; paper to be published in Phys. Plasmas (2001).

[28] P. Bonoli, "Linear Theory of Lower Hybrid Heating," MIT Report PFC/RR84-5 (1984).

[29] F. Mirizzi, Ph. Bibet, et al., "Toward an Active Passive Waveguide Array for Lower Hybrid Application on ITER," Proc. 20th Symp. on Fusion Technol. (1998) 465.

[30] C. D. Warrick, M. Valovic, B. Lloyd, et al., "The Confinement and Stability of Quasi-Stationary High- $\beta$ Plasmas on COMPASS-D," 26th EPS Conf. on Contr. Fusion and Plasma Phys. Maastricht (1999) 153.

[31] R. H. Fowler, J. A. Holmes, J. A. Rome, "NFREYA - A Monte Carlo Beam Deposition Code for Noncircular Tokamak Plasmas," Oak Ridge National Laboratory Report ORNL/TM-6845 (1979).

[32] M. Cox, G. Counsell, M. R. O'Brien, et al., "Heating and Current Drive in Tight Aspect Ratio Tokamaks," Abst. 21st EPS Conf. Controlled Fusion and Plasma Phys., Montpellier (1994), vVol. II, p. 1130.

[33] Waltz, R. E., Staebler, G. M., Dorland, W., Hammett, G. W., Kotschenreuther, M., and Konings, J.A., "A gyro-Landau-fluid transport model", Phys. Plasmas 4 (1997) 2482.

[34] Kinsey, J.E., Waltz, R.E., Schissel, D.P., Proc. of the 24th Euro. Conf. on Contr. Fusion and Plasma Phys., Berchtesgaden, Germany (European Physical Society, P-III, 1081) (1997)

[35] Hammett, G. and Perkins, F., "Fluid moment models for Landau Damping with application to the ITG instability", Phys. Rev. Lett. 64 (1990) 3019. 
[36] Kotschenreuther, M., Rewoldt, G., and Tang, W.M., "Comparison of initial value and eigenvalue codes for kinetic toroidal plasma instabilities", Comput. Phys. Commun. 88, 128 (1995)

[37] Beer, M.A., Hammett, G.W., Rewoldt, G., Synakowski, E.J., Zarnstorff, M.C., et al., "Gyrofluid simulations of turbulence suppression in reversed-shear experiments on TFTR", Phys. Plasmas 4 (1997) 1792.

[38] St. John, H., private communication

[39] Kinsey, J., private communication

[40] Waltz, R. E., Kerbel, G. D., Milovich, and J., Hammett, G. W., "Advances in the simulation of toroidal gyro-Landau fluid model turbulence", Phys. Plasmas 22408 (1995).

[41] Stallard, B.W., Greenfield, C.M., Staebler, G.M., Rettig, C.L., Chu, M.S. et al., "Electron heat transport in improved confinement discharges in DIII-D", Phys. Plasmas 6 (1999) 1978.

[42] Jackson,G.L., Winter, J., Taylor, T.S., Burrell, K.H., DeBoo, J.C., et al., "Regime of very high confinement in the Boronized DIII-D tokamak", Phys. Rev. Lett. 67 (1991) 3098.

[43] Koide Y., et al. , "Internal transport barrier with improved confinement in the JT-60U tokamak", Plasma Phys. Control. Fusion 38 (1996) 1011.

[44] Rice, J.E., et al "Observations of Toroidal Rotation Suppression with ITB Formation in ICRF and Ohmic H-mode Alcator C-Mod Plasmas" IAEA Fusion Conference, Sorrento, Italy, October, 2000.

[45] Perkins, F.W., et al "Generation of Plasma Rotation in a Tokamak by IonCyclotron Absorption of Fast Alfven Waves" IAEA Fusion Conference, Sorrento, Italy, October, 2000.

[46] A. Loarte, et. al., "Multi-machine scaling of the divertor peak heat flux and width for L-mode and H-mode discharges", J. Nucl. Mater., 266-269 (1999) 587.

[47] V. Riccardo, W. Fundamenski, G.F.Matthews, Plasma PHys. Control. Fusion 43 (2001) 881.

[48] T.W. Petrie, et. al., "The Effect of Divertor Magnetic Balance on H-mode performance in DIII-D", Presented at the 14th International Conference on PlasmaSurface Interactions in Controlled Fusion Devices, Rosenheim, Germany, May 21-26, 2000 (to be published in J. Nucl. Mater.). 
[49] T. W. Petrie, C.M.Greenfield, R.J. Groebner, A.W. Hyatt, R.J.Lahaye, et al., J. Nucl. Mater. 290-293 (2001) 935.

[50] T.W. Petrie, M.E. Fenstermacher, C.J. Lasnier, Fusion Technol. 39 (2001) 916.

[51] A.M. Messiaen, J. Ongena, U. Samm, B. Unterberg, G. Van Wasserhove, et al. Phys. Rev. Lett 77 (1996) 2487

[52] K.W. Hill, S. D. Scott, M. Bell, R. BUdny, C.E. BUsh, et al., Phys. Plasmas 6 (1999) 877

[53] G. L. Jackson, M. Murakami, G.M. Staebler, M.R. Wade, A.M. Messiaen, et al., J. Nucl. Mater. 266-269 (1999) 380.

[54] G. L. Jackson, M. Murakami, G.R. Mckee, D.R. Baker, J.A. Boedo, et al., Nucl. Fusion 42 (2001) 28.

[55] G. F. Matthews, B. Balet, J.G. Cordey, S.J. Davies, G.M. Fishpoll, et al., Nucl. Fusion 39 (1999) 19.

[56] J. Ongena, W. Suttrop, M. Becoulet, G. Cordey, P. Dumortier, et al., Plasma PHys. and Contr. Fusion 43 (2001) A11.

[57] H. Kubo, S. Sakurai, N. Asakura, S. Konoshima, H. Tamai, et al., Nucl. Fusion 41 (2001) 227.

[58] T.D. Rognlien, et. al., "Influence of ExB and $\nabla B$ drift terms in 2D edge/SOL transport simulations", J. Nucl. Mater. 266-269 (1999) 654.

[59] J.A. Boedo, et al., "Electric field-induced plasma convection in tokamak divertors", Phys. Plasmas 7 (2000) 1075.

[60] T. D. Rognlien, P. N. Brown, R. B. Campbell, et. al., Contrib.Plasma Phys. 34 (1994) 362.

[61] M. Rensink and T. Rognlien (private communication, 2000).

[62] M.R. Wade, et al., "Impurity enrichment studies with induced scrape-off layer flow on DIII-D", Nucl. Fusion, 38 (1998) 1839.

[63] F. W. Perkins, et. al, "ITER Physics Basis", Nucl. Fusion 39 (1999) 2391.

[64] R.A. Hulse, Nuclear Technology/Fusion "Numerical studies of impurities in fusion plasmas",3, 259 (1983)

[65] K. Behringer, "Description of the impurity transport code STRAHL", JETR(87)08, JET Joint Undertaking, Culham (1987) 
[66] A.G. Peeters, "Reduced Charge State Equations that describe Pfirsch-Schluter impurity transport in tokamak plasma", Physics of Plasmas (1999)

[67] M.R. Wade, W.A.Houlberg, L.R.Baylor, " Experimental Confirmation of Impurity Convection Driven by the Ion Temperature Gradient in Toroidal Plasmas", Phys. Rev. Lett., 84282 (2000)

[68] "ITER Conceptual Design Interim Report,"ITER Documentation Internal Series No. 7, International Atomic Energy Agency, Vienna (1989).

[69] N. A. Uckan, "Tokamak Confinement Projections and Performance Goals," Fusion Technol. 15 (March 1989) 391.

[70] F. W.Perkins, et. al. "ITER Physics Basis," Nuclear Fusion, 39, 12 (1999) Chapter 2, Table 5. p. 2208.

[71] P. N. Yushmanov, T. Takizuka, K. S. Riedel, O. J. W. F. Karduan, J. G. Cordey, S. M. Kaye, and D. E. Post, "Scalings for Tokamak Energy Confinement,"

[72] D. E. Post, K. Borrass, J. D. Callen, S. A. Cohen, J. G. Cordey, F. Engelmann, et al., "ITER Physics," International Thermonuclear Experimental Reactor documentation series No. 21 (November 1990).

[73] S. M. Kaye, et al., "ITER L Mode Confinement Database," Nuclear Fusion, 37, 9 (1997) 1303.

[74] W. A. Houlberg, S. E. Attenberger, and L. M. Hively, "Contour Analysis of Fusion Reactor Plasma Performance," Nuclear Fusion, 22, 7 (1982) 935. 


\section{External Distribution}

Plasma Research Laboratory, Australian National University, Australia

Professor I.R. Jones, Flinders University, Australia

Professor João Canalle, Instituto de Fisica DEQ/IF - UERJ, Brazil

Mr. Gerson O. Ludwig, Instituto Nacional de Pesquisas, Brazil

Dr. P.H. Sakanaka, Instituto Fisica, Brazil

The Librarian, Culham Laboratory, England

Mrs. S.A. Hutchinson, JET Library, England

Professor M.N. Bussac, Ecole Polytechnique, France

Librarian, Max-Planck-Institut für Plasmaphysik, Germany

Jolan Moldvai, Reports Library, Hungarian Academy of Sciences, Central Research Institute for Physics, Hungary

Dr. P. Kaw, Institute for Plasma Research, India

Ms. P.J. Pathak, Librarian, Institute for Plasma Research, India

Ms. Clelia De Palo, Associazione EURATOM-ENEA, Italy

Dr. G. Grosso, Instituto di Fisica del Plasma, Italy

Librarian, Naka Fusion Research Establishment, JAERI, Japan

Library, Laboratory for Complex Energy Processes, Institute for Advanced Study, Kyoto University, Japan

Research Information Center, National Institute for Fusion Science, Japan

Dr. O. Mitarai, Kyushu Tokai University, Japan

Dr. Jiangang Li, Institute of Plasma Physics, Chinese Academy of Sciences, People's Republic of China

Professor Yuping Huo, School of Physical Science and Technology, People's Republic of China

Library, Academia Sinica, Institute of Plasma Physics, People's Republic of China

Librarian, Institute of Physics, Chinese Academy of Sciences, People's Republic of China

Dr. S. Mirnov, TRINITI, Troitsk, Russian Federation, Russia

Dr. V.S. Strelkov, Kurchatov Institute, Russian Federation, Russia

Professor Peter Lukac, Katedra Fyziky Plazmy MFF UK, Mlynska dolina F-2, Komenskeho Univerzita, SK-842 15 Bratislava, Slovakia

Dr. G.S. Lee, Korea Basic Science Institute, South Korea

Institute for Plasma Research, University of Maryland, USA

Librarian, Fusion Energy Division, Oak Ridge National Laboratory, USA

Librarian, Institute of Fusion Studies, University of Texas, USA

Librarian, Magnetic Fusion Program, Lawrence Livermore National Laboratory, USA

Library, General Atomics, USA

Plasma Physics Group, Fusion Energy Research Program, University of California at San Diego, USA

Plasma Physics Library, Columbia University, USA

Alkesh Punjabi, Center for Fusion Research and Training, Hampton University, USA

Dr. W.M. Stacey, Fusion Research Center, Georgia Institute of Technology, USA

Dr. John Willis, U.S. Department of Energy, Office of Fusion Energy Sciences, USA

Mr. Paul H. Wright, Indianapolis, Indiana, USA 
The Princeton Plasma Physics Laboratory is operated by Princeton University under contract with the U.S. Department of Energy.

\author{
Information Services \\ Princeton Plasma Physics Laboratory \\ P.O. Box 451 \\ Princeton, NJ 08543
}

Phone: 609-243-2750

Fax: 609-243-2751

e-mail: pppl_info@pppl.gov

Internet Address: http://www.pppl.gov 